\title{
Soil Contamination Standards for Protection of Personnel
}

Prepared for the U.S. Department of Energy 


\section{Soil Contamination Standards for Protection of Personnel}

Date Published

May 1997

Prepared for the U.S. Department of Energy

Fluor Daniel Hanford, Inc.

P.O. Box 1000

Richland, Washington 


\section{INFORMATION CLEARANCE FORM}

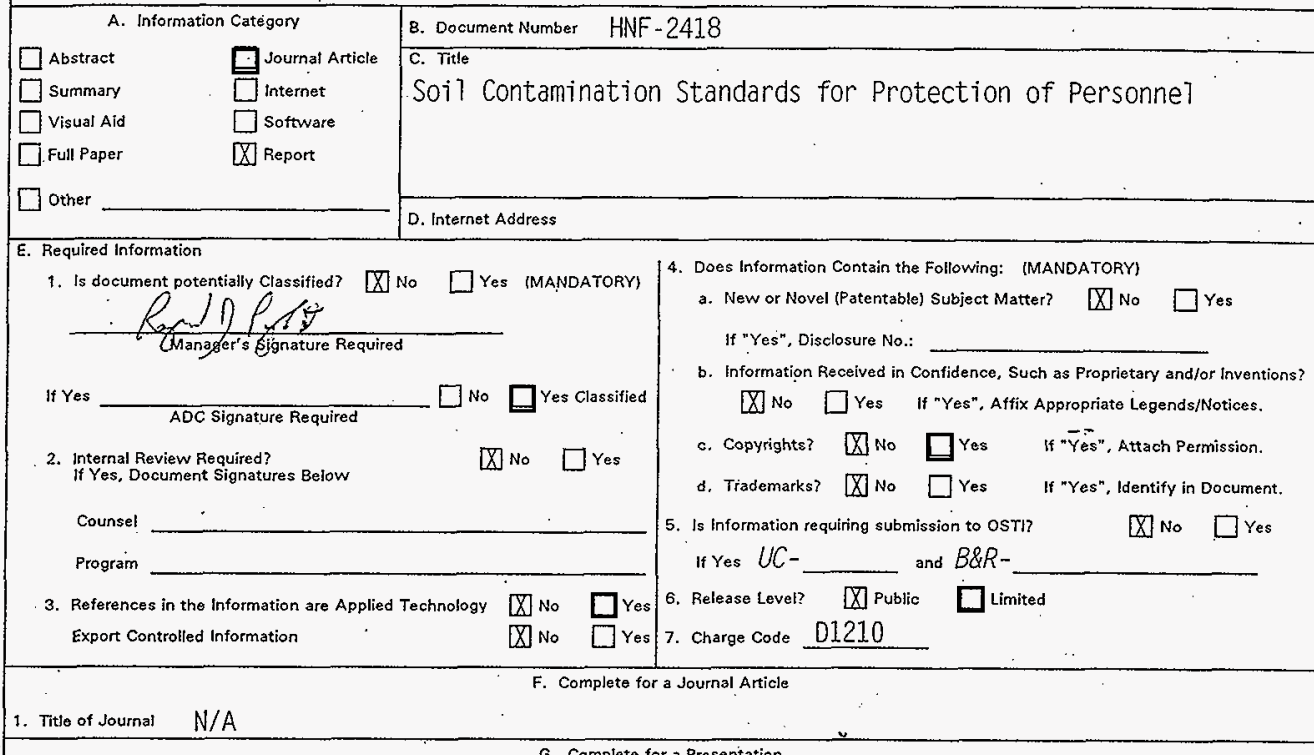

G. Complete for a Presentation

1. Title for Conference or Meeting $\mathrm{N} / \mathrm{A}$

2. Group Sponsoring

3. Date of Conference

5. Will Information be Published in Proceedings? $\square$ No $\square$ Yes

4. City/State

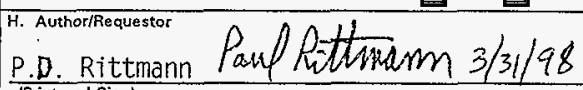

6. Will Material be Handed Out? $\square$ No $\square$ Yes

Responsible Manatger

(Print and sign)

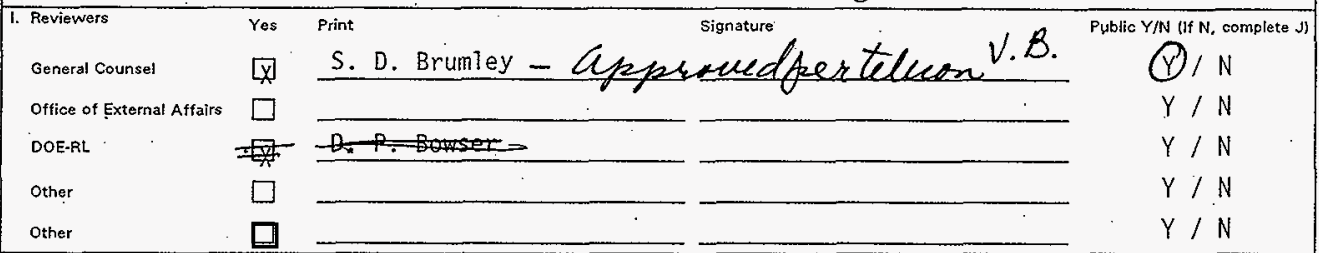

J. If Information Includes Sensitive Information and is not to be released to the Public indicate category below.
$\square$ Applied Technology
$\square$ Personal/Private
$\square$ Exotected CRADA
$\square$ Proprietary
$\square$ Business-Sensitive
$\square$ Predecisional
$\square$ Procurement-Sensitive

$\square$ ucrs

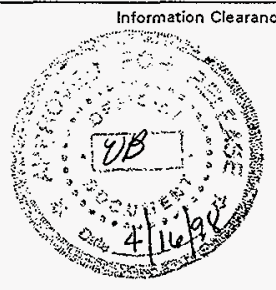




\section{RELEASE AUTHORIZATION}

Document Number: HNF-2418

So 11 Contamination Standards

for Protection of Personnel

This document, reviewed in accordance with DOE Order 1430.1D, "Scientific and Technical Information Management," and DOE G 1430.1D-1, "Guide to the Management of Scientific and Technical Information," does not contain classified or sensitive unclassified information and is:

\section{APPROVED FOR PUBLIC RELEASE}

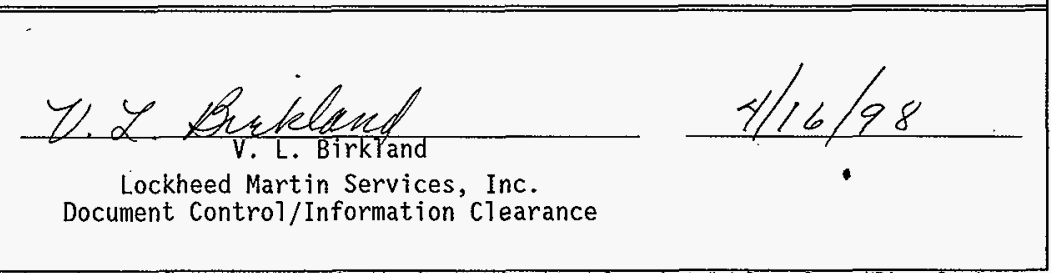

Feviewed for Applied Technology, Business Sensitive, Classified, Copyrighted, Export Controled, Patent, PersonallPrivate, Proprietary, Protected CRADA, Trademark, Unclassified Controlled Nuclear Information.

Trademark Disclaimer. Reforence herein to any specific commercial product, process, or service by trade name, trademark, manufacturer, or otherwise, does not necessarily constitute or imply its endorsement, recommendation, or favoring by the United States Government or any agency thereof or its contractors or subcontractors. The views and opinions of authors expressed herein do not necessarily state or reflect those of the United States Government or any agency thereot. This report has been reproduced from the best available copy.

Printed in the United States of America.

Available to the U.S. Department of Energy and its contractors from the U.S. Department of Energy Office of Scientific and Technical information, P.O. Box 62, Oak Ridge, TN 37831; Telephone: 423/576-8401.

Available to the public from the U.S. Department of Commerce National Technical Information Service, 5285 Port Royal Road, Springfield, VA 22161; Telephone: 703/487-4650. 


\section{CONTENTS}

1.0 Introduction ...................... 1

2.0 Summary of Methods .................. I

3.0 Summary of Results. . . . . . . . . . . . . 2

4.0 Treatment of Mixtures ............... 3

5.0 List of References . . . . . . . . . . . . 17

Appendix A. Data and Formulas Used in the Calculations . . . . . . 18

Appendix B. Method for Estimating Field Instrument Readings . . . . . 29

Appendix C. The DETECT Program Version 2.0.......... 37

\section{TABLES}

Table 1. Unit Dose Factors and Soil Concentration Limits . . . . . . 5

Table 2. Soij Limits and Instrument Response -1 mrem/y ....... 8

Table 3. Soi1 Limits and Instrument Response - $100 \mathrm{mrem} / \mathrm{y} \ldots \ldots . . . .11$

Table 4. Soil Limits and Instrument Response - $500 \mathrm{mrem} / \mathrm{y}$...... 14

Table A-1. Decay Chains Computed in the Spreadsheet . . . . . . . . . 21

Table A-2. Composition of the "+D" Nuclides .......... 22

Table A-3. Solubility Assumptions; Half Lives and Dose Factors . . . . 23

Table A-4. Assumed Composition of Plutonium $6 \%$ Mixture . . . . . . 28

Table B-1. Dose Rates 1 meter Above Various Circular Areas . . . . . . 30

Table B-2. R/rem Ratios and Detection Fractions ........ 31

Table $\dot{C}-1$. Files Distributed as the DETECT Software ....... 38

Table $\mathrm{C}-2$. Help Screen for DETECT .................. 39

Table $\mathrm{C}-3$. Contents of TEST.NUC with Line Numbers Added ...... 40

Table C-4. Output from TEST.NUC Using the Program Defaults . . . . . 41

Table C-5. Range in Air for Various Alpha and Electron Energies . . . 43 
Soil Contamination Standards for Protection of Personnel

by PauT D. Rittmann, PhD CHP May 14, 1997

\subsection{Introduction}

The objective of this report is to recommend soil contamination levels that will ensure that radionuclide intakes by unprotected workers are likeiy to give internal doses below selected dose limits during the working year. The three internal dose 7 imits are 1, 100, and 500 mrem per year. In addition, photon, beta, and alpha instrument readings are estimated for these soil concentration limits.

Two exposure pathways are considered: the first is inhalation of resuspended dust and the second is ingestion of trace amounts of soil. In addition, radioactive decay and ingrowth of progeny during the year of exposure-is included. External dose from the soil contamination is not included because monitoring and control of external exposures is carried out independently from internal exposures, which are the focus of this report. The methods used are similar to those used by Carbaugh and Bihl (1993) to set bioassay criteria for such workers.

\section{0 summary of Methods}

The inhalation dose from resuspended dust is calculated using a mass loading approach. The air breathed by the worker is assumed to have an average dust loading of $5 \mathrm{mg} / \mathrm{m}^{3}$ of respirable sized particles, i.e., less than $10 \mu \mathrm{m}$ AMAD. The worker is assumed to breathe this dust for 2000 hours at the light activity breathing rate for reference man (ICRP 1975), $1.2 \mathrm{~m}^{3}$ /hour. The total amount of dust inhaled is therefore 12 grams over the course of the year. It is assumed that the radionuclide concentration in the airborne dust is the same as it is in the contaminated soil. Technically, this is incorrect. The soil contamination is normally found in the soil fines so that the radionuclide concentration in the airborne dust is far greater than in the bulk soi7. However, the assumed average air concentration of $5 \mathrm{mg} / \mathrm{m}^{3}$ is also very large. It is the OSHA limit for workplace dust. In addition, the assumed exposure time of 2000 hours per year at this high concentration also leads to large intakes. Therefore, it will be assumed that the above combination of assumptions represent a reasonable worst case inhalation model.

The ingestion dose from eating trace amounts of soil is based on the EPA soil ingestion value of $100 \mathrm{mg} /$ day for adults. The ingestion takes place through licking the lips and similar motions. The worker is assumed to consume soit at this rate for 250 days during the year. The total amount ingested in a year is therefore 25 grams of soil.

Appendix A summarizes the data used in these calculations. The 128 distinct radionuclides have half-lives greater than 33 days. Implicit daughters shown in Table A-2 have been included in the calculations of dose. It is assumed that these short-lived nuclides are in secular equilibrium with the parent at a11 times. The radioactive decay information is from Kocher (1981). This was used to determine the relative amounts of the short-lived progeny, as well as the decay and ingrowth of Ionger-lived progeny during the year of exposure. The specific decay chains used during the year of exposure are listed in Table A-1. 
The internal dose factors used in the dose calculations are from EPA (1988). These dose factors are use the methods of ICRP 26 (1977) and are based on a 50 year dose commitment period. Organ doses are not considered in this report. Only the weighted sum of the organ doses, known as the effective dose equivalent is used. Table A-3 lists the inhalation and ingestion dose factors along with the lung solubility class and gut absorption factors which were assumed. The inhalation dose factors use $1 \mu \mathrm{m}$ AMAD particles. The worst-case dose factors were used, except for strontium and uranium. For both eTements, the class $Y$ compounds give the largest doses per unit intake. However, these class $Y$ compounds have never been handled or produced in the 200 Areas. For the 300 Areas, there has been some class $Y$ uranium handled. This was treated separately, for comparison.

The external dose rate factors used in the dose calculations are from EPA (1993). These dose factors are use the weighting factors from ICRP 26 (1977). Table A-3 lists the external dose rate factors for an infinitely thick layer of contaminated soil at the surface. The soil density is $1.6 \mathrm{~g} / \mathrm{cc}$. -..

The method for estimating instrument response is described in Appendix $B$. This method assumes the soil contamination is very uniformly mixed and is at the surface. Due to the non-uniform nature of soil, this is never the exactly the case. For higher energy beta-emitters, the assumed homogenous distribution of contamination in the surface soil introduces little error, except in rocky soils. However, readings from alpha-emitters and low energy beta-emitters are strongly affected by non-uniformities in the distribution of contamination in the surface soil. The estimated readings for these nuclides could be high by orders of magnitude. The users of these soil contamination standards must take into account this weakness on the readings given for alpha and low-energy beta emitting nuclides.

The minimum $C P$ reading is assumed to be $1 \mathrm{mR} / \mathrm{h}$, window closed. Window open readings were not estimated because the GM would give a more sensitive indication of contamination. The minimum GM reading is assumed to be $100 \mathrm{cpm}$ with a $15 \mathrm{~cm}^{2}$ probe face. The PAM has a face area of $50 \mathrm{~cm}^{2}$. The minimum PAM reading for a 2 inch per second scan rate is assumed to be $35 \mathrm{cpm}$. If the PAM registers anything, it is assumed that a static count will be done, which offers a minimum PAM reading of $6 \mathrm{cpm}$. The $6 \mathrm{cpm}$ value is used in the summary tables for the PAM readings. Both the GM and the PAM are assumed to be held about $1 \mathrm{~cm}$ above the soil surface during the scan.

\subsection{Summary of Results}

Table 1 shows the effective dose equivalent (EDE) the worker could receive in the inhalation and ingestion scenario described above. The 50 year dose commitments shown on the table accumulate over the course of one working year. Next to the column with doses is a column showing whether the inhalation or ingestion pathway gives most of the total dose.

Nuclides which have implicit daughters are identified with the letters " $+D "$ after the name. For these nuclides, the activity in the table is only that of the parent nuclide. For example, I pCi/g of $5 \mathrm{r}-90+0$ will also have $\mathrm{I} \mathrm{pCi/g}$ of Y-90. The implicit daughters represent additional activity.

The last three columns of Table 1 give soil concentration limits for individual nuclides based on the three annual dose 1 imits, 1, 100, or 500 mrem. 
Tables 2, 3, and 4 repeat the soil concentration limits shown on Table 1 and also give the estimated instrument reading at the limit. The instruments considered are the window-closed $C P$, the GM with a pancake probe (15 $\mathrm{cm}^{2}$ face area), and the Portable A7pha Monitor (PAM) (50 $\mathrm{cm}^{2}$ face area). It was assumed that the detection limits are $1 \mathrm{mR} / \mathrm{h}$ for the $\mathrm{CP}$, $100 \mathrm{cpm}$ for the GM, and $6 \mathrm{cpm}$ for the PAM. The detection efficiencies depend on the energy of the radiation emitted. Appendix $B$ describes the method used to estimate detector response.

For both the GM and the PAM, the detection limit as well as the detection efficiency depend strongly on the condition of the soil. For example, soil moisture reduces the ability of an instrument to detect alpha particles and low-energy electrons. In addition, the granular nature of soil could lead to readings which are much higher or lower, depending on whether the contamination lies above or below the soil grains.

The last column of Tables 2, 3, and 4 shows which instruments (CP, GM; or PAM) would detect the radionuclide at the concentration given in the second column. For nuclides which undergo significant decay during the year, the concentration shown is at the beginning of the work year. The number after the instrument identifier is the order of magnitude above the assumed detection limit. Thus, "CPO" or "GMO" or "PAMO" aT1 mean that the estimated instrument reading is somewhere between the minimum detectable and 10 times the minimum detectable. Similarly, "CP1" or "GMI" or "PAMI" al1 mean that the estimated instrument reading is somewhere between 10 and 100 times the minimum detectable. In some cases the estimated reading would be off scale, but no notation to this effect is made, since the focus here is on detecting the suggested soil contamination standard.

Nearly all of the beta-emitting nuclides are detectable with field instruments at the $100 \mathrm{mrem} / \mathrm{y}$ level. The few nuclides that are not detectable are normally found with nuclides that can be readily detected. Note that the alpha-emitting nuclides are largely undetectable at the soil contamination limits derived here.

Note also that the instrument readings will give little indication of the depth of contamination. All of the beta particles will come from the upper 4 centimeters of soi1. Most of the photon reading (greater than 80 percent) is from the upper 15 centimeters. These thicknesses of soil are also adequate to conceal subsurface contamination. When soil is being excavated, the radiological conditions at the work site can change rapidly as contamination is uncovered.

\subsection{Treatment of Mixtures}

After a few months or years, some nuclides shown on the table will be in secular equilibrium with longer-lived progeny. Two examples are the $\mathrm{Zr}-95$ to $\mathrm{Nb}-95$ chain and the Sb-125 to Te-125m chain. With the chains in equilibrium they could be combined much the way the short-lived implicit daughters were. These combinations are listed in the last several lines at the end of the tabies. The speciat identifier "old" is used for these chains. As before, the activity concentration shown is only the activity of the parent nuclide. For example, $1 \mathrm{pC} i / g$ of " $\mathrm{Zr}-95$ old" means the concentration of the $\mathrm{Zr}-95$ is $1 \mathrm{pCi} / \mathrm{g}$. There is also the equilibrium amount of $2.21 \mathrm{pCi} / \mathrm{g}$ of $\mathrm{Nb}-95$. As with the implicit daughters, the activity of the progeny in equilibrium is not shown. 
The mixture "Pb-210 old" includes both the short-1ived $\mathrm{Bi}-210$ as well as Po-210. The "Ra-226 old" includes every nuclide down to Po-210 in secular equilibrium. The radionuclide mixtures "Th-Nat" and "U-Nat" are for the complete decay chains of U-238 and Th-232 in secular equilibrium. This is approximately how they are found in nature. Note that background soil concentrations normally range from 0.5 to $1 \mathrm{pCi} / \mathrm{g}$.

Plutonium is normally found as a mixture of isotopes. A typical mixture at Hanford has about 6 percent Pu-240 by mass. This is the mixture labelled "Pu 6\%" at the end of the tables. This mixture was also aged 10 years and recomputed. This is was labelled "Pu 6\% Aged" on the tables. The results differ only slightly from the fresh plutonium.

other mixtures of radionuclides can be evaluated by summing the products of the soil concentrations and the unit concentration dose factor in the first column of Table 1. For example, a mixture of $5,000 \mathrm{pCi} / \mathrm{g} \mathrm{Sr}-90$ and $15,000 \mathrm{pCi} / \mathrm{g} \mathrm{Cs}-137$ would produce the annual dose shown below. Note-that the $E D E$ is given in mrem/y to indicate that the intakes occur over one working year. The dose commitment period is still 50 years. The units are thus the dose commitment per year of exposure.

$\mathrm{Sr} \& \mathrm{Cs}$ Mixture $=(5000 \mathrm{pCi} / \mathrm{g})(0.00672 \mathrm{mrem} / \mathrm{y} \mathrm{per} \mathrm{pCi} / \mathrm{g})+$ $(15000 \mathrm{pCi} / \mathrm{g})(0.00161 \mathrm{mrem} / \mathrm{y}$ per $\mathrm{pCi} / \mathrm{g})$

$=33.6+24.2 \mathrm{mrem} / \mathrm{y}$

$=57.8 \mathrm{mrem} / \mathrm{y}$ 
Table 1. Unit Dose Factors and Soil Concentration Limits

\begin{tabular}{|c|c|c|c|c|c|}
\hline Nuclide & $\begin{array}{l}\text { TEDE } \mathrm{mrem} / \mathrm{y} \\
\text { at } 1 \mathrm{pCi} / \mathrm{g}\end{array}$ & $\begin{array}{l}\text { Max } \\
\text { Pathway }\end{array}$ & $\begin{array}{l}\mathrm{pCi} / \mathrm{g} \text { to } \\
1 \text { mrem }\end{array}$ & $\begin{array}{l}\text { live the an } \\
100 \text { mrem }\end{array}$ & $\begin{array}{l}1 \text { al doses } \\
500 \text { mrem }\end{array}$ \\
\hline 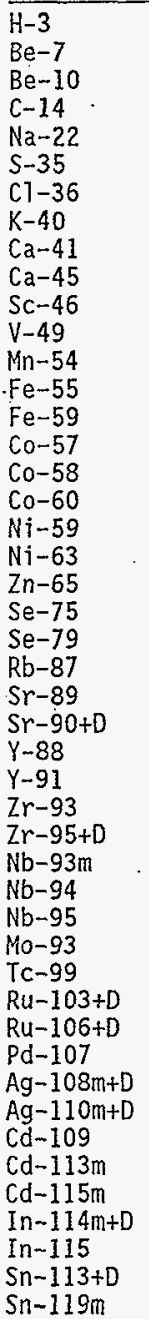 & $\begin{array}{l}2.68 \mathrm{E}-06 \\
1.47 \mathrm{E}-06 \\
4.37 \mathrm{E}-03 \\
7.72 \mathrm{E}-05 \\
3.32 \mathrm{E}-04 \\
1.57 \mathrm{E}-05 \\
3.39 \mathrm{E}-04 \\
6.13 \mathrm{E}-04 \\
4.80 \mathrm{E}-05 \\
8.05 \mathrm{E}-05 \\
1.62 \mathrm{E}-04 \\
3.96 \mathrm{E}-06 \\
1.03 \mathrm{E}-04 \\
4.18 \mathrm{E}-05 \\
6.06 \mathrm{E}-05 \\
8.99 \mathrm{E}-05 \\
5.98 \mathrm{E}-05 \\
3.09 \mathrm{E}-03 \\
2.11 \mathrm{E}-05 \\
5.15 \mathrm{E}-05 \\
3.77 \mathrm{E}-04 \\
1.43 \mathrm{E}-04 \\
3.35 \mathrm{E}-04 \\
1.62 \mathrm{E}-04 \\
6.14 \mathrm{E}-05 \\
6.72 \mathrm{E}-03 \\
1.86 \mathrm{E}-04 \\
1.88 \mathrm{E}-04 \\
3.90 \mathrm{E}-03 \\
1.26 \mathrm{E}-04 \\
3.55 \mathrm{E}-04 \\
5.15 \mathrm{E}-03 \\
1.86 \mathrm{E}-05 \\
3.83 \mathrm{E}-04 \\
1.36 \mathrm{E}-04 \\
2.86 \mathrm{E}-05 \\
4.65 \mathrm{E}-03 \\
1.57 \mathrm{E}-04 \\
3.58 \mathrm{E}-03 \\
7.76 \mathrm{E}-04 \\
1.31 \mathrm{E}-03 \\
2.18 \mathrm{E}-02 \\
2.23 \mathrm{E}-04 \\
2.90 \mathrm{E}-04 \\
4.88 \mathrm{E}-02 \\
8.41 \mathrm{E}-05 \\
7.35 \mathrm{E}-05\end{array}$ & $\begin{array}{l}\text { Ingest } \\
\text { Inhale } \\
\text { Inhale } \\
\text { Ingest } \\
\text { Ingest } \\
\text { Inhale } \\
\text { Inhale } \\
\text { Ingest } \\
\text { Ingest } \\
\text { Inhale } \\
\text { Inhale } \\
\text { Inhale } \\
\text { Inhale } \\
\text { Inhale } \\
\text { Inhale } \\
\text { Inhale } \\
\text { Inhale } \\
\text { Inhale } \\
\text { Inhale } \\
\text { Inhale } \\
\text { Ingest } \\
\text { Ingest } \\
\text { Ingest } \\
\text { Ingest } \\
\text { Ingest } \\
\text { Ingest } \\
\text { Inhale } \\
\text { Inhale } \\
\text { Inhale } \\
\text { Inhale } \\
\text { Inhale } \\
\text { Inhale } \\
\text { Inhale } \\
\text { Inhale } \\
\text { Inhale } \\
\text { Inhale } \\
\text { Inhale } \\
\text { Inhale } \\
\text { Inhale } \\
\text { Inhale } \\
\text { Inhale } \\
\text { Inhale } \\
\text { Inhale } \\
\text { Inhale } \\
\text { Inhale } \\
\text { Inhale } \\
\text { Inhale }\end{array}$ & $\begin{array}{l}3.74 E+05 \\
6.79 E+05 \\
2.29 E+02 \\
1.30 E+04 \\
3.01 E+03 \\
6.39 E+04 \\
2.95 E+03 \\
1.63 E+03 \\
2.08 E+04 \\
1.24 E+04 \\
6.16 E+03 \\
2.52 E+05 \\
9.75 E+03 \\
2.39 E+04 \\
1.65 E+04 \\
1.11 E+04 \\
1.67 E+04 \\
3.24 E+02 \\
4.73 E+04 \\
1.94 E+04 \\
2.65 E+03 \\
7.02 E+03 \\
2.98 E+03 \\
6.18 E+03 \\
1.63 E+04 \\
1.49 E+02 \\
5.38 E+03 \\
5.32 E+03 \\
2.56 E+02 \\
7.91 E+03 \\
2.81 E+03 \\
1.94 E+02 \\
5.39 E+04 \\
2.61 E+03 \\
7.33 E+03 \\
3.50 E+04 \\
2.15 E+02 \\
6.37 E+03 \\
2.79 E+02 \\
1.29 E+03 \\
7.63 E+02 \\
4.59 E+01 \\
4.48 E+03 \\
3.45 E+03 \\
2.05 E+01 \\
1.19 E+04 \\
1.36 E+04\end{array}$ & $\begin{array}{l}3.74 E+07 \\
6.79 E+07 \\
2.29 E+04 \\
1.30 E+06 \\
3.01 E+05 \\
6.39 E+06 \\
2.95 E+05 \\
1.63 E+05 \\
2.08 E+06 \\
1.24 E+06 \\
6.16 E+05 \\
2.52 E+07 \\
9.75 E+05 \\
2.39 E+06 \\
1.65 E+06 \\
1.11 E+06 \\
1.67 E+06 \\
3.24 E+04 \\
4.73 E+06 \\
1.94 E+06 \\
2.65 E+05 \\
7.02 E+05 \\
2.98 E+05 \\
6.18 E+05 \\
1.63 E+06 \\
1.49 E+04 \\
5.38 E+05 \\
5.32 E+05 \\
2.56 E+04 \\
7.91 E+05 \\
2.81 E+05 \\
1.94 E+04 \\
5.39 E+06 \\
2.61 E+05 \\
7.33 E+05 \\
3.50 E+06 \\
2.15 E+04 \\
6.37 E+05 \\
2.79 E+04 \\
1.29 E+05 \\
7.63 E+04 \\
4.59 E+03 \\
4.48 E+05 \\
3.45 E+05 \\
2.05 E+03 \\
1.19 E+06 \\
1.36 E+05\end{array}$ & $\begin{array}{l}1.87 E+08 \\
3.40 E+08 \\
1.14 E+05 \\
6.48 E+06 \\
1.50 E+06 \\
3.19 E+07 \\
1.48 E+06 \\
8.16 E+05 \\
1.04 E+07 \\
6.21 E+06 \\
3.08 E+06 \\
1.26 E+08 \\
4.88 E+06 \\
1.20 E+07 \\
8.25 E+06 \\
5.56 E+06 \\
8.36 E+06 \\
1.62 E+05 \\
2.36 E+07 \\
9.71 E+06 \\
1.33 E+06 \\
3.51 E+06 \\
1.49 E+06 \\
3.09 E+06 \\
8.14 E+06 \\
7.44 E+04 \\
2.69 E+06 \\
2.66 E+06 \\
1.28 E+05 \\
3.96 E+06 \\
1.41 E+06 \\
9.71 E+04 \\
2.69 E+07 \\
1.30 E+06 \\
3.66 E+06 \\
1.75 E+07 \\
1.08 E+05 \\
3.19 E+06 \\
1.40 E+05 \\
6.45 E+05 \\
3.82 E+05 \\
2.29 E+04 \\
2.24 E+06 \\
1.72 E+06 \\
1.02 E+04 \\
5.95 E+06 \\
6.80 E+06\end{array}$ \\
\hline
\end{tabular}


Table 1. Unit Dose Factors and Soil Concentration Limits, Continued

\begin{tabular}{|c|c|c|c|c|c|}
\hline Nucl ide & $\begin{array}{l}\text { EDE mrem } / y \\
\text { at } 1 \mathrm{pCi} / \mathrm{g}\end{array}$ & $\begin{array}{l}\text { Max } \\
\text { Pathway }\end{array}$ & $\begin{array}{l}\mathrm{pCi} / \mathrm{g} \text { to } \\
1 \text { mrem }\end{array}$ & $\begin{array}{l}\text { ive the an } \\
100 \text { mrem }\end{array}$ & $\begin{array}{l}\text { ual doses } \\
500 \text { mrem }\end{array}$ \\
\hline 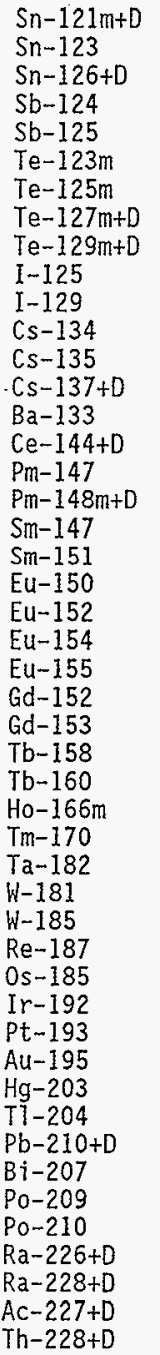 & $\begin{array}{l}1.98 \mathrm{E}-04 \\
2.63 \mathrm{E}-04 \\
1.74 \mathrm{E}-03 \\
1.30 \mathrm{E}-04 \\
2.21 \mathrm{E}-04 \\
1.12 \mathrm{E}-04 \\
4.05 \mathrm{E}-05 \\
1.88 \mathrm{E}-04 \\
7.41 \mathrm{E}-05 \\
2.93 \mathrm{E}-04 \\
8.98 \mathrm{E}-03 \\
2.03 \mathrm{E}-03 \\
2.31 \mathrm{E}-04 \\
1.61 \mathrm{E}-03 \\
1.73 \mathrm{E}-04 \\
3.32 \mathrm{E}-03 \\
4.37 \mathrm{E}-04 \\
7.84 \mathrm{E}-05 \\
9.02 \mathrm{E}-01 \\
3.68 \mathrm{E}-04 \\
3.35 \mathrm{E}-03 \\
2.74 \mathrm{E}-03 \\
3.53 \mathrm{E}-03 \\
5.00 \mathrm{E}-04 \\
2.93 \mathrm{E}+00 \\
1.95 \mathrm{E}-04 \\
3.17 \mathrm{E}-03 \\
1.30 \mathrm{E}-04 \\
9.48 \mathrm{E}-03 \\
1.96 \mathrm{E}-04 \\
2.83 \mathrm{E}-04 \\
4.37 \mathrm{E}-06 \\
1.68 \mathrm{E}-05 \\
8.90 \mathrm{E}-07 \\
6.24 \mathrm{E}-05 \\
1.36 \mathrm{E}-04 \\
5.66 \mathrm{E}-06 \\
9.85 \mathrm{E}-05 \\
6.85 \mathrm{E}-05 \\
1.03 \mathrm{E}-04 \\
3.81 \mathrm{E}-01 \\
3.73 \mathrm{E}-04 \\
2.00 \mathrm{E}-01 \\
7.36 \mathrm{E}-02 \\
1.42 \mathrm{E}-01 \\
7.97 \mathrm{E}-001 \\
3.49 \mathrm{E}+00\end{array}$ & $\begin{array}{l}\text { Inhale } \\
\text { Inhale } \\
\text { Inhale } \\
\text { Inhale } \\
\text { Inhale } \\
\text { Ingest } \\
\text { Ingest } \\
\text { Inhale } \\
\text { Inhale } \\
\text { Ingest } \\
\text { Ingest } \\
\text { Ingest } \\
\text { Ingest } \\
\text { Ingest } \\
\text { Inhale } \\
\text { Inhale } \\
\text { Inhale } \\
\text { Inhale } \\
\text { Inhale } \\
\text { Inhale } \\
\text { Inhale } \\
\text { Inhale } \\
\text { Inhale } \\
\text { Inhale } \\
\text { Inhale } \\
\text { Inhale } \\
\text { Inhale } \\
\text { Inhale } \\
\text { Inhale } \\
\text { Inhale } \\
\text { Inhale } \\
\text { Ingest } \\
\text { Ingest } \\
\text { Inhale } \\
\text { Inhale } \\
\text { Inhale } \\
\text { Ingest } \\
\text { Inhale } \\
\text { Ingest } \\
\text { Ingest } \\
\text { Inhale } \\
\text { Inhale } \\
\text { Inhale } \\
\text { Inhale } \\
\text { Inhale } \\
\text { Inhale } \\
\text { Inhale } \\
\text { Inhale }\end{array}$ & $\begin{array}{l}5.05 E+03 \\
3.80 E+03 \\
5.74 E+02 \\
7.69 E+03 \\
4.53 E+03 \\
8.96 E+03 \\
2.47 E+04 \\
5.31 E+03 \\
1.35 E+04 \\
3.42 E+03 \\
1.11 E+02 \\
4.93 E+02 \\
4.32 E+03 \\
6.20 E+02 \\
5.78 E+03 \\
3.01 E+02 \\
2.29 E+03 \\
1.28 E+04 \\
1.11 E+00 \\
2.72 E+03 \\
2.99 E+02 \\
3.65 E+02 \\
2.83 E+02 \\
2.00 E+03 \\
3.42 E-01 \\
5.12 E+03 \\
3.15 E+02 \\
7.71 E+03 \\
1.06 E+02 \\
5.10 E+03 \\
3.54 E+03 \\
2.29 E+05 \\
5.94 E+04 \\
1.12 E+06 \\
1.60 E+04 \\
7.35 E+03 \\
1.77 E+05 \\
1.01 E+04 \\
1.46 E+04 \\
9.70 E+03 \\
2.63 E+00 \\
2.68 E+03 \\
5.00 E+00 \\
1.36 E+01 \\
7.04 E+00 \\
1.36 E+00 \\
1.25 E-02 \\
2.87 E-01\end{array}$ & $\begin{array}{l}5.05 E+05 \\
3.80 E+05 \\
5.74 E+04 \\
7.69 E+05 \\
4.53 E+05 \\
8.96 E+05 \\
2.47 E+06 \\
5.31 E+05 \\
1.35 E+06 \\
3.42 E+05 \\
1.11 E+04 \\
4.93 E+04 \\
4.32 E+05 \\
6.20 E+04 \\
5.78 E+05 \\
3.01 E+04 \\
2.29 E+05 \\
1.28 E+06 \\
1.11 E+02 \\
2.72 E+05 \\
2.99 E+04 \\
3.65 E+04 \\
2.83 E+04 \\
2.00 E+05 \\
3.42 E+01 \\
5.12 E+05 \\
3.15 E+04 \\
7.71 E+05 \\
1.06 E+04 \\
5.10 E+05 \\
3.54 E+05 \\
2.29 E+07 \\
5.94 E+06 \\
1.12 E+08 \\
1.60 E+06 \\
7.35 E+05 \\
1.77 E+07 \\
1.01 E+06 \\
1.46 E+06 \\
9.70 E+05 \\
2.63 E+02 \\
2.68 E+05 \\
5.00 E+02 \\
1.36 E+03 \\
7.04 E+02 \\
1.36 E+02 \\
1.25 E+00 \\
2.87 E+01\end{array}$ & $\begin{array}{l}2.53 \mathrm{E}+06 \\
1.90 \mathrm{E}+06 \\
2.87 \mathrm{E}+05 \\
3.84 \mathrm{E}+06 \\
2.27 \mathrm{E}+06 \\
4.48 \mathrm{E}+06 \\
1.23 \mathrm{E}+07 \\
2.65 \mathrm{E}+06 \\
6.75 \mathrm{E}+06 \\
1.71 \mathrm{E}+06 \\
5.57 \mathrm{E}+04 \\
2.47 \mathrm{E}+05 \\
2.16 \mathrm{E}+06 \\
3.10 \mathrm{E}+05 \\
2.89 \mathrm{E}+06 \\
1.51 \mathrm{E}+05 \\
1.15 \mathrm{E}+06 \\
6.38 \mathrm{E}+06 \\
5.55 \mathrm{E}+02 \\
1.36 \mathrm{E}+06 \\
1.49 \mathrm{E}+05 \\
1.82 \mathrm{E}+05 \\
1.42 \mathrm{E}+05 \\
1.00 \mathrm{E}+06 \\
1.71 \mathrm{E}+02 \\
2.56 \mathrm{E}+06 \\
1.58 \mathrm{E}+05 \\
3.86 \mathrm{E}+06 \\
5.28 \mathrm{E}+04 \\
2.55 \mathrm{E}+06 \\
1.77 \mathrm{E}+06 \\
1.14 \mathrm{E}+08 \\
2.97 \mathrm{E}+07 \\
5.62 \mathrm{E}+08 \\
8.01 \mathrm{E}+06 \\
3.67 \mathrm{E}+06 \\
8.84 \mathrm{E}+07 \\
5.07 \mathrm{E}+06 \\
7.30 \mathrm{E}+06 \\
4.85 \mathrm{E}+06 \\
1.31 \mathrm{E}+03 \\
1.34 \mathrm{E}+06 \\
2.50 \mathrm{E}+03 \\
6.80 \mathrm{E}+03 \\
3.52 \mathrm{E}+03 \\
6.81 \mathrm{E}+02 \\
6.27 \mathrm{E}+00 \\
1.43 \mathrm{E}+02\end{array}$ \\
\hline
\end{tabular}


Tabie 1. Unit Dose Factors and Soil Concentration Limits, Continued

\begin{tabular}{|c|c|c|c|c|c|}
\hline Nuclide & $\begin{array}{l}\text { TEDE mrem/y } \\
\text { at } 1 \mathrm{pCi} / \mathrm{g}\end{array}$ & $\begin{array}{c}\text { Max } \\
\text { Pathway }\end{array}$ & $\begin{array}{c}\mathrm{pCi} / \mathrm{g} \text { to } \\
1 \text { mrem }\end{array}$ & $\begin{array}{l}\text { ive the an } \\
100 \text { mrem }\end{array}$ & $\begin{array}{l}\text { ial doses } \\
500 \text { mrem }\end{array}$ \\
\hline $\begin{array}{l}\text { Th-229+D } \\
\text { Th-230 } \\
\text { Th-232 } \\
P a-231 \\
U-232 \\
U-233 \\
U-234 \\
U-235+D \\
U-236 \\
U-238+D \\
N p-236 \\
N p-237+D \\
P u-236 \\
P u-237 \\
P u-238 \\
P u-239 \\
P u-240 \\
P u-241+D \\
P u-242 \\
P u-244+D \\
\text { Am-241 } \\
\text { Am-242m+D } \\
\text { Am-243+D } \\
\text { Cm-242 } \\
\text { Cm-243 } \\
\text { Cm-244 } \\
\text { Cm-245 } \\
\text { Cm-246 } \\
\text { Cm-247+D } \\
\text { Cm-248 } \\
\text { Cf }-250 \\
C f-252 \\
\text { Es }-254+D\end{array}$ & $\begin{array}{l}2.61 \mathrm{E}+01 \\
3.92 \mathrm{E}+00 \\
1.98 \mathrm{E}+01 \\
1.69 \mathrm{E}+01 \\
8.78 \mathrm{E}-01 \\
1.04 \mathrm{E}-01 \\
1.02 \mathrm{E}-01 \\
9.43 \mathrm{E}-02 \\
9.60 \mathrm{E}-02 \\
9.14 \mathrm{E}-02 \\
1.29 \mathrm{E}+00 \\
6.59 \mathrm{E}+00 \\
1.57 \mathrm{E}+00 \\
6.50 \mathrm{E}-06 \\
4.77 \mathrm{E}+00 \\
5.24 \mathrm{E}+00 \\
5.24 \mathrm{E}+00 \\
1.03 \mathrm{E}-01 \\
5.01 \mathrm{E}+00 \\
4.92 \mathrm{E}+00 \\
5.41 \mathrm{E}+00 \\
5.27 \mathrm{E}+00 \\
5.37 \mathrm{E}+00 \\
1.19 \mathrm{E}-01 \\
3.70 \mathrm{E}+00 \\
2.97 \mathrm{E}+00 \\
5.56 \mathrm{E}+00 \\
5.51 \mathrm{E}+00 \\
5.06 \mathrm{E}+00 \\
2.02 \mathrm{E}+01 \\
3.11 \mathrm{E}+00 \\
1.68 \mathrm{E}+00 \\
3.90 \mathrm{E}-01\end{array}$ & $\begin{array}{l}\text { Inhale } \\
\text { Inhale } \\
\text { Inhale } \\
\text { Inhale } \\
\text { Inhale } \\
\text { Inhale } \\
\text { Inhale } \\
\text { Inhale } \\
\text { Inhale } \\
\text { Inhale } \\
\text { Inhale } \\
\text { Inhale } \\
\text { Inhale } \\
\text { Inhale } \\
\text { Inhale } \\
\text { Inhale } \\
\text { Inhale } \\
\text { Inhale } \\
\text { Inhale } \\
\text { Inhale } \\
\text { Inhale } \\
\text { Inhale } \\
\text { Inhale } \\
\text { Inhale } \\
\text { Inhale } \\
\text { Inhale } \\
\text { Inhale } \\
\text { Inhale } \\
\text { Inhale } \\
\text { Inhale } \\
\text { Inhale } \\
\text { Inhale } \\
\text { Inhale }\end{array}$ & $\begin{array}{l}3.83 \mathrm{E}-02 \\
2.55 \mathrm{E}-01 \\
5.06 \mathrm{E}-02 \\
5.90 \mathrm{E}-02 \\
1.14 \mathrm{E}+00 \\
9.58 \mathrm{E}+00 \\
9.84 \mathrm{E}+00 \\
1.06 \mathrm{E}+01 \\
1.04 \mathrm{E}+01 \\
1.09 \mathrm{E}+01 \\
7.77 \mathrm{E}-01 \\
1.52 \mathrm{E}-01 \\
6.37 \mathrm{E}-01 \\
1.54 \mathrm{E}+05 \\
2.10 \mathrm{E}-01 \\
1.91 \mathrm{E}-01 \\
1.91 \mathrm{E}-01 \\
9.75 \mathrm{E}+00 \\
2.00 \mathrm{E}-01 \\
2.03 \mathrm{E}-01 \\
1.85 \mathrm{E}-01 \\
1.90 \mathrm{E}-01 \\
1.86 \mathrm{E}-01 \\
8.43 \mathrm{E}+00 \\
2.70 \mathrm{E}-01 \\
3.37 \mathrm{E}-01 \\
1.80 \mathrm{E}-01 \\
1.82 \mathrm{E}-01 \\
1.98 \mathrm{E}-01 \\
4.95 \mathrm{E}-02 \\
3.21 \mathrm{E}-01 \\
5.95 \mathrm{E}-01 \\
2.56 \mathrm{E}+00\end{array}$ & $\begin{array}{l}3.83 E+00 \\
2.55 E+01 \\
5.06 E+00 \\
5.90 E+00 \\
1.14 E+02 \\
9.58 E+02 \\
9.84 E+02 \\
1.06 E+03 \\
1.04 E+03 \\
1.09 E+03 \\
7.77 E+01 \\
1.52 E+01 \\
6.37 E+01 \\
1.54 E+07 \\
2.10 E+01 \\
1.91 E+01 \\
1.91 E+01 \\
9.75 E+02 \\
2.00 E+01 \\
2.03 E+01 \\
1.85 E+01 \\
1.90 E+01 \\
1.86 E+01 \\
8.43 E+02 \\
2.70 E+01 \\
3.37 E+01 \\
1.80 E+01 \\
1.82 E+01 \\
1.98 E+01 \\
4.95 E+00 \\
3.21 E+01 \\
5.95 E+01 \\
2.56 E+02\end{array}$ & $\begin{array}{l}1.92 \mathrm{E}+01 \\
1.28 \mathrm{E}+02 \\
2.53 \mathrm{E}+01 \\
2.95 \mathrm{E}+01 \\
5.69 \mathrm{E}+02 \\
4.79 \mathrm{E}+03 \\
4.92 \mathrm{E}+03 \\
5.30 \mathrm{E}+03 \\
5.21 \mathrm{E}+03 \\
5.47 \mathrm{E}+03 \\
3.88 \mathrm{E}+02 \\
7.58 \mathrm{E}+01 \\
3.18 \mathrm{E}+02 \\
7.70 \mathrm{E}+07 \\
1.05 \mathrm{E}+02 \\
9.54 \mathrm{E}+01 \\
9.54 \mathrm{E}+01 \\
4.87 \mathrm{E}+03 \\
9.98 \mathrm{E}+01 \\
1.02 \mathrm{E}+02 \\
9.23 \mathrm{E}+01 \\
9.48 \mathrm{E}+01 \\
9.30 \mathrm{E}+01 \\
4.21 \mathrm{E}+03 \\
1.35 \mathrm{E}+02 \\
1.68 \mathrm{E}+02 \\
9.00 \mathrm{E}+01 \\
9.08 \mathrm{E}+01 \\
9.88 \mathrm{E}+01 \\
2.48 \mathrm{E}+01 \\
1.61 \mathrm{E}+02 \\
2.98 \mathrm{E}+02 \\
1.28 \mathrm{E}+03\end{array}$ \\
\hline 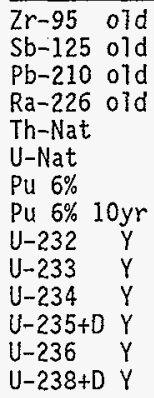 & $\begin{array}{l}1.67 E-04 \\
2.31 E-04 \\
4.55 E-01 \\
6.03 E-01 \\
2.40 E+01 \\
4.71 E+00 \\
5.95 E+00 \\
5.65 E+00 \\
8.57 E+00 \\
1.63 E+00 \\
1.60 E+00 \\
1.48 E+00 \\
1.51 E+00 \\
1.43 E+00\end{array}$ & $\begin{array}{l}\text { Inhale } \\
\text { Inhale } \\
\text { Inhale } \\
\text { Inhale } \\
\text { Inhale } \\
\text { Inhale } \\
\text { Inhale } \\
\text { Inhale } \\
\text { Inhale } \\
\text { Inhale } \\
\text { Inhale } \\
\text { Inhale } \\
\text { Inhale } \\
\text { Inhale }\end{array}$ & $\begin{array}{l}5.97 \mathrm{E}+03 \\
4.34 \mathrm{E}+03 \\
2.20 \mathrm{E}+00 \\
1.66 \mathrm{E}+00 \\
4.17 \mathrm{E}-02 \\
2.12 \mathrm{E}-01 \\
1.68 \mathrm{E}-01 \\
1.77 \mathrm{E}-01 \\
1.17 \mathrm{E}-01 \\
6.12 \mathrm{E}-01 \\
6.26 \mathrm{E}-01 \\
6.75 \mathrm{E}-01 \\
6.62 \mathrm{E}-01 \\
7.01 \mathrm{E}-01\end{array}$ & $\begin{array}{l}5.97 E+05 \\
4.34 E+05 \\
2.20 E+02 \\
1.66 E+02 \\
4.17 E+00 \\
2.12 E+01 \\
1.68 E+01 \\
1.77 E+01 \\
1.17 E+01 \\
6.12 E+01 \\
6.26 E+01 \\
6.75 E+01 \\
6.62 E+01 \\
7.01 E+01\end{array}$ & $\begin{array}{l}2.99 E+06 \\
2.17 E+06 \\
1.10 E+03 \\
8.30 E+02 \\
2.08 E+01 \\
1.06 E+02 \\
8.41 E+01 \\
8.84 E+01 \\
5.84 E+01 \\
3.06 E+02 \\
3.13 E+02 \\
3.38 E+02 \\
3.31 E+02 \\
3.50 E+02\end{array}$ \\
\hline
\end{tabular}


Table 2. Soil Limits and Instrument Response - $1 \mathrm{mrem} / \mathrm{y}$

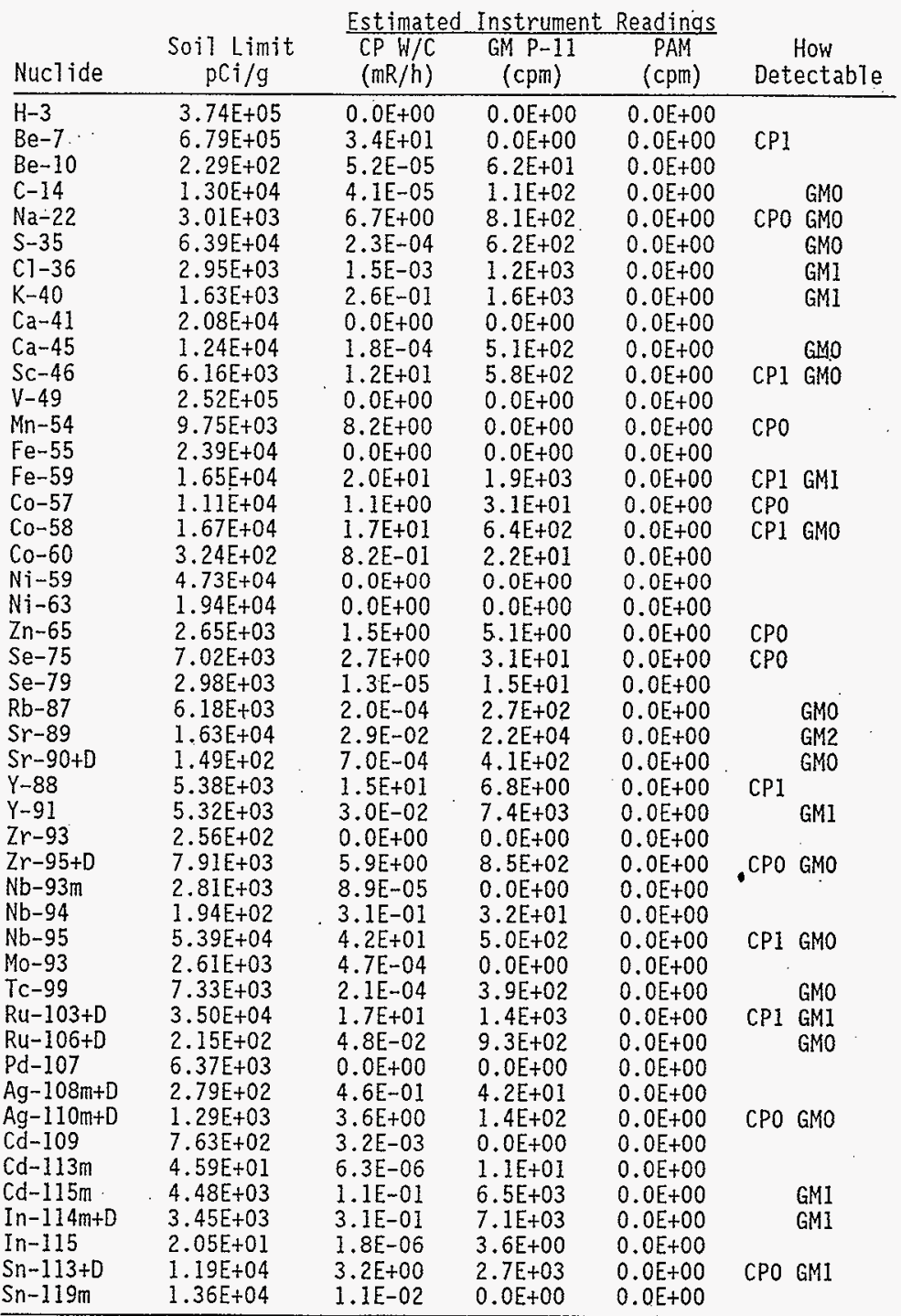


Table 2. Soil Limits and Instrument Response $-1 \mathrm{mrem} / \mathrm{y}$, Continued

\begin{tabular}{|c|c|c|c|c|c|c|}
\hline Nucl ide & $\begin{array}{l}\text { Soil Limit } \\
\mathrm{pCi} / \mathrm{g}\end{array}$ & $\begin{array}{l}\frac{\text { Estimate }}{\mathrm{CP} W / \mathrm{C}} \\
(\mathrm{mR} / \mathrm{h})\end{array}$ & $\begin{array}{c}\text { instrumen } \\
\text { GM P-11 } \\
\text { (cpm) }\end{array}$ & 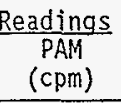 & Dete & $\begin{array}{l}\text { How } \\
\text { ectabre }\end{array}$ \\
\hline 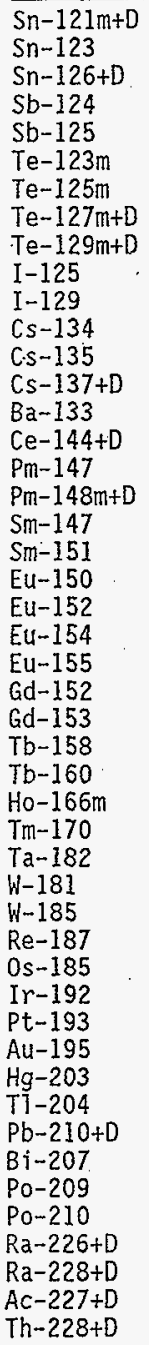 & $\begin{array}{l}5.05 E+03 \\
3.80 E+03 \\
5.74 E+02 \\
7.69 E+03 \\
4.53 E+03 \\
8.96 E+03 \\
2.47 E+04 \\
5.31 E+03 \\
1.35 E+04 \\
3.42 E+03 \\
1.11 E+02 \\
4.93 E+02 \\
4.32 E+03 \\
6.20 E+02 \\
5.78 E+03 \\
3.01 E+02 \\
2.29 E+03 \\
1.28 E+04 \\
1.11 E+00 \\
2.72 E+03 \\
2.99 E+02 \\
3.65 E+02 \\
2.83 E+02 \\
2.00 E+03 \\
3.42 E-01 \\
5.12 E+03 \\
3.15 E+02 \\
7.71 E+03 \\
1.06 E+02 \\
5.10 E+03 \\
3.54 E+03 \\
2.29 E+05 \\
5.94 E+04 \\
1.12 E+06 \\
1.60 E+04 \\
7.35 E+03 \\
1.77 E+05 \\
1.01 E+04 \\
1.46 E+04 \\
9.70 E+03 \\
2.63 E+00 \\
2.68 E+03 \\
5.00 E+00 \\
1.36 E+01 \\
7.04 E+00 \\
1.36 E+00 \\
1.25 E-02 \\
2.87 E-01\end{array}$ & $\begin{array}{l}2.8 \mathrm{E}-03 \\
3.2 \mathrm{E}-02 \\
1.2 \mathrm{E}+00 \\
1.4 \mathrm{E}+01 \\
1.9 \mathrm{E}+00 \\
1.1 \mathrm{E}+00 \\
9.9 \mathrm{E}-02 \\
3.4 \mathrm{E}-02 \\
9.2 \mathrm{E}-01 \\
1.6 \mathrm{E}-02 \\
3.9 \mathrm{E}-04 \\
7.9 \mathrm{E}-01 \\
3.8 \mathrm{E}-05 \\
3.6 \mathrm{E}-01 \\
2.2 \mathrm{E}+00 \\
1.7 \mathrm{E}-02 \\
2.6 \mathrm{E}-05 \\
2.6 \mathrm{E}+01 \\
0.0 \mathrm{E}+00 \\
7.5 \mathrm{E}-07 \\
4.5 \mathrm{E}-01 \\
4.1 \mathrm{E}-01 \\
3.5 \mathrm{E}-01 \\
7.9 \mathrm{E}-02 \\
0.0 \mathrm{E}+00 \\
2.7 \mathrm{E}-01 \\
2.4 \mathrm{E}-01 \\
8.7 \mathrm{E}+00 \\
1.9 \mathrm{E}-01 \\
1.7 \mathrm{E}-02 \\
4.5 \mathrm{E}+00 \\
4.1 \mathrm{E}+00 \\
5.5 \mathrm{E}-03 \\
0.0 \mathrm{E}+00 \\
1.1 \mathrm{E}+01 \\
6.2 \mathrm{E}+00 \\
0.0 \mathrm{E}+00 \\
4.7 \mathrm{E}-01 \\
3.5 \mathrm{E}+00 \\
8.6 \mathrm{E}-03 \\
3.4 \mathrm{E}-06 \\
4.1 \mathrm{E}+00 \\
1.5 \mathrm{E}-01 \\
1.2 \mathrm{E}-07 \\
1.3 \mathrm{E}-02 \\
1.3 \mathrm{E}-03 \\
4.8 \mathrm{E}-06 \\
4.6 \mathrm{E}-04\end{array}$ & $\begin{array}{l}1.3 E+02 \\
4.4 E+03 \\
9.1 E+02 \\
6.5 E+03 \\
3.4 E+02 \\
2.2 E+02 \\
6.7 E+02 \\
1.8 E+03 \\
1.7 E+04 \\
0.0 E+00 \\
5.8 E-01 \\
1.1 E+02 \\
7.6 E+01 \\
2.2 E+02 \\
7.5 E+01 \\
1.1 E+03 \\
5.4 E+01 \\
3.7 E+03 \\
0.0 E+00 \\
4.0 E-04 \\
0.0 E+00 \\
6.4 E+01 \\
1.2 E+02 \\
2.1 E+01 \\
0.0 E+00 \\
1.3 E+01 \\
2.9 E+01 \\
2.8 E+03 \\
1.0 E+01 \\
2.9 E+03 \\
5.2 E+02 \\
0.0 E+00 \\
7.4 E+03 \\
0.0 E+00 \\
2.0 E+02 \\
2.1 E+03 \\
0.0 E+00 \\
3.8 E+01 \\
1.1 E+03 \\
3.6 E+03 \\
2.1 E+00 \\
7.3 E+02 \\
1.1 E-03 \\
0.0 E+00 \\
1.5 E+01 \\
1.1 E+00 \\
2.8 E-02 \\
5.4 E-01\end{array}$ & $\begin{array}{l}0.0 E+00 \\
0.0 E+00 \\
0.0 E+00 \\
0.0 E+00 \\
0.0 E+00 \\
0.0 E+00 \\
0.0 E+00 \\
0.0 E+00 \\
0.0 E+00 \\
0.0 E+00 \\
0.0 E+00 \\
0.0 E+00 \\
0.0 E+00 \\
0.0 E+00 \\
0.0 E+00 \\
0.0 E+00 \\
0.0 E+00 \\
0.0 E+00 \\
0.0 E+00 \\
0.0 E+00 \\
0.0 E+00 \\
0.0 E+00 \\
0.0 E+00 \\
0.0 E+00 \\
0.0 E+00 \\
0.0 E+00 \\
0.0 E+00 \\
0.0 E+00 \\
0.0 E+00 \\
0.0 E+00 \\
0.0 E+00 \\
0.0 E+00 \\
0.0 E+00 \\
0.0 E+00 \\
0.0 E+00 \\
0.0 E+00 \\
0.0 E+00 \\
0.0 E+00 \\
0.0 E+00 \\
0.0 E+00 \\
0.0 E+00 \\
0.0 E+00 \\
2.6 E-01 \\
8.8 E-01 \\
2.5 E+00 \\
0.0 E+00 \\
6.7 E-03 \\
1.5 E-01\end{array}$ & $\begin{array}{l}\text { CPO } \\
\text { CPO } \\
\text { CP1 } \\
\text { CPO }\end{array}$ & $\begin{array}{l}\text { GMO } \\
\text { GM1 } \\
\text { GMO } \\
\text { GM1 } \\
\text { GMO } \\
\text { GMO } \\
\text { GMO } \\
\text { GMI } \\
\text { GM2 } \\
\text { GMO } \\
\text { GMO } \\
\text { GM1 } \\
\text { GMI }\end{array}$ \\
\hline
\end{tabular}


Table 2. Soi1 Limits and Instrument Response - $1 \mathrm{mrem} / \mathrm{y}$, Continued

\begin{tabular}{|c|c|c|c|c|c|c|}
\hline \multirow[b]{2}{*}{ Nuclide } & \multicolumn{6}{|c|}{ Estimated Instrument Readings } \\
\hline & $\begin{array}{c}\text { Soil Limit } \\
\mathrm{pCi} / \mathrm{g}\end{array}$ & $\begin{array}{l}C P W / C \\
(m R / h)\end{array}$ & $\begin{array}{c}\text { GM P } P-11 \\
(\mathrm{cpm})\end{array}$ & $\begin{array}{c}\text { PAM } \\
\text { (cpm) }\end{array}$ & $\begin{array}{r}H \\
\text { Dete }\end{array}$ & $\begin{array}{l}\text { How } \\
\text { ectable }\end{array}$ \\
\hline $\begin{array}{l}\text { Th-229+D } \\
\text { Th-230 } \\
T h-232 \\
\mathrm{~Pa}-231 \\
U-232 \\
U-233 \\
U-234 \\
U-235+D \\
U-236 \\
U-238+D \\
\mathrm{~Np}-236 \\
\mathrm{~Np}-237+D \\
\mathrm{Pu}-236 \\
\mathrm{Pu}-237 \\
\mathrm{Pu}-238 \\
\mathrm{Pu}-239 \\
\mathrm{Pu}-240 \\
\mathrm{Pu}-241+\mathrm{D} \\
\mathrm{Pu}-242 \\
\mathrm{Pu}-244+\mathrm{D} \\
\mathrm{Am}-241 \\
\mathrm{Am}-242 \mathrm{~m}+\mathrm{D} \\
\mathrm{Am}-243+\mathrm{D} \\
\mathrm{Cm}-242 \\
\mathrm{Cm}-243 \\
\mathrm{Cm}-244 \\
\mathrm{Cm}-245 \\
\mathrm{Cm}-246 \\
\mathrm{Cm}-247+\mathrm{D} \\
\mathrm{Cm}-248 \\
\mathrm{Cf}-250 \\
\mathrm{Cf}-252 \\
\mathrm{Es}-254+\mathrm{D}\end{array}$ & $\begin{array}{l}3.83 \mathrm{E}-02 \\
2.55 \mathrm{E}-01 \\
5.06 \mathrm{E}-02 \\
5.90 \mathrm{E}-02 \\
1.14 \mathrm{E}+00 \\
9.58 \mathrm{E}+00 \\
9.84 \mathrm{E}+00 \\
1.06 \mathrm{E}+01 \\
1.04 \mathrm{E}+01 \\
1.09 \mathrm{E}+01 \\
7.77 \mathrm{E}-01 \\
1.52 \mathrm{E}-01 \\
6.37 \mathrm{E}-01 \\
1.54 \mathrm{E}+05 \\
2.10 \mathrm{E}-01 \\
1.91 \mathrm{E}-01 \\
1.91 \mathrm{E}-01 \\
9.75 \mathrm{E}+00 \\
2.00 \mathrm{E}-01 \\
2.03 \mathrm{E}-01 \\
1.85 \mathrm{E}-01 \\
1.90 \mathrm{E}-01 \\
1.86 \mathrm{E}-01 \\
8.43 \mathrm{E}+00 \\
2.70 \mathrm{E}-01 \\
3.37 \mathrm{E}-01 \\
1.80 \mathrm{E}-01 \\
1.82 \mathrm{E}-01 \\
1.98 \mathrm{E}-01 \\
4.95 \mathrm{E}-02 \\
3.21 \mathrm{E}-01 \\
5.95 \mathrm{E}-01 \\
2.56 \mathrm{E}+00\end{array}$ & $\begin{array}{l}1.1 \mathrm{E}-05 \\
6.6 \mathrm{E}-08 \\
5.8 \mathrm{E}-09 \\
2.1 \mathrm{E}-06 \\
2.2 \mathrm{E}-07 \\
2.7 \mathrm{E}-06 \\
8.7 \mathrm{E}-07 \\
1.6 \mathrm{E}-03 \\
5.2 \mathrm{E}-07 \\
2.6 \mathrm{E}-04 \\
7.3 \mathrm{E}-05 \\
3.2 \mathrm{E}-05 \\
3.5 \mathrm{E}-08 \\
5.2 \mathrm{E}+00 \\
8.2 \mathrm{E}-09 \\
1.1 \mathrm{E}-08 \\
6.7 \mathrm{E}-09 \\
3.8 \mathrm{E}-08 \\
6.5 \mathrm{E}-09 \\
6.9 \mathrm{E}-05 \\
1.9 \mathrm{E}-06 \\
2.6 \mathrm{E}-06 \\
3.4 \mathrm{E}-05 \\
3.8 \mathrm{E}-07 \\
3.1 \mathrm{E}-05 \\
1.1 \mathrm{E}-08 \\
1.3 \mathrm{E}-05 \\
6.1 \mathrm{E}-09 \\
6.8 \mathrm{E}-05 \\
1.1 \mathrm{E}-09 \\
9.0 \mathrm{E}-09 \\
2.6 \mathrm{E}-08 \\
2.3 \mathrm{E}-03\end{array}$ & $\begin{array}{l}5.2 \mathrm{E}-02 \\
0.0 \mathrm{E}+00 \\
2.7 \mathrm{E}-06 \\
4.2 \mathrm{E}-04 \\
1.1 \mathrm{E}-04 \\
9.6 \mathrm{E}-05 \\
5.7 \mathrm{E}-04 \\
5.4 \mathrm{E}-01 \\
4.7 \mathrm{E}-04 \\
2.5 \mathrm{E}+01 \\
6.0 \mathrm{E}-02 \\
2.3 \mathrm{E}-02 \\
0.0 \mathrm{E}+00 \\
2.4 \mathrm{E}+00 \\
0.0 \mathrm{E}+00 \\
0.0 \mathrm{E}+00 \\
0.0 \mathrm{E}+00 \\
1.9 \mathrm{E}-05 \\
0.0 \mathrm{E}+00 \\
3.2 \mathrm{E}-01 \\
8.1 \mathrm{E}-06 \\
4.2 \mathrm{E}-02 \\
3.5 \mathrm{E}-02 \\
0.0 \mathrm{E}+00 \\
2.0 \mathrm{E}-02 \\
0.0 \mathrm{E}+00 \\
4.7 \mathrm{E}-03 \\
0.0 \mathrm{E}+00 \\
4.2 \mathrm{E}-02 \\
0.0 \mathrm{E}+00 \\
1.4 \mathrm{E}-06 \\
3.9 \mathrm{E}-06 \\
1.3 \mathrm{E}+00\end{array}$ & $\begin{array}{l}2.1 \mathrm{E}-02 \\
1.1 \mathrm{E}-02 \\
1.3 \mathrm{E}-03 \\
3.4 \mathrm{E}-03 \\
7.4 \mathrm{E}-02 \\
4.7 \mathrm{E}-01 \\
4.7 \mathrm{E}-01 \\
3.9 \mathrm{E}-01 \\
4.1 \mathrm{E}-01 \\
3.4 \mathrm{E}-01 \\
0.0 \mathrm{E}+00 \\
7.3 \mathrm{E}-03 \\
5.1 \mathrm{E}-02 \\
0.0 \mathrm{E}+00 \\
1.5 \mathrm{E}-02 \\
1.1 \mathrm{E}-02 \\
1.1 \mathrm{E}-02 \\
0.0 \mathrm{E}+00 \\
1.0 \mathrm{E}-02 \\
8.6 \mathrm{E}-03 \\
1.3 \mathrm{E}-02 \\
5.6 \mathrm{E}-05 \\
1.2 \mathrm{E}-02 \\
7.8 \mathrm{E}-01 \\
2.2 \mathrm{E}-02 \\
2.7 \mathrm{E}-02 \\
1.2 \mathrm{E}-02 \\
1.2 \mathrm{E}-02 \\
1.1 \mathrm{E}-02 \\
2.6 \mathrm{E}-03 \\
2.9 \mathrm{E}-02 \\
5.3 \mathrm{E}-02 \\
2.6 \mathrm{E}-01\end{array}$ & CPO & $\cdots$ \\
\hline $\begin{array}{ll}\mathrm{Zr}-95 & \text { old } \\
\mathrm{Sb}-125 & \text { old } \\
\mathrm{Pb}-210 & \text { old } \\
\mathrm{Ra}-226 & \text { old } \\
\mathrm{Th}-\mathrm{Nat} & \\
\mathrm{U}-\mathrm{Nat} & \\
\mathrm{Pu} 6 \% & \\
\mathrm{Pu} 6 \% & 10 \mathrm{yr} \\
U-232 & Y \\
U-233 & Y \\
U-234 & Y \\
U-235+D & Y \\
U-236 & Y \\
U-238+D & Y\end{array}$ & $\begin{array}{l}5.97 \mathrm{E}+03 \\
4.34 \mathrm{E}+03 \\
2.20 \mathrm{E}+00 \\
1.66 \mathrm{E}+00 \\
4.17 \mathrm{E}-02 \\
2.12 \mathrm{E}-01 \\
1.68 \mathrm{E}-01 \\
1.77 \mathrm{E}-01 \\
1.17 \mathrm{E}-01 \\
6.12 \mathrm{E}-01 \\
6.26 \mathrm{E}-01 \\
6.75 \mathrm{E}-01 \\
6.62 \mathrm{E}-01 \\
7.01 \mathrm{E}-01\end{array}$ & $\begin{array}{l}1.5 \mathrm{E}+01 \\
1.9 \mathrm{E}+00 \\
2.8 \mathrm{E}-06 \\
3.0 \mathrm{E}-03 \\
1.1 \mathrm{E}-04 \\
3.9 \mathrm{E}-04 \\
1.3 \mathrm{E}-07 \\
2.7 \mathrm{E}-07 \\
2.2 \mathrm{E}-08 \\
1.8 \mathrm{E}-07 \\
5.5 \mathrm{E}-08 \\
1.0 \mathrm{E}-04 \\
3.3 \mathrm{E}-08 \\
1.7 \mathrm{E}-05\end{array}$ & $\begin{array}{l}7.6 \mathrm{E}+02 \\
3.5 \mathrm{E}+02 \\
1.7 \mathrm{E}+00 \\
4.7 \mathrm{E}+00 \\
1.1 \mathrm{E}-01 \\
1.1 \mathrm{E}+00 \\
2.8 \mathrm{E}-06 \\
2.5 \mathrm{E}-06 \\
1.1 \mathrm{E}-05 \\
6.1 \mathrm{E}-06 \\
3.6 \mathrm{E}-05 \\
3.5 \mathrm{E}-02 \\
3.0 \mathrm{E}-05 \\
1.6 \mathrm{E}+00\end{array}$ & $\begin{array}{l}0.0 \mathrm{E}+00 \\
0.0 \mathrm{E}+00 \\
1.4 \mathrm{E}-01 \\
7.1 \mathrm{E}-01 \\
2.3 \mathrm{E}-02 \\
1.2 \mathrm{E}-01 \\
1.0 \mathrm{E}-02 \\
1.1 \mathrm{E}-02 \\
7.6 \mathrm{E}-03 \\
3.0 \mathrm{E}-02 \\
3.0 \mathrm{E}-02 \\
2.5 \mathrm{E}-02 \\
2.6 \mathrm{E}-02 \\
2.2 \mathrm{E}-02\end{array}$ & $\begin{array}{l}\text { CPI } \\
\text { CPD }\end{array}$ & $\begin{array}{l}\text { GMO } \\
\text { GMO }\end{array}$ \\
\hline
\end{tabular}


Table 3. Soil Limits and Instrument Response - $100 \mathrm{mrem} / \mathrm{y}$

\begin{tabular}{|c|c|c|c|c|c|c|}
\hline Nuclide & $\begin{array}{c}\text { Soil Limit } \\
\mathrm{pCj} / \mathrm{g}\end{array}$ & $\begin{array}{c}\text { Estimate } \\
\mathrm{CP} W / \mathrm{C} \\
(\mathrm{mR} / \mathrm{h})\end{array}$ & $\begin{array}{c}\text { [nstrumen } \\
\text { GM P-11 } \\
\text { (cpm) }\end{array}$ & $\frac{\text { Readings }}{\text { PAM }}$ & Dete & $\begin{array}{l}\text { How } \\
\text { ectable }\end{array}$ \\
\hline 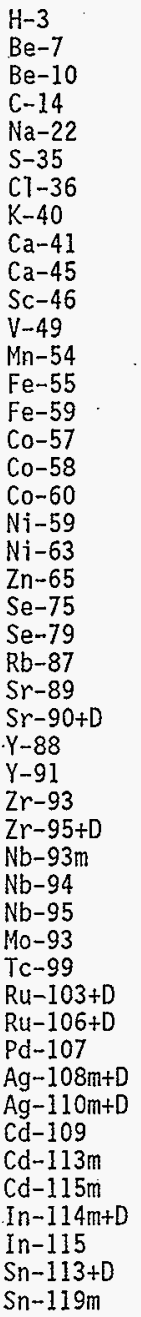 & $\begin{array}{l}3.74 E+07 \\
6.79 E+07 \\
2.29 E+04 \\
1.30 E+06 \\
3.01 E+05 \\
6.39 E+06 \\
2.95 E+05 \\
1.63 E+05 \\
2.08 E+06 \\
1.24 E+06 \\
6.16 E+05 \\
2.52 E+07 \\
9.75 E+05 \\
2.39 E+06 \\
1.65 E+06 \\
1.11 E+06 \\
1.67 E+06 \\
3.24 E+04 \\
4.73 E+06 \\
1.94 E+06 \\
2.65 E+05 \\
7.02 E+05 \\
2.98 E+05 \\
6.18 E+05 \\
1.63 E+06 \\
1.49 E+04 \\
5.38 E+05 \\
5.32 E+05 \\
2.56 E+04 \\
7.91 E+05 \\
2.81 E+05 \\
1.94 E+04 \\
5.39 E+06 \\
2.61 E+05 \\
7.33 E+05 \\
3.50 E+06 \\
2.15 E+04 \\
6.37 E+05 \\
2.79 E+04 \\
1.29 E+05 \\
7.63 E+04 \\
4.59 E+03 \\
4.48 E+05 \\
3.45 E+05 \\
2.05 E+03 \\
1.19 E+06 \\
1.36 E+05\end{array}$ & $\begin{array}{l}0.0 \mathrm{E}+00 \\
3.4 \mathrm{E}+03 \\
5.2 \mathrm{E}-03 \\
4.1 \mathrm{E}-03 \\
6.7 \mathrm{E}+02 \\
2.3 \mathrm{E}-02 \\
1.5 \mathrm{E}-01 \\
2.6 \mathrm{E}+01 \\
0.0 \mathrm{E}+00 \\
1.8 \mathrm{E}-02 \\
1.2 \mathrm{E}+03 \\
0.0 \mathrm{E}+00 \\
8.2 \mathrm{E}+02 \\
0.0 \mathrm{E}+00 \\
2.0 \mathrm{E}+03 \\
1.1 \mathrm{E}+02 \\
1.7 \mathrm{E}+03 \\
8.2 \mathrm{E}+01 \\
0.0 \mathrm{E}+00 \\
0.0 \mathrm{E}+00 \\
1.5 \mathrm{E}+02 \\
2.7 \mathrm{E}+02 \\
1.3 \mathrm{E}-03 \\
2.0 \mathrm{E}-02 \\
2.9 \mathrm{E}+00 \\
7.0 \mathrm{E}-02 \\
1.5 \mathrm{E}+03 \\
3.0 \mathrm{E}+00 \\
0.0 \mathrm{E}+00 \\
5.9 \mathrm{E}+02 \\
8.9 \mathrm{E}-03 \\
3.1 \mathrm{E}+01 \\
4.2 \mathrm{E}+03 \\
4.7 \mathrm{E}-02 \\
2.1 \mathrm{E}-02 \\
1.7 \mathrm{E}+03 \\
4.8 \mathrm{E}+00 \\
0.0 \mathrm{E}+00 \\
4.6 \mathrm{E}+01 \\
3.6 \mathrm{E}+02 \\
3.2 \mathrm{E}-01 \\
6.3 \mathrm{E}-04 \\
1.1 \mathrm{E}+01 \\
3.1 \mathrm{E}+01 \\
1.8 \mathrm{E}-04 \\
3.2 \mathrm{E}+02 \\
1.1 \mathrm{E}+00\end{array}$ & $\begin{array}{l}0.0 \mathrm{E}+00 \\
0.0 \mathrm{E}+00 \\
6.2 \mathrm{E}+03 \\
1.1 \mathrm{E}+04 \\
8.1 \mathrm{E}+04 \\
6.2 \mathrm{E}+04 \\
1.2 \mathrm{E}+05 \\
1.6 \mathrm{E}+05 \\
0.0 \mathrm{E}+00 \\
5.1 \mathrm{E}+04 \\
5.8 \mathrm{E}+04 \\
0.0 \mathrm{E}+00 \\
0.0 \mathrm{E}+00 \\
0.0 \mathrm{E}+00 \\
1.9 \mathrm{E}+05 \\
3.1 \mathrm{E}+03 \\
6.4 \mathrm{E}+04 \\
2.2 \mathrm{E}+03 \\
0.0 \mathrm{E}+00 \\
0.0 \mathrm{E}+00 \\
5.1 \mathrm{E}+02 \\
3.1 \mathrm{E}+03 \\
1.5 \mathrm{E}+03 \\
2.7 \mathrm{E}+04 \\
2.2 \mathrm{E}+06 \\
4.1 \mathrm{E}+04 \\
6.8 \mathrm{E}+02 \\
7.4 \mathrm{E}+05 \\
0.0 \mathrm{E}+00 \\
8.5 \mathrm{E}+04 \\
0.0 \mathrm{E}+00 \\
3.2 \mathrm{E}+03 \\
5.0 \mathrm{E}+04 \\
0.0 \mathrm{E}+00 \\
3.9 \mathrm{E}+04 \\
1.4 \mathrm{E}+05 \\
9.3 \mathrm{E}+04 \\
0.0 \mathrm{E}+00 \\
4.2 \mathrm{E}+03 \\
1.4 \mathrm{E}+04 \\
0.0 \mathrm{E}+00 \\
1.1 \mathrm{E}+03 \\
6.5 \mathrm{E}+05 \\
7.1 \mathrm{E}+05 \\
3.6 \mathrm{E}+02 \\
2.7 \mathrm{E}+05 \\
0.0 \mathrm{E}+00\end{array}$ & $\begin{array}{l}0.0 E+00 \\
0.0 E+00 \\
0.0 E+00 \\
0.0 E+00 \\
0.0 E+00 \\
0.0 E+00 \\
0.0 E+00 \\
0.0 E+00 \\
0.0 E+00 \\
0.0 E+00 \\
0.0 E+00 \\
0.0 E+00 \\
0.0 E+00 \\
0.0 E+00 \\
0.0 E+00 \\
0.0 E+00 \\
0.0 E+00 \\
0.0 E+00 \\
0.0 E+00 \\
0.0 E+00 \\
0.0 E+00 \\
0.0 E+00 \\
0.0 E+00 \\
0.0 E+00 \\
0.0 E+00 \\
0.0 E+00 \\
0.0 E+00 \\
0.0 E+00 \\
0.0 E+00 \\
0.0 E+00 \\
0.0 E+00 \\
0.0 E+00 \\
0.0 E+00 \\
0.0 E+00 \\
0.0 E+00 \\
0.0 E+00 \\
0.0 E+00 \\
0.0 E+00 \\
0.0 E+00 \\
0.0 E+00 \\
0.0 E+00 \\
0.0 E+00 \\
0.0 E+00 \\
0.0 E+00 \\
0.0 E+00 \\
0.0 E+00 \\
0.0 E+00\end{array}$ & $\begin{array}{l}\text { CP1 } \\
\text { CP3 } \\
\text { CP2 } \\
\text { CP3 } \\
\text { CP2 } \\
\text { CP3 } \\
\text { CP1 } \\
\text { CP2 } \\
\text { CP2 } \\
\text { CP0 } \\
\text { CP3 } \\
\text { CP0 } \\
\text { CP2 } \\
\text { CP1 } \\
\text { CP3 }\end{array}$ & $\begin{array}{l}\text { GM1 } \\
\text { GM2 } \\
\text { GM2 } \\
\text { GM2 } \\
\text { GM3 } \\
\text { GM3 } \\
\text { GM2 } \\
\text { GM2 } \\
\\
\text { GM3 } \\
\text { GM1 } \\
\text { GM2 } \\
\text { GM1 } \\
\text { GMO } \\
\text { GM1 } \\
\text { GM1 } \\
\text { GM2 } \\
\text { GM4 } \\
\text { GM2 } \\
\text { GMO } \\
\text { GM3 } \\
\text { GM2 }\end{array}$ \\
\hline
\end{tabular}


Table 3. Soi1 Limits and Instrument Response - $100 \mathrm{mrem} / \mathrm{y}$, Continued

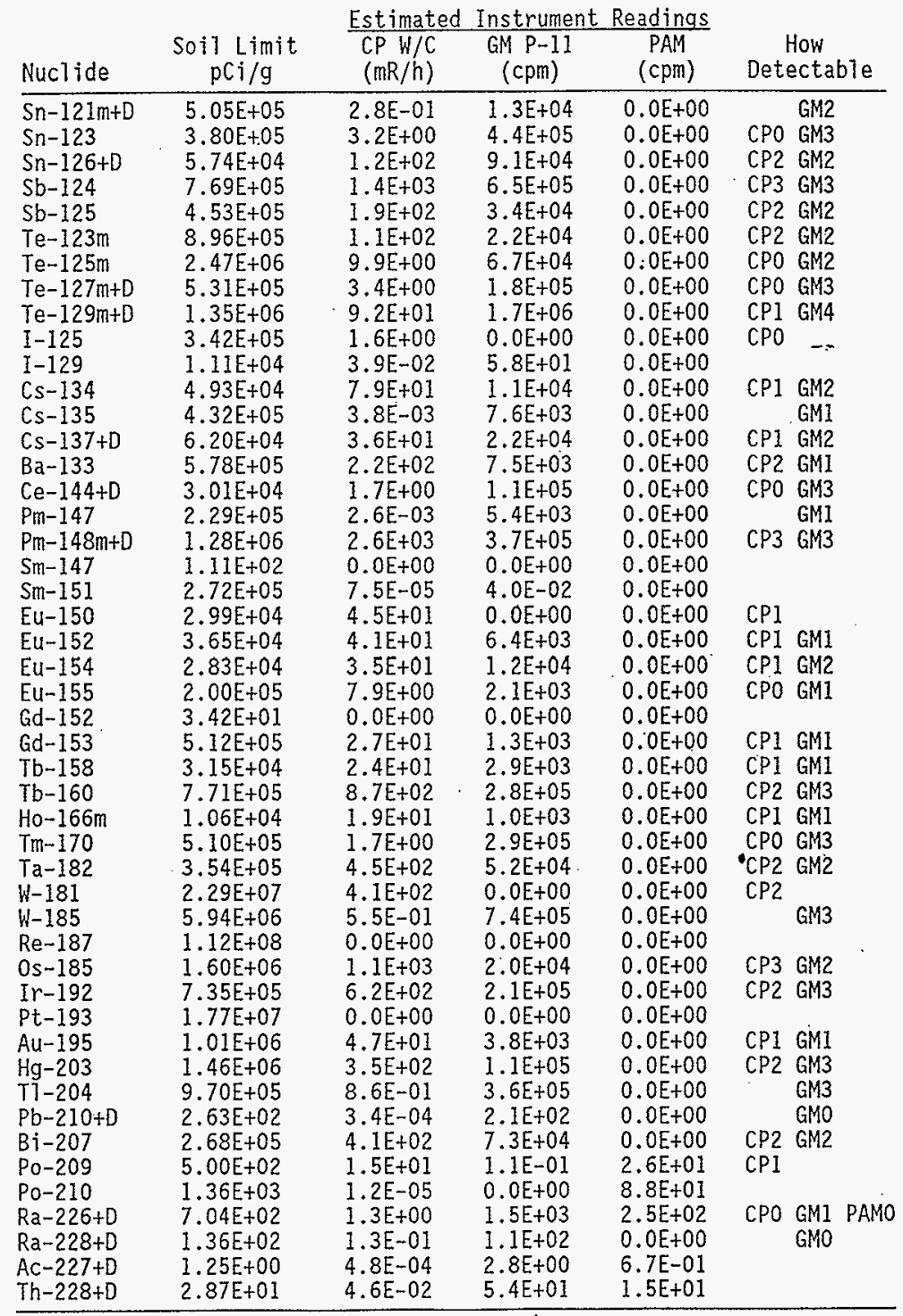


Table 3. Soil Limits and Instrument Response - $100 \mathrm{mrem} / \mathrm{y}$, Continued

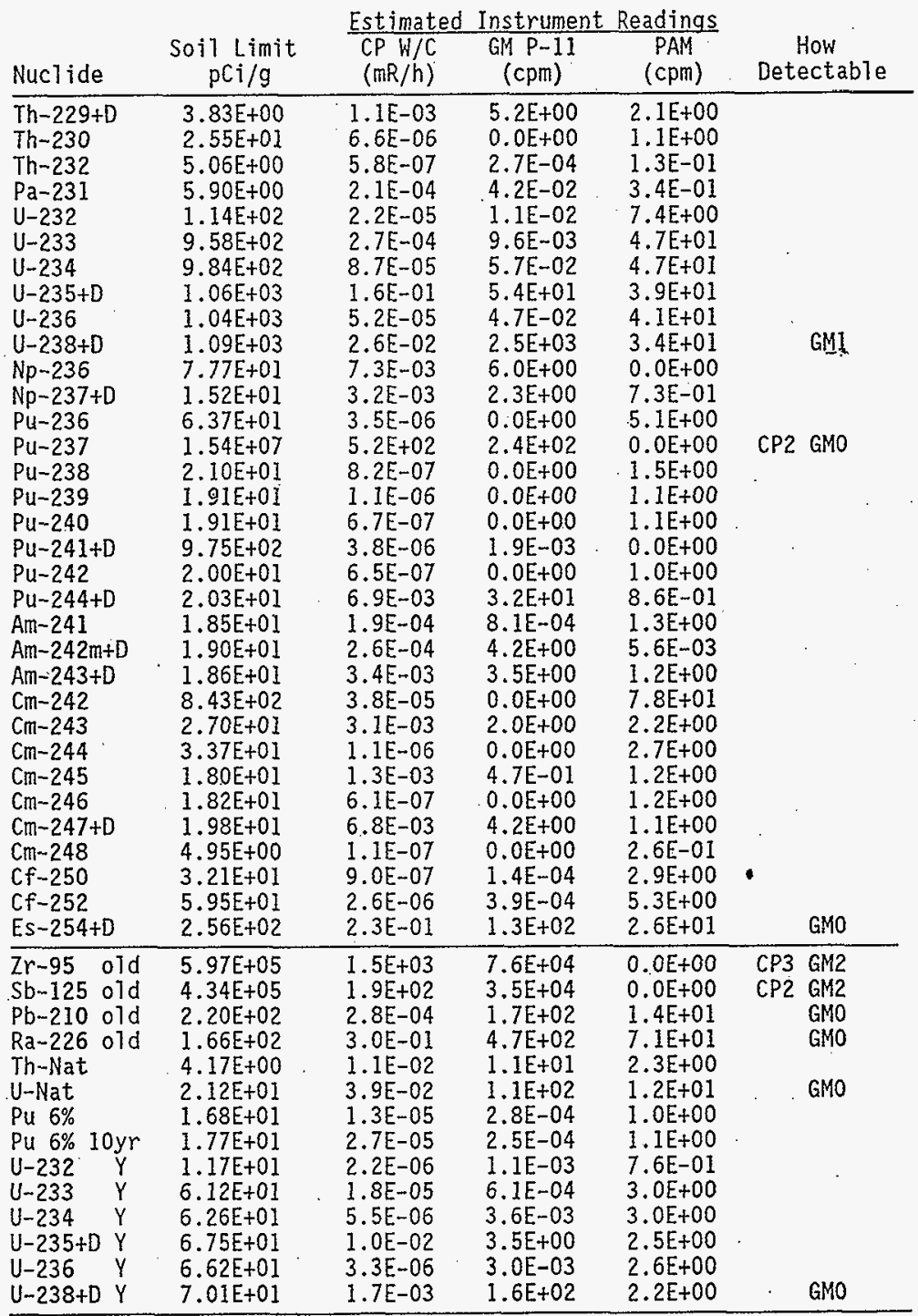


Table 4. Soil Limits and Instrument Response- $500 \mathrm{mrem} / \mathrm{y}$

\begin{tabular}{|c|c|c|c|c|c|c|}
\hline Nuclide & $\begin{array}{c}\text { Soil Limit } \\
\mathrm{pCi} / \mathrm{g}\end{array}$ & $\frac{\text { Estimate }}{C P W / C}$ & $\frac{\text { Instrumen }}{G M P-11}$ & 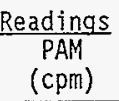 & Dete & $\begin{array}{l}\text { How } \\
\text { ectable }\end{array}$ \\
\hline $\begin{array}{l}H-3 \\
B e-7 \\
B e-10 \\
C-14 \\
\mathrm{Na}-22 \\
\mathrm{~S}-35 \\
\mathrm{C} 1-36 \\
\mathrm{~K}-40 \\
\mathrm{Ca}-41 \\
\mathrm{Ca}-45 \\
\mathrm{SC}-46 \\
\mathrm{~V}-49 \\
\mathrm{Mn}-54 \\
\mathrm{Fe}-55 \\
\mathrm{Fe}-59 \\
\mathrm{CO}-57 \\
\mathrm{Co}-58 \\
\mathrm{CO}-60 \\
\mathrm{Nj}-59 \\
\mathrm{Ni}-63 \\
\mathrm{Zn}-65 \\
\mathrm{Se}-75 \\
\mathrm{Se}-79 \\
\mathrm{Rb}-87 \\
\mathrm{Sr}-89 \\
\mathrm{Sr}-90+\mathrm{D} \\
\mathrm{Y}-88 \\
\mathrm{Y}-91 \\
\mathrm{Zr}-93 \\
\mathrm{Zr}-95+\mathrm{D} \\
\mathrm{Nb}-93 \mathrm{~m} \\
\mathrm{Nb}-94 \\
\mathrm{Nb}-95 \\
\mathrm{Mo}-93 \\
\mathrm{TC}-99 \\
\mathrm{Ru}-103+\mathrm{D} \\
\mathrm{Ru}-106+\mathrm{D} \\
\mathrm{Pd}-107 \\
\mathrm{Ag}-108 \mathrm{~m}+\mathrm{D} \\
\mathrm{Ag}-110 \mathrm{~m}+\mathrm{D} \\
\mathrm{Cd}-109 \\
\mathrm{Cd}-113 \mathrm{~m} \\
\mathrm{Cd}-115 \mathrm{~m} \\
\mathrm{Sn}-119 \mathrm{~m}\end{array}$ & $\begin{array}{l}1.87 E+08 \\
3.40 E+08 \\
1.14 E+05 \\
6.48 E+06 \\
1.50 E+06 \\
3.19 E+07 \\
1.48 E+06 \\
8.16 E+05 \\
1.04 E+07 \\
6.21 E+06 \\
3.08 E+06 \\
1.26 E+08 \\
4.88 E+06 \\
1.20 E+07 \\
8.25 E+06 \\
5.56 E+06 \\
8.36 E+06 \\
1.62 E+05 \\
2.36 E+07 \\
9.71 E+06 \\
1.33 E+06 \\
3.51 E+06 \\
1.49 E+06 \\
3.09 E+06 \\
8.14 E+06 \\
7.44 E+04 \\
2.69 E+06 \\
2.66 E+06 \\
1.28 E+05 \\
3.96 E+06 \\
1.41 E+06 \\
9.71 E+04 \\
2.69 E+07 \\
1.30 E+06 \\
3.66 E+06 \\
1.75 E+07 \\
1.08 E+05 \\
3.19 E+06 \\
1.40 E+05 \\
6.45 E+05 \\
3.82 E+05 \\
2.29 E+04 \\
2.24 E+06 \\
1.72 E+06 \\
1.02 E+04 \\
5.95 E+06 \\
6.80 E+06\end{array}$ & $\begin{array}{l}0.0 \mathrm{E}+00 \\
1.7 \mathrm{E}+04 \\
2.6 \mathrm{E}-02 \\
2.1 \mathrm{E}-02 \\
3.3 \mathrm{E}+03 \\
1.1 \mathrm{E}-01 \\
7.3 \mathrm{E}-01 \\
1.3 \mathrm{E}+02 \\
0.0 \mathrm{E}+00 \\
8.8 \mathrm{E}-02 \\
6.2 \mathrm{E}+03 \\
0.0 \mathrm{E}+00 \\
4.1 \mathrm{E}+03 \\
0.0 \mathrm{E}+00 \\
9.8 \mathrm{E}+03 \\
5.7 \mathrm{E}+02 \\
8.3 \mathrm{E}+03 \\
4.1 \mathrm{E}+02 \\
0.0 \mathrm{E}+00 \\
0.0 \mathrm{E}+00 \\
7.7 \mathrm{E}+02 \\
1.3 \mathrm{E}+03 \\
6.7 \mathrm{E}-03 \\
9.8 \mathrm{E}-02 \\
1.4 \mathrm{E}+01 \\
3.5 \mathrm{E}-01 \\
7.3 \mathrm{E}+03 \\
1.5 \mathrm{E}+01 \\
0.0 \mathrm{E}+00 \\
3.0 \mathrm{E}+03 \\
4.4 \mathrm{E}-02 \\
1.6 \mathrm{E}+02 \\
2.1 \mathrm{E}+04 \\
2.3 \mathrm{E}-01 \\
1.0 \mathrm{E}-01 \\
8.4 \mathrm{E}+03 \\
2.4 \mathrm{E}+01 \\
0.0 \mathrm{E}+00 \\
2.3 \mathrm{E}+02 \\
1.8 \mathrm{E}+03 \\
1.6 \mathrm{E}+00 \\
3.1 \mathrm{E}-03 \\
5.5 \mathrm{E}+01 \\
1.5 \mathrm{E}+02 \\
8.8 \mathrm{E}-04 \\
1.6 \mathrm{E}+03 \\
5.7 \mathrm{E}+00\end{array}$ & $\begin{array}{l}0.0 E+00 \\
0.0 E+00 \\
3.1 E+04 \\
5.4 E+04 \\
4.0 E+05 \\
3.1 E+05 \\
5.8 E+05 \\
8.2 E+05 \\
0.0 E+00 \\
2.5 E+05 \\
2.9 E+05 \\
0.0 E+00 \\
0.0 E+00 \\
0.0 E+00 \\
9.3 E+05 \\
1.6 E+04 \\
3.2 E+05 \\
1.1 E+04 \\
0.0 E+00 \\
0.0 E+00 \\
2.5 E+03 \\
1.5 E+04 \\
7.3 E+03 \\
1.4 E+05 \\
1.1 E+07 \\
2.1 E+05 \\
3.4 E+03 \\
3.7 E+06 \\
0.0 E+00 \\
4.2 E+05 \\
0.0 E+00 \\
1.6 E+04 \\
2.5 E+05 \\
0.0 E+00 \\
1.9 E+05 \\
6.9 E+05 \\
4.6 E+05 \\
0.0 E+00 \\
2.1 E+04 \\
7.2 E+04 \\
0.0 E+00 \\
5.6 E+03 \\
3.3 E+06 \\
3.6 E+06 \\
1.8 E+03 \\
1.4 E+06 \\
0.0 E+00\end{array}$ & $\begin{array}{l}0.0 E+00 \\
0.0 E+00 \\
0.0 E+00 \\
0.0 E+00 \\
0.0 E+00 \\
0.0 E+00 \\
0.0 E+00 \\
0.0 E+00 \\
0.0 E+00 \\
0.0 E+00 \\
0.0 E+00 \\
0.0 E+00 \\
0.0 E+00 \\
0.0 E+00 \\
0.0 E+00 \\
0.0 E+00 \\
0.0 E+00 \\
0.0 E+00 \\
0.0 E+00 \\
0.0 E+00 \\
0.0 E+00 \\
0.0 E+00 \\
0.0 E+00 \\
0.0 E+00 \\
0.0 E+00 \\
0.0 E+00 \\
0.0 E+00 \\
0.0 E+00 \\
0.0 E+00 \\
0.0 E+00 \\
0.0 E+00 \\
0.0 E+00 \\
0.0 E+00 \\
0.0 E+00 \\
0.0 E+00 \\
0.0 E+00 \\
0.0 E+00 \\
0.0 E+00 \\
0.0 E+00 \\
0.0 E+00 \\
0.0 E+00 \\
0.0 E+00 \\
0.0 E+00 \\
0.0 E+00 \\
0.0 E+00 \\
0.0 E+00 \\
0.0 E+00\end{array}$ & $\begin{array}{l}\text { CP1 } \\
\text { CP3 } \\
\text { CP1 } \\
\text { CP3 } \\
\text { CP2 } \\
\text { CP4 } \\
\text { CP3 } \\
\text { CP1 }\end{array}$ & $\begin{array}{l}\text { GM1 } \\
\text { GM2 } \\
\text { GM1 } \\
\text { GM3 } \\
\text { GM5 } \\
\text { GM3 } \\
\text { GM1 } \\
\text { GM4 } \\
\text { GM3 } \\
\text { GM2 } \\
\text { GM3 } \\
\text { GM3 } \\
\text { GM3 } \\
\text { GM3 }\end{array}$ \\
\hline
\end{tabular}


Table 4. Soil Limits and Instrument Response - $500 \mathrm{mrem} / \mathrm{y}$, Continued

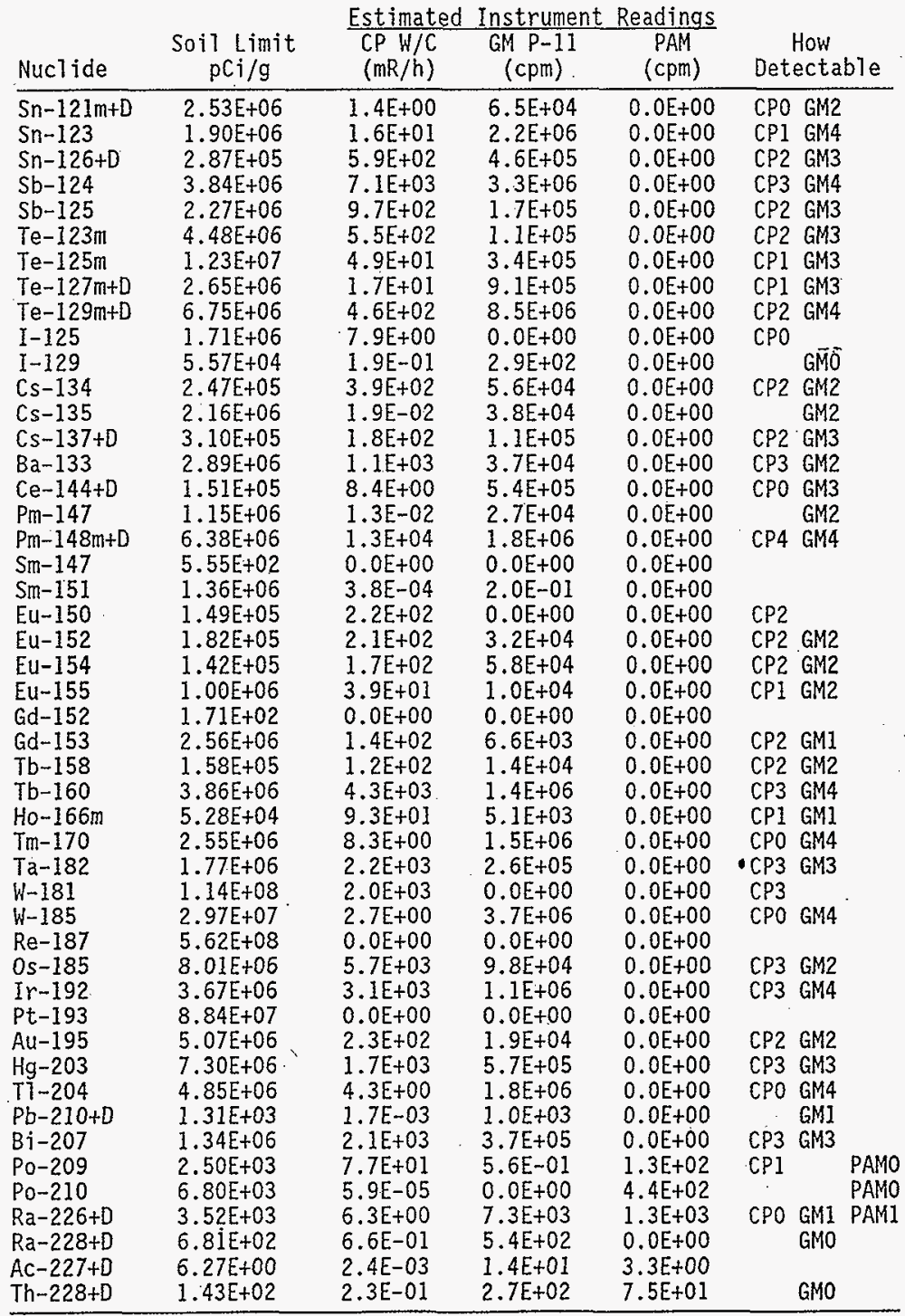


Table 4. Soil Limits and Instrument Response $-500 \mathrm{mrem} / \mathrm{y}$, Continued

\begin{tabular}{|c|c|c|c|c|c|c|c|}
\hline Nuclide & $\begin{array}{c}\text { Soil Limit } \\
\mathrm{pCi} / \mathrm{g}\end{array}$ & $\begin{array}{l}\text { Estimate } \\
\mathrm{CPW} / \mathrm{C} \\
(\mathrm{mR} / \mathrm{h})\end{array}$ & $\begin{array}{c}\text { Instrumer } \\
\text { GM P-11 } \\
\text { (cpm) }\end{array}$ & 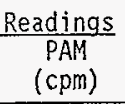 & & $\begin{array}{l}\text { How } \\
\text { ecta }\end{array}$ & \\
\hline $\begin{array}{l}T h-229+D \\
T h-230 \\
T h-232 \\
P a-231 \\
U-232 \\
U-233 \\
U-234 \\
U-235+D \\
U-236 \\
U-238+D \\
N p-236 \\
N p-237+D \\
P u-236 \\
P u-237 \\
P u-238 \\
P u-239 \\
P u-240 \\
P u-241+D \\
P u-242 \\
P u-244+D \\
A m-241 \\
A m-242 m+D \\
A m-243+D \\
C m-242 \\
C m-243 \\
C m-244 \\
C m-245 \\
C m-246 \\
C m-247+D \\
C m-248 \\
C f-250 \\
C f-252 \\
E s-254+D\end{array}$ & $\begin{array}{l}1.92 E+01 \\
1.28 E+02 \\
2.53 E+01 \\
2.95 E+01 \\
5.69 E+02 \\
4.79 E+03 \\
4.92 E+03 \\
5.30 E+03 \\
5.21 E+03 \\
5.47 E+03 \\
3.88 E+02 \\
7.58 E+01 \\
3.18 E+02 \\
7.70 E+07 \\
1.05 E+02 \\
9.54 E+01 \\
9.54 E+01 \\
4.87 E+03 \\
9.98 E+01 \\
1.02 E+02 \\
9.23 E+01 \\
9.48 E+01 \\
9.30 E+01 \\
4.21 E+03 \\
1.35 E+02 \\
1.68 E+02 \\
9.00 E+01 \\
9.08 E+01 \\
9.88 E+01 \\
2.48 E+01 \\
1.51 E+02 \\
2.98 E+02 \\
1.28 E+03\end{array}$ & $\begin{array}{l}5.7 \mathrm{E}-03 \\
3.3 \mathrm{E}-05 \\
2.9 \mathrm{E}-06 \\
1.1 \mathrm{E}-03 \\
1.1 \mathrm{E}-04 \\
1.4 \mathrm{E}-03 \\
4.4 \mathrm{E}-04 \\
7.9 \mathrm{E}-01 \\
2.6 \mathrm{E}-04 \\
1.3 \mathrm{E}-01 \\
3.7 \mathrm{E}-02 \\
1.6 \mathrm{E}-02 \\
1.8 \mathrm{E}-05 \\
2.6 \mathrm{E}+03 \\
4.1 \mathrm{E}-06 \\
5.6 \mathrm{E}-06 \\
3.4 \mathrm{E}-06 \\
1.9 \mathrm{E}-05 \\
3.3 \mathrm{E}-06 \\
3.5 \mathrm{E}-02 \\
9.5 \mathrm{E}-04 \\
1.3 \mathrm{E}-03 \\
1.7 \mathrm{E}-02 \\
1.9 \mathrm{E}-04 \\
1.6 \mathrm{E}-02 \\
5.6 \mathrm{E}-06 \\
6.3 \mathrm{E}-03 \\
3.0 \mathrm{E}-06 \\
3.4 \mathrm{E}-02 \\
5.7 \mathrm{E}-07 \\
4.5 \mathrm{E}-06 \\
1.3 \mathrm{E}-05 \\
1.1 \mathrm{E}+00\end{array}$ & $\begin{array}{l}2.6 \mathrm{E}+01 \\
0.0 \mathrm{E}+00 \\
1.4 \mathrm{E}-03 \\
2.1 \mathrm{E}-01 \\
5.5 \mathrm{E}-02 \\
4.8 \mathrm{E}-02 \\
2.9 \mathrm{E}-01 \\
2.7 \mathrm{E}+02 \\
2.3 \mathrm{E}-01 \\
1.2 \mathrm{E}+04 \\
3.0 \mathrm{E}+01 \\
1.2 \mathrm{E}+01 \\
0.0 \mathrm{E}+00 \\
1.2 \mathrm{E}+03 \\
0.0 \mathrm{E}+00 \\
0.0 \mathrm{E}+00 \\
0.0 \mathrm{E}+00 \\
9.3 \mathrm{E}-03 \\
0.0 \mathrm{E}+00 \\
1.6 \mathrm{E}+02 \\
4.0 \mathrm{E}-03 \\
2.1 \mathrm{E}+01 \\
1.7 \mathrm{E}+01 \\
0.0 \mathrm{E}+00 \\
1.0 \mathrm{E}+01 \\
0.0 \mathrm{E}+00 \\
2.4 \mathrm{E}+00 \\
0.0 \mathrm{E}+00 \\
2.1 \mathrm{E}+01 \\
0.0 \mathrm{E}+00 \\
7.2 \mathrm{E}-04 \\
1.9 \mathrm{E}-03 \\
6.4 \mathrm{E}+02\end{array}$ & $\begin{array}{l}1.0 \mathrm{E}+01 \\
5.7 \mathrm{E}+00 \\
6.5 \mathrm{E}-01 \\
1.7 \mathrm{E}+00 \\
3.7 \mathrm{E}+01 \\
2.4 \mathrm{E}+02 \\
2.4 \mathrm{E}+02 \\
1.9 \mathrm{E}+02 \\
2.0 \mathrm{E}+02 \\
1.7 \mathrm{E}+02 \\
0.0 \mathrm{E}+00 \\
3.6 \mathrm{E}+00 \\
2.5 \mathrm{E}+01 \\
0.0 \mathrm{E}+00 \\
7.4 \mathrm{E}+00 \\
5.7 \mathrm{E}+00 \\
5.7 \mathrm{E}+00 \\
0.0 \mathrm{E}+00 \\
5.2 \mathrm{E}+00 \\
4.3 \mathrm{E}+00 \\
6.5 \mathrm{E}+00 \\
2.8 \mathrm{E}-02 \\
5.9 \mathrm{E}+00 \\
3.9 \mathrm{E}+02 \\
1.1 \mathrm{E}+01 \\
1.4 \mathrm{E}+01 \\
6.0 \mathrm{E}+00 \\
5.1 \mathrm{E}+00 \\
5.3 \mathrm{E}+00 \\
1.3 \mathrm{E}+00 \\
1.4 \mathrm{E}+01 \\
2.7 \mathrm{E}+01 \\
1.3 \mathrm{E}+02\end{array}$ & CPO & $\begin{array}{l}\text { GMO } \\
\text { GM? }\end{array}$ & $\begin{array}{l}\text { PAMO } \\
\text { PAMO } \\
\text { PAMO } \\
\text { PAMO } \\
\text { PAMO }\end{array}$ \\
\hline $\begin{array}{ll}\mathrm{Zr}-95 & \text { old } \\
\mathrm{Sb}-125 & \text { old } \\
\mathrm{Pb}-210 & \text { old } \\
\mathrm{Ra}-226 & \text { old } \\
\text { Th-Nat } & \\
\mathrm{U}-\mathrm{Nat} & \\
\mathrm{Pu} 6 \% & \\
\mathrm{Pu} 6 \% & 10 \mathrm{yr} \\
\mathrm{U}-232 & \mathrm{Y} \\
\mathrm{U}-233 & \mathrm{Y} \\
\mathrm{U}-234 & \mathrm{Y} \\
\mathrm{U}-235+\mathrm{D} & \mathrm{Y} \\
\mathrm{U}-236 & \mathrm{Y} \\
\mathrm{U}-238+D & Y\end{array}$ & $\begin{array}{l}2.99 \mathrm{E}+06 \\
2.17 \mathrm{E}+06 \\
1.10 \mathrm{E}+03 \\
8.30 \mathrm{E}+02 \\
2.08 \mathrm{E}+01 \\
1.06 \mathrm{E}+02 \\
8.41 \mathrm{E}+01 \\
8.84 \mathrm{E}+01 \\
5.84 \mathrm{E}+01 \\
3.06 \mathrm{E}+02 \\
3.13 \mathrm{E}+02 \\
3.38 \mathrm{E}+02 \\
3.31 \mathrm{E}+02 \\
3.50 \mathrm{E}+02\end{array}$ & $\begin{array}{l}7.4 \mathrm{E}+03 \\
9.3 \mathrm{E}+02 \\
1.4 \mathrm{E}-03 \\
1.5 \mathrm{E}+00 \\
5.3 \mathrm{E}-02 \\
1.9 \mathrm{E}-01 \\
6.3 \mathrm{E}-05 \\
1.4 \mathrm{E}-04 \\
1.1 \mathrm{E}-05 \\
8.8 \mathrm{E}-05 \\
2.8 \mathrm{E}-05 \\
5.0 \mathrm{E}-02 \\
1.6 \mathrm{E}-05 \\
8.4 \mathrm{E}-03\end{array}$ & $\begin{array}{l}3.8 \mathrm{E}+05 \\
1.8 \mathrm{E}+05 \\
8.6 \mathrm{E}+02 \\
2.4 \mathrm{E}+03 \\
5.6 \mathrm{E}+01 \\
5.4 \mathrm{E}+02 \\
1.4 \mathrm{E}-03 \\
1.2 \mathrm{E}-03 \\
5.6 \mathrm{E}-03 \\
3.1 \mathrm{E}-03 \\
1.8 \mathrm{E}-02 \\
1.7 \mathrm{E}+01 \\
1.5 \mathrm{E}-02 \\
7.8 \mathrm{E}+02\end{array}$ & $\begin{array}{l}0.0 E+00 \\
0.0 E+00 \\
7.2 E+01 \\
3.5 E+02 \\
1.1 E+01 \\
5.8 E+01 \\
5.1 E+00 \\
5.5 E+00 \\
3.8 E+00 \\
1.5 E+01 \\
1.5 E+01 \\
1.2 E+01 \\
1.3 E+01 \\
1.1 E+01\end{array}$ & $\begin{array}{l}\text { CP3 } \\
\text { CP2 } \\
\text { CPO }\end{array}$ & $\begin{array}{l}\text { GM3 } \\
\text { GM3 } \\
\text { GMO } \\
\text { GM1 } \\
\text { GMO }\end{array}$ & PAMO \\
\hline
\end{tabular}




\subsection{List of References}

Carbaugh, E. H. and D. E. Bihl, "Bioassay Criteria for Environmental Restoration Workers", pp 457-466 in Environmental Health Physics, Columbia Chapter, Health Physics Society, 1993.

International Commission on Radiological Protection (ICRP), Report of the Task Group on Reference Man, ICRP Publication 23, Pergamon Press, New York, New York, 1975.

International Commission on Radiological Protection (ICRP), Recommendations of the International Commission on Radiological Protection, ICRP Publication 26, Pergamon Press, New York, New York, 1977.

Kocher, D. C., Radioactive Decay Data Tab7es, (DOE/TIC-11026), U.S. Government Printing office, 1981 .

National Bureau of Standards, Applied Mathematics Series, Tables for the Analysis of Beta Spectra, U.S. Government Printing Office, 1952.

Rittmann, P. D., ISO-PC Version 1.98 User's Guide; WHC-SD-WM-UM-030 Rev 0, 1995.

Siegbahn, K., Ed., A7pha-, Beta-, and Gamma-Ray Spectroscopy, North-Holland Co., New York, 1968.

U.S. Department of Health, Education, and Welfare, Radiological Health Handbook, U.S. Government Printing office, 1970 .

U.S. Environmental Protection Agency Federal Guidance Report Number 11, Limiting Values of Radionuclide Intake and Air Concentration and Dose Conversion Factors for Inhalation, Submersion, and Ingestion, (EPA-520/1-88-020), 1988.

U.S. Environmental Protection Agency Federal Guidance Report Number 12, External Exposure to Radionuclides in Air, Water, and Soil, (EPA 402-R-93-081), 1993. 
HNF-2418

Appendix A. Data and Formulas Used in the Calculations 


\section{RADIOACTIVE DECAY METHOD}

A spreadsheet was used for these calculations. The specific decay chains used by the spreadsheet are shown in Table A-1 below. The longest decay chain considered has 5 members. Some chains end even though there are significant members further down the chain. An example of this approximation is the Am-241, which ends at U-233 instead of including Th-229. Due to the long half-lives of the omitted nuclides there will be little ingrowth of these progeny for decay times less than 1000 years. Hence they can be omitted.

The " $+D$ " designator means that there are short half-live progeny nuclides produced by the decay of this nuclide. Table A-2 1 ists the short-lived progeny which were lumped with the parent nuclide. These are sometimes called "impiicit daughters". The numbers in parentheses are the effective branching ratios for that nuclide when it is in secular equilibrium with its parent nuclide. The dose factors for all nuclides with implicit daughters were computed by adding the appropriate dose factors, weighted by the equilibrium branching ratios.

The parent nuclides shown on the leftmost column of Table A-1 generate additional nuclides which also contribute to the annual dose. At the start of the year of exposure the concentration of the Table A-1 parent nuclides has a unit value. The progeny nuclides shown on Table A-1 have zero activity. Note that the Table A-2 progeny are present in equilibrium amounts even at the beginning of the exposure scenario due to their short half-lives. As the year progresses, the parent nuclide concentration decreases according to the halfTife of that nuclide. In addition, the progeny nuclides shown in Table A-1 begin to accumulate in the soil.

Radioactive decay of the source as well as the ingrowth of any progeny nuclides is computed using the ordinary decay chain equations. The actual decay chains computed by the spreadsheet are shown in Table A-1 below. The decay equations shown below describe the decay of nuclide I (the parent) to nuclide 2 , then nuclide 3 , and finally nuclide 4 .

$$
\begin{aligned}
& A_{1}=A_{1}^{0} \operatorname{Exp}\left(-\lambda_{1} T\right) \\
& A_{2}=A_{2}^{0} \operatorname{Exp}\left(-\lambda_{2} T\right)+A_{1}^{0} \lambda_{2}\left\{\operatorname{Exp}\left(-\lambda_{1} T\right) /\left(\lambda_{2}-\lambda_{1}\right)+\operatorname{Exp}\left(-\lambda_{2} T\right) /\left(\lambda_{1}-\lambda_{2}\right)\right\} \\
& A_{3}=A_{3}^{0} \operatorname{Exp}\left(-\lambda_{3} T\right)+A_{2}^{0} \lambda_{3}\left\{\operatorname{Exp}\left(-\lambda_{2} T\right) /\left(\lambda_{3}-\lambda_{2}\right)+\operatorname{Exp}\left(-\lambda_{3} T\right) /\left(\lambda_{2}-\lambda_{3}\right)\right\} \\
& +A_{1}^{0} \lambda_{2} \lambda_{3} \mid \operatorname{Exp}\left(-\lambda_{1} T\right) /\left(\dot{\lambda}_{2}-\lambda_{1}\right) /\left(\lambda_{3}-\lambda_{1}\right)+\operatorname{Exp}\left(-\lambda_{2} T\right) /\left(\lambda_{1}-\lambda_{2}\right) /\left(\lambda_{3}-\lambda_{2}\right) \\
& \left.+\operatorname{Exp}\left(-\lambda_{3} T\right) /\left(\lambda_{1}-\lambda_{3}\right) /\left(\lambda_{2}-\lambda_{3}\right)\right\} \\
& A_{4}=A_{4}^{0} \operatorname{Exp}\left(-\lambda_{4} T\right)+A_{3}^{0} \lambda_{4}\left\{\operatorname{Exp}\left(-\lambda_{3} T\right) /\left(\lambda_{4}-\lambda_{3}\right)+\operatorname{Exp}\left(-\lambda_{4} T\right) /\left(\lambda_{3}-\lambda_{4}\right)\right\} \\
& +A_{2}^{0} \lambda_{3} \lambda_{4}\left\{\operatorname{Exp}\left(-\lambda_{2} T\right) /\left(\lambda_{3}-\lambda_{2}\right) /\left(\lambda_{4}-\lambda_{2}\right)+\operatorname{Exp}\left(-\lambda_{3} T\right) /\left(\lambda_{2}-\lambda_{3}\right) /\left(\lambda_{4}-\lambda_{3}\right)\right. \\
& \left.+\operatorname{Exp}\left(-\lambda_{4} T\right) /\left(\lambda_{2}-\lambda_{4}\right) /\left(\lambda_{3}-\lambda_{4}\right)\right\} \\
& +A_{1}^{0} \lambda_{2} \lambda_{3} \lambda_{4}\left\{\operatorname{Exp}\left(-\lambda_{1} T\right) /\left(\lambda_{2}-\lambda_{1}\right) /\left(\lambda_{3}-\lambda_{1}\right) /\left(\lambda_{4}-\lambda_{1}\right)\right. \\
& +\operatorname{Exp}\left(-\lambda_{2} T\right) /\left(\lambda_{1}-\lambda_{2}\right) /\left(\lambda_{3}-\lambda_{2}\right) /\left(\lambda_{4}-\lambda_{2}\right) \\
& +\operatorname{Exp}\left(-\lambda_{3} T\right) /\left(\lambda_{1}-\lambda_{3}\right) /\left(\lambda_{2}-\lambda_{3}\right) /\left(\lambda_{4}-\lambda_{3}\right) \\
& \left.+\operatorname{Exp}\left(-\lambda_{4} T\right) /\left(\lambda_{1}-\lambda_{4}\right) /\left(\lambda_{2}-\lambda_{4}\right) /\left(\lambda_{3}-\lambda_{4}\right)\right\}
\end{aligned}
$$


where,

$A_{1}^{0}, A_{2}^{0}, A_{3}^{0}, A_{4}^{0}=$ initial activity of nuclides $1,2,3$, and $4(T=0)$.

$A_{1}, A_{2}, A_{3}, A_{4}=$ activity of nuclides $1,2,3$, and 4 at time $T$.

$\lambda_{1}, \lambda_{2}, \lambda_{3}, \lambda_{4}=$ decay constants for nuclides $1,2,3$, and 4 . Note that the decay constant is the natural logarithm of 2 divided by the half-life of the nuclide. Note also that the decay constants in a decay chain are all different so the denominators will never be zero.

The accumulated dose at time $T$ is the time integral of the activity equations. These integrations are simply the integral of each exponential. In other words, each exponential in the decay equations, $\operatorname{Exp}(-\lambda T)$ is replaced with its integra,$[1-\operatorname{Exp}(-\lambda T)] / \lambda$.

Table A-3 shows the half-lives of nuclides used in the spreadsheet. The halflives are from Kocher (1981). Table A-3 also shows the internal dose-factors for each nuclide from EPA (1988 and 1993). Information on the imp Ticit daughters for nuclides with the "D" are listed at the end of Table A-3. The solubitity classes of these nuclides are also shown for reference. The dose factors for the implicit daughters are multiplied by the branching ratios listed in Table A-2 before being added to the parent dose factor.

In Table A-3 the "Lung" column refers to the rate of clearance from the pulmonary region of the lung according to the ICRP lung model. There are only three possibilities, "D" means days, "W" means weeks, and "Y" means years. Carbon ( $C-14)$ may be present as carbon dioxide, which is a gas. The ICRP uses a special modeT for carbon isotopes in this chemical form. These lung model choices are the worst-case assumptions, except for strontium, which uses class $D$ rather than class $Y$.

The "fl" column refers to the fraction of a stable element reaching body fluids following ingestion. These were also determined in ICRP 30 . A few elements (eg. cobalt an plutonium) have more than one value for $f l$, so the value which produces the largest internal dose was selected.

\section{DOSE AND SOIL LIMIT CALCULATION METHODS}

Over the course of a year, the worker is assumed to inhale the radioactivity in 12 grams of soil, and to ingest the radioactivity in 25 grams of soil. Starting with a soij concentration of $1 \mathrm{pCi} / \mathrm{g}$ for each nuclide, the worker inhales $12 \mathrm{pCi}$ and ingests $25 \mathrm{pC} i$ of each nuclide. The internal dose. commitment is shown in the equation below.

$$
\text { Dose }=[(12 \mathrm{pCi})(\mathrm{DF} \text { inhale })+(25 \mathrm{pCi}\rangle(\mathrm{DF} \text { ingest })] \text { (Decay Factor) }
$$

The inhalation and ingestion dose factors are shown on Table A-3. The decay factor is computed from the decay equations (integral form) where the initial activity of the parent is 1 and the initial activity of any progeny nuclides is zero. The decay and ingrowth period is 365.25 days. The computed annua 7 dose factors for a unit soil concentration are shown in the first column of numbers in Table 1.

The soil concentration 7 imits were computed by dividing the annual dose 7 imit by the dose from a unit soil concentration. Results were shown in the last three columns of Table 1 . 
Table A-1. Decay Chains Computed in the Spreadsheet

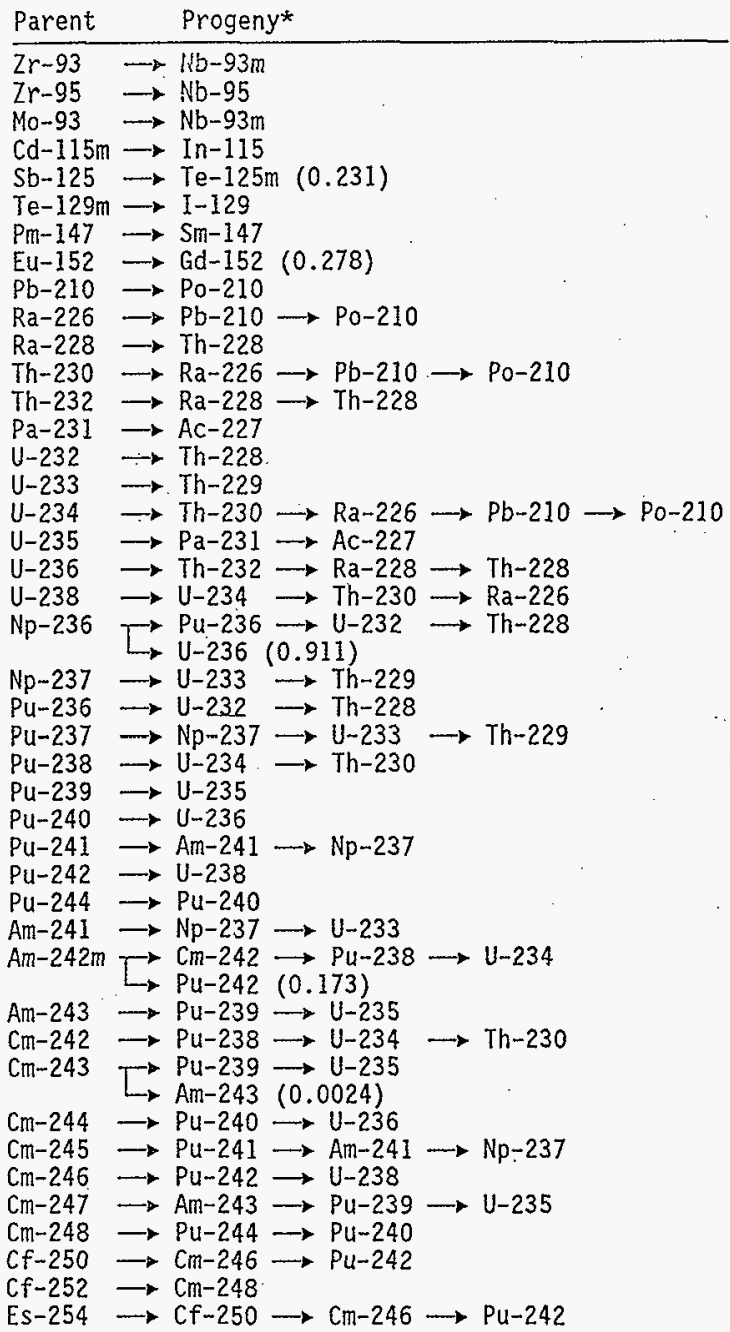

*The initial concentrations of these progeny are zero. During the exposure scenario (one year), their concentration increases. 
Table A-2. Composition of the " $+D$ " Nuclides

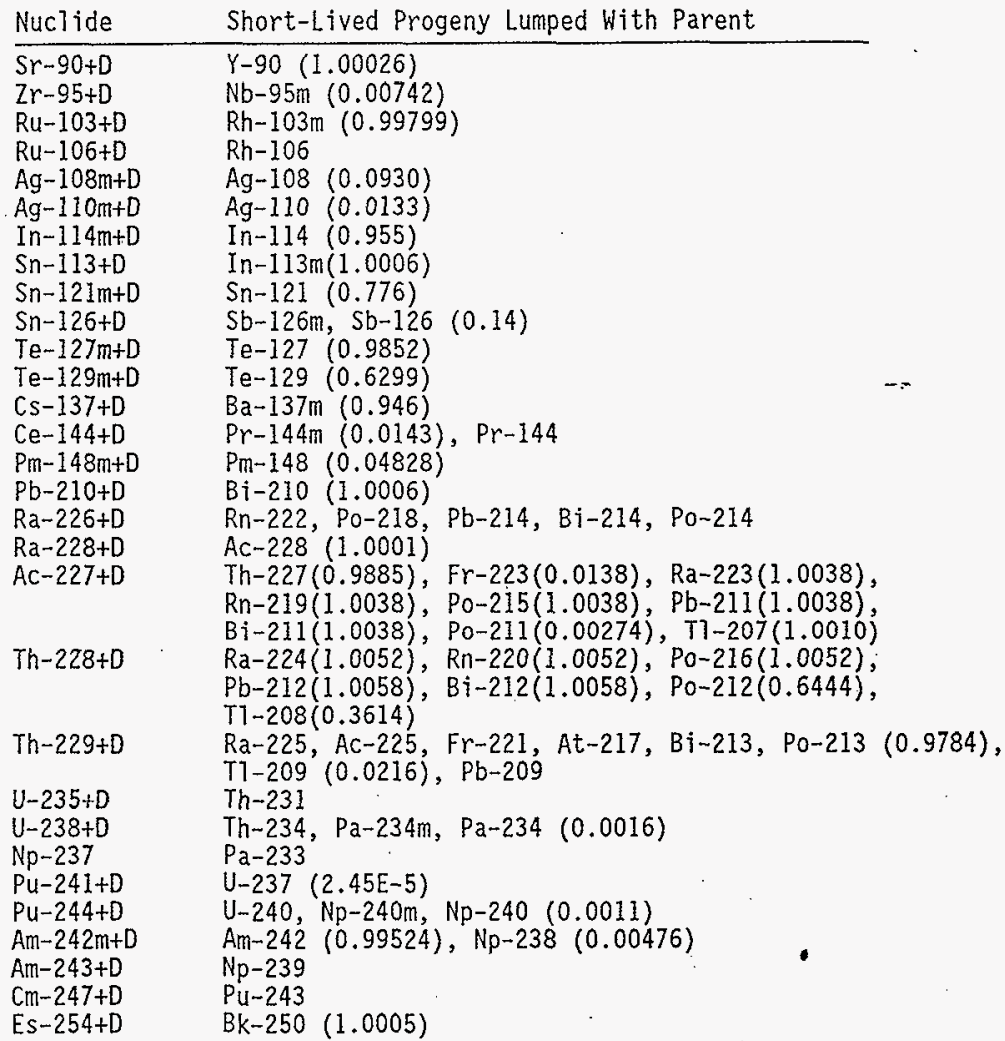

Any branching ratios different from 1.0 are shown in parentheses. The decay chain corrections are included.

These implicit daughters are assumed to be in equilibrium with the parent nuclide at all times.

Decay chains computed by the spreadsheet are shown in Table A-1. 
Table A-3. Solubility Assumptions, Half Lives and Dose Factors

\begin{tabular}{|c|c|c|c|c|c|c|}
\hline & & & & mrem $/ p$ & Intake & per pCi $/ 9$ \\
\hline Nuclide & Lung & f1 & Half-life & Ingest & Inhale & Externa \\
\hline $\mathrm{H}-3$ & & 1 & $12.28 Y$ & $6.40 \mathrm{E}-08$ & $9.60 \mathrm{E}-08$ & $0.00 E+00$ \\
\hline & $Y$ & 0.005 & $\quad 53.44 \mathrm{D}$ & 1.28E-07 & $3.21 \mathrm{E}-07$ & $3.28 \mathrm{E}-05$ \\
\hline & $W$ & 0.005 & $1.600 \mathrm{E}+06 \mathrm{Y}$ & $4.66 \mathrm{E}-06$ & $3.55 \mathrm{E}-04$ & $1.23 E-07$ \\
\hline & $\mathrm{CO}_{2}$ & 1 & $5,730 Y$ & $2.09 \mathrm{E}-06$ & $2.09 \mathrm{E}-06$ & \\
\hline $\mathrm{Na}-2$ & $0^{2}$ & 1 & $2.602 \mathrm{Y}$ & $1.15 \mathrm{E}-05$ & $7.66 \mathrm{E}-06$ & \\
\hline $\mathrm{S}$ & $W$ & 0.1 & $87.44 \mathrm{D}$ & $7.33 \mathrm{E}-07$ & $2.48 \mathrm{E}$ & $1.70 E-09$ \\
\hline $\bar{c}$ & W & 1 & $301,000 Y$ & $3.03 \mathrm{E}-06$ & $2.19 \mathrm{E}$ & $2.73 E-07$ \\
\hline & D & 1 & 1.277Et & $1.86 \mathrm{E}-05$ & 1.24 & $1.19 E-04$ \\
\hline & W & 0.3 & 103,0 & 1.27 & 1.3 & $0.00 E+00$ \\
\hline & $\ddot{W}$ & 0.3 & .70 & 3.16 & 6.6 & \\
\hline & $\ddot{Y}$ & 0.0001 & 80 & 6.40 & 2.9 & $5 E-03$ \\
\hline & W & 0.01 & $330 \mathrm{D}$ & 6.14 & 3.4 & $\overline{\mathrm{O}} \mathrm{E}+00$ \\
\hline & $\ddot{W}$ & 0.1 & $312.7 \mathrm{D}$ & 2.77 & 6.7 & \\
\hline & D & 0. & $2.7 Y$ & 6.07 & & 100 \\
\hline & D & 0. & $44.63 \mathrm{D}$ & 6.70 & 1.48 & $=-04$ \\
\hline & Y & 0.3 & $270.9 \mathrm{D}$ & 1.18 & 9.07 & 5.7 \\
\hline & $Y$ & 0.3 & $70.8 \mathrm{D}$ & 3.58 & 1.09 & \\
\hline & $Y$ & 0.3 & $5.271 \mathrm{Y}$ & 2.69 & 2.19 & $1.85 E-03$ \\
\hline & D & 0.05 & $75,000 Y$ & -07 & 1.33 & $0.00 \mathrm{E}+00$ \\
\hline & D & 0.05 & $100.1 Y$ & 5.77 & 3.10 & $0.00 E+00$ \\
\hline & $Y$ & 0.5 & 244.40 & 1.44 & 2.04 & $4.22 \mathrm{E}-04$ \\
\hline & $W$ & 0.8 & $119.78 \mathrm{D}$ & 9.62 & 8.47 & 2.2 \\
\hline & $W$ & 0.8 & $65,000 Y$ & 8.70 & 9.84 & 2.12 \\
\hline & D & & $4.730 E^{\circ}+10 Y$ & $4.92 E-06$ & 3.2 & 1.61 \\
\hline & D & 0.3 & $50.55 \mathrm{D}$ & $9.25 E$ & 6.51 & 1.04 \\
\hline & D & 0.3 & $28.6 Y$ & 1.53 & 2.48 & 2.8 \\
\hline & $Y$ & 0.0001 & $106.6 \mathrm{D}$ & 5.99 & 2.81 & 2.0 \\
\hline & $\dot{y}$ & 0.0 & $58.51 \mathrm{D}$ & 9.51 & & -06 \\
\hline & $\dot{D}$ & & $1.530 E+06 Y$ & $1.66 \mathrm{E}$ & 3.21 & -00 \\
\hline & D & & 64.020 & 3.798 & 2.37 & 5.1 \\
\hline & $Y$ & & $.6 \gamma$ & 5.22 & 2.9 & 1.1 \\
\hline & $Y$ & & 20,3 & 7.1 & 4.14 & 1.1 \\
\hline & Y & & D & 2.57 & 5.8 & 5.3 \\
\hline & Y & 0. & 8 & 1.3 & 2.8 & 6.7 \\
\hline & $W$ & 0.8 & 213 & 1.46 & 8.3 & 1.4 \\
\hline & $Y$ & 0.05 & 50 & 3.0 & 8.9 & 3.13 \\
\hline & $Y$ & 0 & 20 & 2.80 & 4.7 & 1.47 \\
\hline & $Y$ & 0.005 & $6.500 E t$ & 1.50 & 1.2 & 0.08 \\
\hline$n+D$ & $Y$ & & $7 \mathrm{Y}$ & 7.6 & 2.8 & 1.1 \\
\hline & $y$ & 0 & $249.85 \mathrm{D}$ & 1.0 & 8. & 1.96 \\
\hline & D & & $464 \mathrm{D}$ & 1.3 & 1.1 & 1.68 \\
\hline & D & 0 . & $7 \mathrm{Y}$ & 1.6 & 1.5 & 7.4 \\
\hline & D & 0 . & D & 1.62 & & \\
\hline$m+D$ & D & & 49 & 1.7 & & 5.7 \\
\hline & 0 & & 4. $600 E_{t}$ & 1.5 & & -08 \\
\hline & $W$ & & 10 & & & \\
\hline & $\because$ & & & & & -07 \\
\hline
\end{tabular}


Table A-3. Solubility Assumptions, Half Lives and Dose Factors, Continued

\begin{tabular}{|c|c|c|c|c|c|c|}
\hline \multirow[b]{2}{*}{ Nuclide } & \multirow[b]{2}{*}{ lung } & \multirow[b]{2}{*}{$f I$} & \multirow[b]{2}{*}{ Half-life } & \multicolumn{2}{|c|}{ mrem/oci Intake } & \multirow{2}{*}{$\begin{array}{l}\text { per pci/g } \\
\text { External }\end{array}$} \\
\hline & & & & Ingest & Inhale & \\
\hline$S n-121 m+D$ & $W$ & 0.02 & $55 Y$ & $2.25 E-06$ & $1.19 E-05$ & $2.41 E-07$ \\
\hline$S n-123$ & $W$ & 0.02 & $129.2 \mathrm{D}$ & $8.40 E-06$ & $3.25 \mathrm{E}-05$ & $5.78 \mathrm{E}-06$ \\
\hline$S n-126+D$ & $W$ & 0.02 & $100,000 Y$ & $2.11 E-05$ & $1.01 \mathrm{E}-04$ & $1.35 \mathrm{E}-03$ \\
\hline$S b-124$ & $w$ & $0 . \overline{1}$ & 60.20 & $1.01 \mathrm{E}-05$ & $2.52 E-05$ & $1.33 \mathrm{E}-03$ \\
\hline$S b-$ & $W$ & $0 . \overline{1}$ & $2.77 \mathrm{Y}$ & $2.81 E-06$ & $1.22 E-05$ & $2.79 E-04$ \\
\hline $\mathrm{Te}-123 \mathrm{~m}$ & $W$ & 0.2 & $119.7 \mathrm{D}$ & $5.66 \mathrm{E}-06$ & $1.06 \mathrm{E}-05$ & $7.16 \mathrm{E}-05$ \\
\hline$T e-125 m$ & W & 0.2 & 580 & $3.67 \mathrm{E}-06$ & $7.29 E-06$ & $1.73 \mathrm{E}-06$ \\
\hline $\mathrm{Te}-127 \mathrm{~m}+\mathrm{D}$ & $W$ & 0.2 & 1090 & $8.93 \mathrm{E}-06$ & $2.18 \mathrm{E}-05$ & $3.84 \mathrm{E}-06$ \\
\hline$T e-129 m+D$ & $W$ & 0.2 & $33.6 \mathrm{D}$ & $1.08 \mathrm{E}-05$ & $2.40 \mathrm{E}-05$ & $4.49 E-05$ \\
\hline$I-125$ & D & 1 & 60.140 & $3.85 \mathrm{E}-05$ & $2.42 \mathrm{E}-05$ & $1.89 \mathrm{E}-06$ \\
\hline $1-12$ & D. & 1 & $1.570 E+07 \mathrm{Y}$ & $2.76 \mathrm{E}-04$ & $1.74 \mathrm{E}-04$ & 1.48E-06 \\
\hline$C s-134$ & D & 1 & $2.062 \mathrm{Y}$ & $7.33 E-05$ & $4.63 E-05$ & $1.08 \mathrm{E}-03$ \\
\hline Cs -135 & D & 1 & $2.300 E+06 Y$ & $7.07 \mathrm{E}-06$ & $4.55 \mathrm{E}-06$ & 4.37E-09 \\
\hline$C s-137+D$ & D & 1 & $30.17 \mathrm{Y}$ & $5.00 E-05$ & $3.19 \mathrm{E}-05$ & $3.89 \mathrm{E}-04$ \\
\hline $\mathrm{Ba}-133$ & D & 0.1 & $10.5 Y$ & $3.40 \mathrm{E}-06$ & $7.81 \mathrm{E}-06$ & $2.26 E-04$ \\
\hline $\mathrm{Ce}-144+\mathrm{D}$ & $\bar{Y}$ & 0.0003 & 284.30 & $2.11 \mathrm{E}-05$ & $3.74 \mathrm{E}-04$ & $3.70 \mathrm{E}-05$ \\
\hline $\mathrm{Pm}-147$ & Y & 0.0003 & $2.6234 \mathrm{Y}$ & $1.05 E-06$ & $3.92 \mathrm{E}-05$ & 5.71E-09 \\
\hline$P m-148 m+D$ & $Y$ & 0.0003 & $41.3 \mathrm{D}$ & $8.18 \mathrm{E}-06$ & $2.31 \mathrm{E}-05$ & $1.39 \mathrm{E}-03$ \\
\hline $5 m-147$ & $W$ & 0.0003 & $1.069 E+11 Y$ & $1.85 E-04$ & $7.47 \mathrm{E}-02$ & $0.00 E+00$ \\
\hline$S m-151$ & W & 0.0003 & $90 \mathrm{Y}$ & $3.89 \mathrm{E}-07$ & $3.00 \mathrm{E}-05$ & $1.12 \mathrm{E}-10$ \\
\hline Eu-I50 & W & 0.001 & $35.8 Y$ & $6.36 E-06$ & $2.68 \mathrm{E}-04$ & $9.95 \mathrm{E}-04$ \\
\hline$E u-152$ & $W$ & 0.001 & $13.6 \mathrm{Y}$ & $6.48 \mathrm{E}-06$ & $2.21 E-04$ & $7.99 \mathrm{E}-04$ \\
\hline Eu-154 & W & 0.001 & $8.8 Y$ & $9.55 \bar{E}-06$ & $2.86 \mathrm{E}-04$ & $8.76 \mathrm{E}-04$ \\
\hline$E u-155$ & W & 0.001 & $4.96 \mathrm{Y}$ & $1.53 \mathrm{E}-06$ & $4.14 \mathrm{E}-05$ & $2.08 \mathrm{E}-05$ \\
\hline C $15 ?$ & $\ddot{D}$ & 0.0003 & $1.100 E+14 \dot{Y}$ & $1.61 \mathrm{E}-04$ & $2.44 \mathrm{E}-01$ & $0.00 E+00$ \\
\hline $6 d-153$ & D & 0.0003 & 241.60 & $1.17 \mathrm{E}-06$ & $2.38 E-05$ & $2.79 \mathrm{E}-05$ \\
\hline $\mathrm{Tb}-158$ & W & 0.0003 & $150 \mathrm{Y}$ & $4.40 \mathrm{E}-06$ & -04 & $5.39 \mathrm{E}-04$ \\
\hline$T b-160$ & W & 0.0003 & 72.30 & $6.73 E-06$ & $2.50 E-05$ & $7.89 \mathrm{E}-04$ \\
\hline $\mathrm{Ho}-166 \mathrm{~m}$ & $W$ & 0.0003 & $1200 Y$ & 8.07 & $7.73 E-04$ & 1.17E-03 \\
\hline$T m-170$ & W & 0.0003 & 128.60 & $5.29 \mathrm{E}-06$ & $2.63 \mathrm{E}-05$ & $1.71 \mathrm{E}-06$ \\
\hline $\mathrm{Ta}-182$ & $Y$ & 0.001 & $114.74 \mathrm{D}$ & $6.51 \mathrm{E}-06$ & & $9.06 \mathrm{E}-04$ \\
\hline$W-181$ & D & 0.01 & $120.95 \mathrm{D}$ & $3.45 \mathrm{E}-07$ & $-07^{\circ}$ & $8.72 E-06$ \\
\hline$W-185$ & D & 0.01 & 75.10 & $1.99 \mathrm{E}-06$ & $7.51 \mathrm{E}-07$ & $4.94 \mathrm{E}-08$ \\
\hline Re-187 & $W$ & 0.8 & $4.700 E+10 Y$ & $9.51 \mathrm{E}-09$ & $5.44 \mathrm{E}-08$ & $0.00 E+00$ \\
\hline $0 s-185$ & D & 0.01 & $93.6 \mathrm{D}$ & $2.26 \mathrm{E}-06$ & $1.04 \mathrm{E}-05$ & $4.75 \mathrm{E}-04$ \\
\hline 192 & Y & 0.01 & $74.02 \mathrm{D}$ & $5.74 \mathrm{E}-06$ & $2.82 E-05$ & $5.26 E-04$ \\
\hline$P t-193$ & 0 & 0.01 & $50 \mathrm{Y}$ & $1.19 \mathrm{E}-07$ & $2.27 \bar{E}-07$ & $6.46 \bar{E}-10$ \\
\hline$A u-195$ & $Y$ & 0.1 & $183 \mathrm{D}$ & $1.06 \mathrm{E}-06$ & 1. $30 \mathrm{E}-05$ & $2.37 \mathrm{E}-05$ \\
\hline $\mathrm{Hg}-203$ & D & 1 & 46.60 & 1.14E-05 & 7.33E-06 & $1.44 \mathrm{E}-04$ \\
\hline$T T-204$ & D & 1 & $3.779 \mathrm{Y}$ & & & $4.63 \mathrm{E}-07$ \\
\hline$P b-210+D$ & D & 0.2 & $22.26 \mathrm{Y}$ & $5.37 \mathrm{E}-03$ & $1.38 \mathrm{E}-02$ & $6.91 \mathrm{E}-07$ \\
\hline $8 i-207$ & $W$ & 0.05 & $33.4 \mathrm{Y}$ & & & $1.07 \mathrm{E}-03$ \\
\hline Po-209 & D & 0.1 & $102 \mathrm{Y}$ & $2.38 \mathrm{E}-03$ & $1.18 \mathrm{E}-02$ & $2.04 \mathrm{E}-02$ \\
\hline & D & 0.1 & $138.378 \mathrm{D}$ & $1.90 \mathrm{E}-03$ & $9.40 E-03$ & $5.97 \mathrm{E}-09$ \\
\hline$R a-226+D$ & $W$ & 0.2 & $1600 \mathrm{Y}$ & & & $1.28 \mathrm{E}-03$ \\
\hline$R a-228+D$ & W & 0.2 & $5.75 Y$ & $1.44 \mathrm{E}-03$ & $5.08 \mathrm{E}-03$ & $6.82 \mathrm{E}-04$ \\
\hline$A C-227+D$ & D & 0.001 & $21.773 \mathrm{Y}$ & $1.48 \mathrm{E}-02$ & $6.72 E+00$ & $2.30 \mathrm{E}-04$ \\
\hline$T h-228+D$ & Y & 0.0002 & $1.9132 Y$ & $8.11 E-04$ & $3.45 \mathrm{E}-01$ & $1.17 \mathrm{E}-03$ \\
\hline
\end{tabular}


Table A-3. Solubility Assumptions, Half Lives and Dose Factors; Continued

\begin{tabular}{|c|c|c|c|c|c|c|}
\hline \multirow[b]{2}{*}{ Nuclide } & \multirow[b]{2}{*}{ Lung } & \multirow[b]{2}{*}{$\mathrm{fl}$} & \multirow[b]{2}{*}{ Half-life } & \multicolumn{2}{|c|}{ mrem/oci Intake } & \multirow{2}{*}{$\begin{array}{l}\text { mrem } / \text { hour } \\
\text { per pCi } / 9 \\
\text { External }\end{array}$} \\
\hline & & & & Ingest & Inhale & \\
\hline 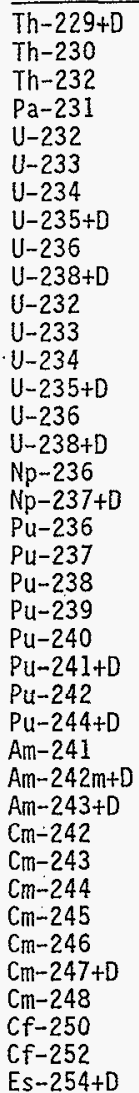 & $\begin{array}{l}W \\
W \\
W \\
W \\
W \\
W \\
W \\
W \\
W \\
W \\
Y \\
Y \\
Y \\
Y \\
Y \\
Y \\
W \\
W \\
W \\
Y \\
W \\
W \\
W \\
W \\
W \\
W \\
W \\
W \\
W \\
W \\
W \\
W \\
W \\
W \\
W \\
W \\
W \\
Y\end{array}$ & $\begin{array}{l}0.0002 \\
0.0002 \\
0.0002 \\
0.001 \\
0.05 \\
0.05 \\
0.05 \\
0.05 \\
0.05 \\
0.05 \\
0.05 \\
0.05 \\
0.05 \\
0.05 \\
0.05 \\
0.05 \\
0.001 \\
0.001 \\
0.001 \\
0.001 \\
0.001 \\
0.001 \\
0.001 \\
0.001 \\
0.001 \\
0.001 \\
0.001 \\
0.001 \\
0.001 \\
0.001 \\
0.001 \\
0.001 \\
0.001 \\
0.001 \\
0.001 \\
0.001 \\
0.001 \\
0.001\end{array}$ & 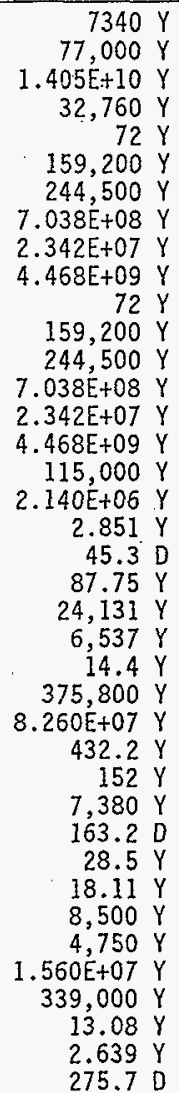 & $\begin{array}{l}2.73 \mathrm{E} \\
1.06 \mathrm{E} \\
1.31 \mathrm{E} \\
2.89 \mathrm{E} \\
2.83 \mathrm{E} \\
2.67 \mathrm{E} \\
2.69 \mathrm{E} \\
2.68 \mathrm{E} \\
1.31 \mathrm{E} \\
2.89 \mathrm{E} \\
2.83 \mathrm{E} \\
2.67 \mathrm{E}\end{array}$ & $\begin{array}{l}2.17 \mathrm{E}+00 \\
3.26 \mathrm{E}-01 \\
1.64 \mathrm{E}+00 \\
1.28 \mathrm{E}+00 \\
1.49 \mathrm{E}-02 \\
7.99 \mathrm{E}-03 \\
7.88 \mathrm{E}-03 \\
7.29 \mathrm{E}-03 \\
7.44 \mathrm{E}-03 \\
7.06 \mathrm{E}-03 \\
6.59 \mathrm{E}-01 \\
1.35 \mathrm{E}-01 \\
1.33 \mathrm{E}-01 \\
1.23 \mathrm{E}-01 \\
1.25 \mathrm{E}-01 \\
1.18 \mathrm{E}-01 \\
1.04 \mathrm{E}-01 \\
5.40 \mathrm{E}-01 \\
1.45 \mathrm{E}-01 \\
1.97 \mathrm{E}-06 \\
3.92 \mathrm{E}-01 \\
4.29 \mathrm{E}-01 \\
4.29 \mathrm{E}-01 \\
8.25 \mathrm{E}-03 \\
4.11 \mathrm{E}-01 \\
4.03 \mathrm{E}-01 \\
4.44 \mathrm{E}-01 \\
4.26 \mathrm{E}-01 \\
4.40 \mathrm{E}-01 \\
1.73 \mathrm{E}-02 \\
3.07 \mathrm{E}-01 \\
2.48 \mathrm{E}-01 . \\
4.55 \mathrm{E}-01 \\
4.51 \mathrm{E}-01 \\
4.14 \mathrm{E}-01 \\
1.65 \mathrm{E}+00 \\
2.62 \mathrm{E}-01 \\
1.57 \mathrm{E}-01 \\
4.11 \mathrm{E}-02\end{array}$ & $\begin{array}{l}1.82 \mathrm{E}-04 \\
1.38 \mathrm{E}-07 \\
5.95 \mathrm{E}-08 \\
2.17 \mathrm{E}-05 \\
1.03 \mathrm{E}-07 \\
1.59 \mathrm{E}-07 \\
4.58 \mathrm{E}-08 \\
8.64 \mathrm{E}-05 \\
2.45 \mathrm{E}-08 \\
1.51 \mathrm{E}-05 \\
1.03 \mathrm{E}-07 \\
1.59 \mathrm{E}-07 \\
4.58 \mathrm{E}-08 \\
8.64 \mathrm{E}-05 \\
2.45 \mathrm{E}-08 \\
1.51 \mathrm{E}-05 \\
5.26 \mathrm{E}-05 \\
1.25 \mathrm{E}-04 \\
2.60 \mathrm{E}-08 \\
1.85 \mathrm{E}-05 \\
1.73 \mathrm{E}-08 \\
3.37 \mathrm{E}-08 \\
1.67 \mathrm{E}-08 \\
2.16 \mathrm{E}-09 \\
1.46 \mathrm{E}-08 \\
2.31 \mathrm{E}-04 \\
4.99 \mathrm{E}-06 \\
7.72 \mathrm{E}-06 \\
1.02 \mathrm{E}-04 \\
1.95 \mathrm{E}-08 \\
6.65 \mathrm{E}-05 \\
1.44 \mathrm{E}-08 \\
3.88 \mathrm{E}-05 \\
1.33 \mathrm{E}-08 \\
2.12 \mathrm{E}-04 \\
1.00 \mathrm{E}-08 \\
1.35 \mathrm{E}-08 \\
2.01 \mathrm{E}-08 \\
6.35 \mathrm{E}-04\end{array}$ \\
\hline 10 & $\begin{array}{l}D \\
Y\end{array}$ & $\begin{array}{r}0.3 \\
0.0001\end{array}$ & & $1.08 \mathrm{E}-05$ & & \\
\hline $\begin{array}{l}-95 \\
-95 m-3 m\end{array}$ & $\begin{array}{l}D \\
Y\end{array}$ & 0.01 & & & & \\
\hline & $\begin{array}{l}Y \\
D\end{array}$ & & & $1.16 \mathrm{E}-08$ & $\begin{array}{l}8.95 \mathrm{E}-06 \\
5.11 \mathrm{E}-09\end{array}$ & $\begin{array}{l}3.13 \mathrm{E}-04 \\
2.77 \mathrm{E}-08\end{array}$ \\
\hline
\end{tabular}


Table A-3. Solubility Assumptions, Half Lives and Dose Factors, Continued

\begin{tabular}{|c|c|c|c|c|c|c|}
\hline \multirow[b]{2}{*}{ Nuclide } & \multirow[b]{2}{*}{ Lung } & \multirow[b]{2}{*}{ fl } & \multirow[b]{2}{*}{ Half-life } & \multicolumn{2}{|c|}{ mrem/pCi Intake } & \multirow{2}{*}{$\begin{array}{l}\text { mrem/hou } \\
\text { per pCi/ } \\
\text { External }\end{array}$} \\
\hline & & & & Ingest & Inhale & \\
\hline $\begin{array}{l}2 u-106+D \\
2 u-106 \\
2 h-106 \\
g-108 m+D\end{array}$ & $\begin{array}{l}Y \\
D\end{array}$ & $\begin{array}{l}0.05 \\
0.05\end{array}$ & $\begin{array}{r}368.2 \mathrm{D} \\
29.9 \mathrm{~S}\end{array}$ & $\begin{array}{l}2.74 E-05 \\
6.44 E-07\end{array}$ & $\begin{array}{l}4.77 E-04 \\
2.13 E-07\end{array}$ & $\begin{array}{l}0.00 \mathrm{E}+0 \\
1.47 \mathrm{E}-0\end{array}$ \\
\hline$m$ & $\begin{array}{l}Y \\
Y .\end{array}$ & $\begin{array}{l}0.05 \\
0.05\end{array}$ & $\begin{array}{r}127 Y \\
2.37 M\end{array}$ & 7.62E-06 & $2.83 E-04$ & $\begin{array}{l}1.10 \mathrm{E}-0 \\
1.30 \mathrm{E}-0\end{array}$ \\
\hline$m$ & $\begin{array}{l}y \\
y\end{array}$ & $\begin{array}{l}0.05 \\
0.05\end{array}$ & $\begin{array}{r}249.85 \mathrm{D} \\
24.6 \mathrm{~S}\end{array}$ & $1.08 \mathrm{E}-05$ & $8.03 E-05$ & $\begin{array}{l}1.96 \mathrm{E}-0 \\
2.56 \mathrm{E}-0\end{array}$ \\
\hline $\begin{array}{l}n-1 \\
n-1 \\
n-1\end{array}$ & $\begin{array}{l}D \\
D\end{array}$ & $\begin{array}{l}0.02 \\
0.02\end{array}$ & $\begin{array}{r}49.51 \mathrm{D} \\
71.9 \mathrm{~S}\end{array}$ & $\begin{array}{l}1.71 \mathrm{E}-05 \\
0.00 \mathrm{E}+00\end{array}$ & $\begin{array}{l}8.88 E-05 \\
0.00 E+00\end{array}$ & $\begin{array}{l}5.52 \mathrm{E}-0 \\
2.02 \mathrm{E}-0\end{array}$ \\
\hline$B+D$ & $\begin{array}{l}W \\
D\end{array}$ & $\begin{array}{l}0.02 \\
0.02\end{array}$ & $\begin{array}{r}115.1 \mathrm{D} \\
1.66 \mathrm{H}\end{array}$ & $\begin{array}{l}3.08 \mathrm{E}-06 \\
1.05 \mathrm{E}-07\end{array}$ & $\begin{array}{l}1.07 E-05 \\
4.11 E-08\end{array}$ & $\begin{array}{l}3.39 \mathrm{E}-0 \\
1.64 \mathrm{E}-0\end{array}$ \\
\hline $\begin{array}{l}n-1 \\
n-1 \\
n-1\end{array}$ & $\underset{W}{W}$ & $\begin{array}{l}0.02 \\
0.02\end{array}$ & $\begin{array}{l}55.5 \mathrm{Y} \\
27.1 \mathrm{H}\end{array}$ & $\begin{array}{l}1.55 \mathrm{E}-06 \\
9.03 \mathrm{E}-07\end{array}$ & & $\begin{array}{l}2.24 \mathrm{E}-0 \\
2.24 \mathrm{E}-0\end{array}$ \\
\hline $\begin{array}{l}-126 \\
-126 m \\
-126 \\
-127 m+D\end{array}$ & $\begin{array}{l}W \\
D \\
W\end{array}$ & $\begin{array}{r}0.02 \\
0.1 \\
0.1\end{array}$ & $\begin{array}{r}100,000 \mathrm{Y} \\
19 \mathrm{M} \\
12.4 \mathrm{D}\end{array}$ & $\begin{array}{l}1.95 \mathrm{E}-05 \\
9.40 \mathrm{E}-08 \\
1.07 \mathrm{E}-05\end{array}$ & & $\begin{array}{l}1.68 \mathrm{E}-05 \\
1.06 \mathrm{E}-03 \\
1.95 \mathrm{E}-03\end{array}$ \\
\hline $\begin{array}{l}\text { Te-127m } \\
\text { Te-127 }\end{array}$ & $\begin{array}{l}W \\
W\end{array}$ & $\begin{array}{l}0.2 \\
0.2\end{array}$ & & $\begin{array}{l}8.25 \mathrm{E}-06 \\
6.92 \mathrm{E}-07\end{array}$ & & \\
\hline $\begin{array}{l}-129 m \\
-129 \\
-137+D\end{array}$ & $\begin{array}{l}W \\
D\end{array}$ & $\begin{array}{l}0.2 \\
0.2\end{array}$ & & $\begin{array}{l}1.07 E-05 \\
2.02 E-07\end{array}$ & & $\begin{array}{l}2.11 \mathrm{E}-0 \\
3.77 \mathrm{E}-0\end{array}$ \\
\hline $\begin{array}{l}-137 \\
-137 . \mathrm{m} \\
-144+\mathrm{D}\end{array}$ & $\begin{array}{l}\text { D } \\
D\end{array}$ & $\begin{array}{r}1 \\
0.1\end{array}$ & & $\begin{array}{l}5.00 \mathrm{E}-05 \\
0.00 \mathrm{E}+00\end{array}$ & $\begin{array}{l}3.19 \mathrm{E}-05 \\
0.00 \mathrm{E}+00 .\end{array}$ & $\begin{array}{l}8.57 \mathrm{E}-0 \\
4.11 \mathrm{E}-0\end{array}$ \\
\hline$m$ & $\begin{array}{l}Y \\
Y\end{array}$ & & & & & \\
\hline & $Y$ & & & -07 & & \\
\hline $\begin{array}{l}-148 m \\
-148\end{array}$ & $\begin{array}{l}Y \\
Y\end{array}$ & $\begin{array}{l}0.0003 \\
0.0003\end{array}$ & & $\begin{array}{l}7.66 \mathrm{E}-06 \\
1.09 \mathrm{E}-05\end{array}$ & & $\begin{array}{l}1.37 \mathrm{E}-03 \\
4.20 \mathrm{E}-04\end{array}$ \\
\hline $\begin{array}{l}210 \\
-210 \\
226+D\end{array}$ & W & $\begin{array}{r}0.2 \\
0.05\end{array}$ & & $\begin{array}{l}5.37 E-03 \\
6.40 E-06\end{array}$ & & $\begin{array}{l}2.79 E-07 \\
4.11 E-07\end{array}$ \\
\hline $\begin{array}{l}-226 \\
-222 \\
-218 \\
-214 \\
-214 \\
-214\end{array}$ & $\begin{array}{l}W \\
D \\
D \\
W\end{array}$ & $\begin{array}{r}0.2 \\
1 \\
0.1 \\
0.2 \\
0.05 \\
0.1\end{array}$ & $\begin{array}{r}1,600 \mathrm{Y} \\
3.8235 \mathrm{D} \\
3.05 \mathrm{M} \\
26.8 \mathrm{M} \\
19.9 \mathrm{M} \\
0.0001637 \mathrm{~S}\end{array}$ & $\begin{array}{l}1.32 E-03 \\
9.08 E-07 \\
0.00 E+00 \\
6.25 E-07 \\
2.83 E-07 \\
0.00 E+00\end{array}$ & $\begin{array}{l}8.58 E-03 \\
1.44 E-05 \\
0.00 E+00 \\
7.81 E-06 \\
6.59 E-06 \\
0.00 E+00\end{array}$ & $\begin{array}{l}3.62 E-06 \\
2.69 E-07 \\
6.44 E-09 \\
1.53 E-04 \\
1.12 E-03 \\
5.86 E-08\end{array}$ \\
\hline
\end{tabular}


Table A-3. Solubility Assumptions, Half Lives and Dose Factors, Continued

\begin{tabular}{|c|c|c|c|c|c|c|}
\hline \multirow[b]{2}{*}{ Nuclide } & \multirow[b]{2}{*}{ Lung } & \multirow[b]{2}{*}{$f 1$} & \multirow[b]{2}{*}{ Half-life } & \multicolumn{2}{|c|}{$\mathrm{mrem} / \mathrm{pCi}$ Intake } & \multirow{2}{*}{$\begin{array}{l}\text { mrem/hou } \\
\text { per pci/ } \\
\text { External }\end{array}$} \\
\hline & & & & Ingest & Inhale & \\
\hline $\begin{array}{l}R a-228+D \\
R a-228 \\
A c-228 \\
A c-227+D\end{array}$ & $\begin{array}{l}W \\
D\end{array}$ & $\begin{array}{r}0.2 \\
0.001\end{array}$ & $\begin{array}{l}5.75 \mathrm{Y} \\
6.13 \mathrm{H}\end{array}$ & $\begin{array}{l}1.44 E-03 \\
2.16 E-06\end{array}$ & $\begin{array}{l}4.77 E-03 \\
3.08 E-04\end{array}$ & $\begin{array}{l}0.00 E+00 \\
6.82 E-04\end{array}$ \\
\hline$A C-227$ & D & 0.001 & $21.773 Y$ & $1.41 \mathrm{E}-02$ & $6.70 \mathrm{E}+00$ & $5.65 E-08$ \\
\hline$T h-22$ & $Y$ & 0.0002 & $18.718 \mathrm{D}$ & $3.81 \mathrm{E}-05$ & $1.62 E-02$ & $5.95 \mathrm{E}-05$ \\
\hline & D & & $21.8 \mathrm{M}$ & $8.25 \mathrm{E}-06$ & $6.22 \mathrm{E}-06$ & $2.26 \mathrm{E}-05$ \\
\hline $\mathrm{Ra}-2$ & W & 0.2 & $11.434 \mathrm{D}$. & $6.59 \mathrm{E}-04$ & $7.84 E-03$ & $6.88 \mathrm{E}-0$ \\
\hline-2 & & & $3.95 \mathrm{~S}$ & $0.00 E+00$ & $0.00 E+00$ & $3.52 \mathrm{E}-0$ \\
\hline-2. & $W$ & 0.1 & $001778 \mathrm{~S}$ & $0 E+00$ & $0.00 E+00$ & $1.16 \mathrm{E}-07$ \\
\hline $\mathrm{Pb}-2$ & D & 0.2 & $36.1 \mathrm{M}$ & $5.25 E-07$ & $8.70 \mathrm{E}-06$ & $3.50 E-05$ \\
\hline$B i-2$ & W & 0.05 & $2.13 \mathrm{M}$ & $0.00 E+\infty 0$ & $0.00 E+00$ & $2.92 \mathrm{E}-05$ \\
\hline$T 1-2$ & D & 1 & $4.77 \mathrm{M}$ & $0.00 E+00$ & $0 E+00$ & $2.26 \mathrm{E}-06$ \\
\hline 1 & & \\
\hline Th-2? & $Y$ & 0.0002 & $1.9132 Y$ & $3.96 E \sim 04$ & $3.42 E-01$ & $9.06 \mathrm{E}-07$ \\
\hline 2 & W & 0.2 & 520 & & & $34 \mathrm{E}-06$ \\
\hline-2 & & & $55.61 \mathrm{~S}$ & $0.00 E+00$ & $0 E+00$ & $62 \mathrm{E}-07$ \\
\hline 6 & W & 0.1 & $6 \mathrm{~S}$ & & & 19E-08 \\
\hline-2 & D & 0.2 & $10.643 \mathrm{H}$ & $E-05$ & 1.6 & $8.03 \mathrm{E}-05$ \\
\hline 2 & D & 0.05 & $60.55 \mathrm{M}$ & & & \\
\hline-2 & $W$ & 0.1 & $.98 \mathrm{E}-07 \mathrm{~S}$ & 0.0 & & $0.00 \mathrm{E}$ \\
\hline-2 & D & & $3.053 \mathrm{M}$ & $0.00 \mathrm{E}+00$ & $0.00 \bar{E}+00$ & $2.62 \mathrm{E}-03$ \\
\hline \multicolumn{7}{|c|}{7,340} \\
\hline$n=-2 c$ & W & $\begin{array}{r}0002 \\
0.2\end{array}$ & $\begin{array}{r}7,340 Y \\
14.8 \mathrm{D}\end{array}$ & & & $\begin{array}{l}3.8 \\
1.2\end{array}$ \\
\hline-28 & D & 0.001 & $\begin{array}{r}10.00 \\
10 \mathrm{D}\end{array}$ & 34 & & \\
\hline-22 & D & & 4.8 & 0.0 & & $1.75 \mathrm{E}-05$ \\
\hline-21 & D & 1 & 0.0323 & & & $2.02 \mathrm{E}-07$ \\
\hline & D & 0.05 & & & & \\
\hline & W & 0.1 & $4.2 E-06 S$ & & & 0.001 \\
\hline & 0 & 1 & & & & \\
\hline$x-2$ & D & 0.2 & $3.253 \mathrm{H}$ & $E-07$ & EE-08 & 8.821 \\
\hline \multicolumn{7}{|l|}{$\begin{array}{l}\mathrm{U}-235+\mathrm{D} \\
-235\end{array}$} \\
\hline J-235 & $W$ & $\begin{array}{r}0.05 \\
0.0002\end{array}$ & $\begin{array}{r}7.04 E+08 Y \\
25.52 H\end{array}$ & & & \\
\hline & & & & & & \\
\hline & W & 0.05 & $4.47 E+09 Y$ & & & \\
\hline Th- & W & & & & & \\
\hline $234 m$ & $W$ & & 1. & & & \\
\hline $\mathrm{Pa}-234$ & $w$ & & & $3.46 E-09$ & 1.17E-09 & $1.32 E-03$ \\
\hline$J-235$ & Y & & 7. & & & \\
\hline & $y$ & 0.0002 & $20.06 \pi$ & & & $4.16 \mathrm{E}-06$ \\
\hline & & & & & & \\
\hline & $Y$ & & & & & \\
\hline & Y & 0.0002 & 24.10 & & & \\
\hline & $\begin{array}{l}Y \\
y\end{array}$ & & $1.17 \mathrm{M}$ & & & 1.9 \\
\hline & & & & $2.16 E-06$ & & \\
\hline
\end{tabular}


Table A-3. Solubility Assumptions, Half Lives and Dose Factors, Continued

\begin{tabular}{|c|c|c|c|c|c|c|}
\hline & & & & $\mathrm{mrem} / \mathrm{po}$ & Intake & $\begin{array}{l}\text { mrem } / \text { hour } \\
\text { per } \mathrm{pCi} / \mathrm{s}\end{array}$ \\
\hline Nuclide & Lung & fl & Half-life & Ingest & Inhale & \\
\hline$N p-237+D$ & & & & & & \\
\hline$N p-237$ & W & 0.001 & $2.14 E+06 Y$ & $4.44 \mathrm{E}-03$ & $5.40 \mathrm{E}-01$ & $8.89 \mathrm{E}-06$ \\
\hline $\begin{array}{l}\mathrm{Pa}-233 \\
\mathrm{Pu}-241+\mathrm{D}\end{array}$ & Y & 0.001 & $27 \mathrm{D}$ & $3.63 \mathrm{E}-06$ & $9.55 \mathrm{E}-06$ & $1.16 E-04$ \\
\hline Pu-241 & W & 0.001 & $14.4 Y$ & $6.85 \mathrm{E}-05$ & $8.25 E-03$ & $6.73 \mathrm{E}-10$ \\
\hline$U-237$ & $\ddot{\gamma}$ & 0.05 & 6.75 .0 & $3.14 \mathrm{E}-06$ & $3.53 E-06$ & $6.05 \mathrm{E}-05$ \\
\hline$P U-244+D$ & & & & & & \\
\hline$P u-244$ & W & 0.001 & $8.26 \mathrm{E}+07 \mathrm{Y}$ & $3.32 E-03$ & $4.03 E-01$ & 8.61E-09 \\
\hline$U-240$ & Y & 0.05 & $14.1 \mathrm{H}$ & $4.29 E-06$ & $2.27 E-06$ & $1.62 E-07$ \\
\hline $\mathrm{Np}-240 \mathrm{~m}$ & $W$ & 0.001 & $7.4 \mathrm{M}$ & $0.00 E+00$ & $0.00 E+00$ & $2.30 \mathrm{E}-04$ \\
\hline $\begin{array}{l}N p-240 \\
A m-242 m+D\end{array}$ & W & 0.001 & - $\quad 65 \mathrm{M}$ & $2.37 E-07$ & $8.14 \mathrm{E}-08$ & $8.82 E-04$ \\
\hline$A m-242 m$ & W & 0.001 & $152 Y$ & $3.52 \mathrm{E}-03$ & $4.26 \mathrm{E}-01$ & $1.93 \mathrm{E}-07$ \\
\hline$A m-242$ & W & 0.001 & $16.02 \mathrm{H}$ & $1.41 \mathrm{E}-06$ & $5.85 E-05$ & $5.69 \mathrm{E}-06$ \\
\hline $\mathrm{Np}-238$ & W & 0.001 & $2.117 \mathrm{D}$ & $4.00 E-06$ & $3.70 E-05$ & $3.92 E-04$ \\
\hline$A m-243+D$ & & & & & & \\
\hline$A m-243$ & W & 0.001 & $7,380 \mathrm{Y}$ & $3.62 \mathrm{E}-03$ & $4.40 E-01$ & $1.62 E-05$ \\
\hline $\mathrm{Np}-239$ & $W$ & 0.001 & 2.3550 & $3.26 \mathrm{E}-06$ & $2.51 \mathrm{E}-06$ & $8.59 E-05$ \\
\hline$C m-247+D$ & & & & & & \\
\hline $\mathrm{Cm}-247$ & $W$ & 0.001 & $1.56 \mathrm{E}+07 \mathrm{Y}$ & $3.42 E-03$ & $4.14 \mathrm{E}-01$ & $2.03 E-04$ \\
\hline $\mathrm{Pu}-243$ & $Y$ & 0.001 & . & $3.33 \mathrm{E}-07$ & $1.64 \mathrm{E}-07$ & $9.08 E-06$ \\
\hline Es-254 & $W$ & 0 & & & & \\
\hline$B k-250$ & W & 0.001 & $3.222 \mathrm{H}$ & $5.81 \mathrm{E}-07$ & $7.55 \mathrm{E}-06$ & $6.33 E-04$ \\
\hline
\end{tabular}

Note: Half-life data is from Kocher (1981). Internal dose factors are from EPA (1988). External dose rate factors are from EPA (1993) for an infinite depth of soil with a density of $1.6 \mathrm{~g} / \mathrm{cc}$.

The composition of the "Pu $6 \%$ " is shown in Table A-4. It is a typical lowenrichment mixture of plutonium isotopes. The relative activity is as weighting factors when combining dose factors for individual plutonium isotopes to arrive at the unit dose factor for the mixture. Since the Pu-241 is a beta-emitting nuclide, its activity is not included in the total shown on the table.

Table A-4. Assumed Composition of Plutonium 6\% Mixture

\begin{tabular}{lrcrc} 
& $\begin{array}{c}\text { Fresh Mixture } \\
\text { Weight } \\
\text { Relative } \\
\text { Activity }\end{array}$ & $\begin{array}{c}\text { Decayed } \\
\text { Weight } \\
\text { Percent }\end{array}$ & $\begin{array}{c}\text { Relative } \\
\text { Retivity }\end{array}$ \\
\hline Pu-238 & 0.0298 & 0.0623 & 0.0275 & 0.0531 \\
Pu-239 & 93.1854 & 0.7051 & 93.1592 & 0.6498 \\
Pu-240 & 6.0328 & 0.1670 & 6.0265 & 0.1538 \\
Pu-241 & 0.5724 & 7.2000 & 0.3537 & 4.1010 \\
Pu-242 & 0.0230 & $1.1 E-5$ & 0.0230 & $1.0 \mathrm{E}-5$ \\
Am-241 & 0.1566 & 0.0656 & 0.3709 & 0.1433 \\
\hline Total & 100.0000 & 1.0000 & 99.9608 & 1.0000
\end{tabular}




\section{Appendix B: Method for Estimating Field Instrument Readings}


Three field instruments are considered in this report, the window-closed CP, the GM with a pancake probe $\left(15 \mathrm{~cm}^{2}\right.$ face area), and the Portable Alpha Monitor (PAM) $\left(50 \mathrm{~cm}^{2}\right.$ face area). To estimate the detector readings for particular soil contaminations, several assumptions were made about these detectors. The $C P$ is assumed to respond only to photon radiation. The GM and PAM are assumed to ignore photons and respond only to charged particles. The GM is assumed to respond to any charged particle able to enter the active volume of the detector. The PAM is assumed to respond only to alpha particles with enough energy to enter the detector. All three detectors are assumed to respond equally to all radiation energies above $10 \mathrm{keV}$. In other words, the detectors are assumed to have a flat energy response.

\section{PHOTON READINGS HITH A CP}

The field reading for the $C P$ are based on the computed external dose rate one meter above an infinite slab of contaminated soil which is quite thick. Contaminated areas larger than $100 \mathrm{~m}^{2}$ produce nearly the same dose rates as the infinite layer. Examples computed with ISO-PC Version 1.98 are provided below in Table B-1.

\section{Table B-1. Dose Rates 1 meter Above Various Circular Areas}

Area Radius Dose Rates (rem/h per $\mu \mathrm{Ci} / \mathrm{cc}$ ) for Various Photon Energies

\begin{tabular}{rccccccccc}
$\mathrm{m}^{2}$ & $\mathrm{~cm}$ & $.02 \mathrm{MeV}$ & $.05 \mathrm{MeV}$ & $0.1 \mathrm{MeV}$ & $0.2 \mathrm{MeV}$ & $0.5 \mathrm{MeV}$ & $1 \mathrm{MeV}$ & $2 \mathrm{MeV}$ \\
\hline \hline 0.100 & 17.84 & $3.3 \mathrm{E}-06$ & $9.9 \mathrm{E}-05$ & $3.5 \mathrm{E}-04$ & $1.0 \mathrm{E}-03$ & $3.2 \mathrm{E}-03$ & $6.4 \mathrm{E}-03$ & $1.3 \mathrm{E}-02$ \\
0.215 & 26.19 & $6.9 \mathrm{E}-06$ & $2.1 \mathrm{E}-04$ & $7.4 \mathrm{E}-04$ & $2.2 \mathrm{E}-03$ & $6.8 \mathrm{E}-03$ & $1.4 \mathrm{E}-02$ & $2.7 \mathrm{E}-02$ \\
0.454 & 38.44 & $1.4 \mathrm{E}-05$ & $4.3 \mathrm{E}-04$ & $1.5 \mathrm{E}-03$ & $4.5 \mathrm{E}-03$ & $1.4 \mathrm{E}-02$ & $2.8 \mathrm{E}-02$ & $5.6 \mathrm{E}-02$ \\
1.00 & 56.42 & $2.7 \mathrm{E}-05$ & $8.4 \mathrm{E}-04$ & $3.0 \mathrm{E}-03$ & $8.9 \mathrm{E}-03$ & $2.8 \mathrm{E}-02$ & $5.6 \mathrm{E}-02$ & $1.1 \mathrm{E}-01$ \\
2.15 & 82.81 & $4.8 \mathrm{E}-05$ & $1.5 \mathrm{E}-03$ & $5.5 \mathrm{E}-03$ & $1.6 \mathrm{E}-02$ & $5.2 \mathrm{E}-02$ & $1.0 \mathrm{E}-01$ & $2.1 \mathrm{E}-01$ \\
4.64 & 121.6 & $7.6 \mathrm{E}-05$ & $2.4 \mathrm{E}-03$ & $8.9 \mathrm{E}-03$ & $2.7 \mathrm{E}-02$ & $8.6 \mathrm{E}-02$ & $1.8 \mathrm{E}-01$ & $3.6 \mathrm{E}-01$ \\
10.0 & 178.4 & $1.1 \mathrm{E}-04$ & $3.4 \mathrm{E}-03$ & $1.3 \mathrm{E}-02$ & $3.9 \mathrm{E}-02$ & $1.3 \mathrm{E}-01$ & $2.6 \mathrm{E}-01$ & $5.3 \mathrm{E}-01$ \\
21.5 & 261.9 & $1.3 \mathrm{E}-04$ & $4.3 \mathrm{E}-03$ & $1.6 \mathrm{E}-02$ & $5.0 \mathrm{E}-02$ & $1.6 \mathrm{E}-01$ & $3.4 \mathrm{E}-01$ & $7.0 \mathrm{E}-01$ \\
46.4 & 384.4 & $1.5 \mathrm{E}-04$ & $5.0 \mathrm{E}-03$ & $1.9 \mathrm{E}-02$ & $5.9 \mathrm{E}-02$ & $1.9 \mathrm{E}-01$ & $4.0 \mathrm{E}-01$ & $8.3 \mathrm{E}-01$ \\
100 & 564.2 & $1.7 \mathrm{E}-04$ & $5.6 \mathrm{E}-03$ & $2.1 \mathrm{E}-02$ & $6.5 \mathrm{E}-02$ & $2.1 \mathrm{E}-01$ & $4.4 \mathrm{E}-01$ & $9.2 \mathrm{E}-01$ \\
215 & 828.1 & $1.7 \mathrm{E}-04$ & $5.9 \mathrm{E}-03$ & $2.2 \mathrm{E}-02$ & $6.9 \mathrm{E}-02$ & $2.3 \mathrm{E}-01$ & $4.7 \mathrm{E}-01$ & $9.9 \mathrm{E}-01$ \\
464 & 1,216 & $1.8 \mathrm{E}-04$ & $6.2 \mathrm{E}-03$ & $2.3 \mathrm{E}-02$ & $7.2 \mathrm{E}-02$ & $2.4 \mathrm{E}-01$ & $4.9 \mathrm{E}-01$ & $1.0 \mathrm{E}+00$ \\
1,000 & 1,784 & $1.8 \mathrm{E}-04$ & $6.3 \mathrm{E}-03$ & $2.4 \mathrm{E}-02$ & $7.4 \mathrm{E}-02$ & $2.4 \mathrm{E}-01$ & $5.1 \mathrm{E}-01$ & $1.1 \mathrm{E}+00$ \\
10,000 & 5,642 & $1.9 \mathrm{E}-04$ & $6.5 \mathrm{E}-03$ & $2.5 \mathrm{E}-02$ & $7.6 \mathrm{E}-02$ & $2.5 \mathrm{E}-01$ & $5.2 \mathrm{E}-01$ & $1.1 \mathrm{E}+00$ \\
\hline Infinite & $1.9 \mathrm{E}-04$ & $6.6 \mathrm{E}-03$ & $2.5 \mathrm{E}-02$ & $7.7 \mathrm{E}-02$ & $2.5 \mathrm{E}-01$ & $5.3 \mathrm{E}-01$ & $1.1 \mathrm{E}+00$ \\
\hline \hline
\end{tabular}

Note: The contamination thickness is 1.5 meters in all cases.

The external dose rates from EPA (1993) are preferred for estimating dose rates above contaminated soil because a more detailed calculation was carried out. However, the units are dose equivalent rather than exposure, which the $C P$ uses. For this reason, the ISO-PC program was used with both sets of units to develop ratios between the two sets of units for each nuclide. The dose results and $R /$ rem ratios are shown in Table $B-2$ below. These $R /$ rem ratios were multiplied by the external dose rate factors in Table A-3 and also by the soil contamination level to give the estimated exposure rate which is assumed to represent the $C P$ reading. The projected $C P$ readings were shown in Tables 2,3 , and 4 . 
Table B-2. R/rem Ratios and Detection Fractions

\begin{tabular}{|c|c|c|c|c|c|}
\hline Nuclide & $\begin{array}{l}\text { Results } \\
\text { mrem/h }\end{array}$ & er $\begin{array}{c}\mathrm{pCj} / \mathrm{g} \\
\mathrm{mR} / \mathrm{h}\end{array}$ & $\begin{array}{l}\text { R/rem } \\
\text { Ratio }\end{array}$ & $\begin{array}{l}\text { Detection, } \\
\text { GM P-11 }\end{array}$ & $\begin{array}{r}\mathrm{g} / \mathrm{cm}^{2} \\
\text { PAM }\end{array}$ \\
\hline 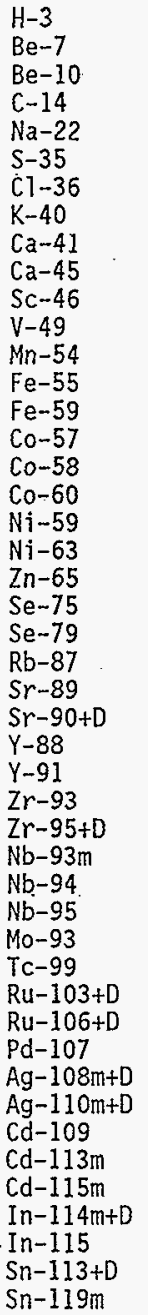 & $\begin{array}{l}5.70 \mathrm{E}-15 \\
3.65 \mathrm{E}-05 \\
1.75 \mathrm{E}-07 \\
2.75 \mathrm{E}-09 \\
1.64 \mathrm{E}-03 \\
3.33 \mathrm{E}-09 \\
3.15 \mathrm{E}-07 \\
1.26 \mathrm{E}-04 \\
0.00 \mathrm{E}+00 \\
1.54 \mathrm{E}-08 \\
1.50 \mathrm{E}-03 \\
0.00 \mathrm{E}+00 \\
6.37 \mathrm{E}-04 \\
0.00 \mathrm{E}+00 \\
9.30 \mathrm{E}-04 \\
6.63 \mathrm{E}-05 \\
6.61 \mathrm{E}-04 \\
1.87 \mathrm{E}-03 \\
0.00 \mathrm{E}+00 \\
3.28 \mathrm{E}-11 \\
4.49 \mathrm{E}-04 \\
2.44 \mathrm{E}-04 \\
1.82 \mathrm{E}-09 \\
1.71 \mathrm{E}-08 \\
2.11 \mathrm{E}-06 \\
5.78 \mathrm{E}-06 \\
1.97 \mathrm{E}-03 \\
4.77 \mathrm{E}-06 \\
2.15 \mathrm{E}-11 \\
5.43 \mathrm{E}-04 \\
9.40 \mathrm{E}-09 \\
1.11 \mathrm{E}-03 \\
5.47 \mathrm{E}-04 \\
5.27 \mathrm{E}-08 \\
2.16 \mathrm{E}-08 \\
3.39 \mathrm{E}-04 \\
1.55 \mathrm{E}-04 \\
4.36 \mathrm{E}-13 \\
1.14 \mathrm{E}-03 \\
2.00 \mathrm{E}-03 \\
2.27 \mathrm{E}-07 \\
1.65 \mathrm{E}-07 \\
1.81 \mathrm{E}-05 \\
8.83 \mathrm{E}-05 \\
1.02 \mathrm{E}-07 \\
1.73 \mathrm{E}-04 \\
2.40 \mathrm{E}-07\end{array}$ & $\begin{array}{l}1.59 \mathrm{E}-14 \\
5.62 \mathrm{E}-05 \\
3.24 \mathrm{E}-07 \\
5.74 \mathrm{E}-09 \\
2.33 \mathrm{E}-03 \\
6.91 \mathrm{E}-09 \\
5.73 \mathrm{E}-07 \\
1.69 \mathrm{E}-04 \\
0.00 \mathrm{E}+00 \\
3.04 \mathrm{E}-08 \\
2.10 \mathrm{E}-03 \\
0.00 \mathrm{E}+00 \\
9.10 \mathrm{E}-04 \\
0.00 \mathrm{E}+00 \\
1.27 \mathrm{E}-03 \\
1.18 \mathrm{E}-04 \\
9.70 \mathrm{E}-04 \\
2.56 \mathrm{E}-03 \\
0.00 \mathrm{E}+00 \\
7.86 \mathrm{E}-11 \\
6.20 \mathrm{E}-04 \\
4.10 \mathrm{E}-04 \\
3.83 \mathrm{E}-09 \\
3.37 \mathrm{E}-08 \\
3.61 \mathrm{E}-06 \\
9.64 \mathrm{E}-06 \\
2.65 \mathrm{E}-03 \\
7.33 \mathrm{E}-06 \\
5.20 \mathrm{E}-11 \\
7.90 \mathrm{E}-04 \\
2.50 \mathrm{E}-08 \\
1.61 \mathrm{E}-03 \\
7.96 \mathrm{E}-04 \\
1.40 \mathrm{E}-07 \\
4.22 \mathrm{E}-08 \\
5.22 \mathrm{E}-04 \\
2.35 \mathrm{E}-04 \\
1.16 \mathrm{E}-12 \\
1.71 \mathrm{E}-03 \\
2.85 \mathrm{E}-03 \\
5.73 \mathrm{E}-07 \\
3.06 \mathrm{E}-07 \\
2.62 \mathrm{E}-05 \\
1.37 \mathrm{E}-04 \\
1.91 \mathrm{E}-07 \\
2.77 \mathrm{E}-04 \\
5.91 \mathrm{E}-07\end{array}$ & $\begin{array}{l}1.43 \\
1.37 \\
1.78 \\
1.47 \\
1.37 \\
2.40 \\
1.38 \\
1.68 \\
2.10 \\
1.97 \\
1.71 \\
1.67 \\
1.35 \\
1.54 \\
2.42 \\
1.45 \\
2.66 \\
1.45 \\
1.46 \\
2.66 \\
1.95 \\
1.54 \\
1.52 \\
2.66 \\
1.50 \\
1.43 \\
2.52 \\
1.85 \\
1.45 \\
1.55 \\
1.87 \\
1.60 \\
2.46\end{array}$ & $\begin{array}{l}0.00 \mathrm{E}+00 \\
0.00 \mathrm{E}+00 \\
7.92 \mathrm{E}-03 \\
2.42 \mathrm{E}-04 \\
7.78 \mathrm{E}-03 \\
2.80 \mathrm{E}-04 \\
1.14 \mathrm{E}-02 \\
2.93 \mathrm{E}-02 \\
0.00 \mathrm{E}+00 \\
1.19 \mathrm{E}-03 \\
2.76 \mathrm{E}-03 \\
0.00 \mathrm{E}+00 \\
0.00 \mathrm{E}+00 \\
0.00 \mathrm{E}+00 \\
3.26 \mathrm{E}-03 \\
8.21 \mathrm{E}-05 \\
1.12 \mathrm{E}-03 \\
2.01 \mathrm{E}-03 \\
0.00 \mathrm{E}+00 \\
0.00 \mathrm{E}+00 \\
5.55 \mathrm{E}-05 \\
1.28 \mathrm{E}-04 \\
1.42 \mathrm{E}-04 \\
1.29 \mathrm{E}-03 \\
3.85 \mathrm{E}-02 \\
8.05 \mathrm{E}-02 \\
3.68 \mathrm{E}-05 \\
4.04 \mathrm{E}-02 \\
0.00 \mathrm{E}+00 \\
3.12 \mathrm{E}-03 \\
0.00 \mathrm{E}+00 \\
4.76 \mathrm{E}-03 \\
2.68 \mathrm{E}-04 \\
0.00 \mathrm{E}+00 \\
1.54 \mathrm{E}-03 \\
1.15 \mathrm{E}-03 \\
1.25 \mathrm{E}-01 \\
0.00 \mathrm{E}+00 \\
4.33 \mathrm{E}-03 \\
3.26 \mathrm{E}-03 \\
0.00 \mathrm{E}+00 \\
7.15 \mathrm{E}-03 \\
4.23 \mathrm{E}-02 \\
5.99 \mathrm{E}-02 \\
5.08 \mathrm{E}-03 \\
6.60 \mathrm{E}-03 \\
0.00 \mathrm{E}+00\end{array}$ & $\begin{array}{l}0.00 E+00 \\
0.00 E+00 \\
0.00 E+00 \\
0.00 E+00 \\
0.00 E+00 \\
0.00 E+00 \\
0.00 E+00 \\
0.00 E+00 \\
0.00 E+00 \\
0.00 E+00 \\
0.00 E+00 \\
0.00 E+00 \\
0.00 E+00 \\
0.00 E+00 \\
0.00 E+00 \\
0.00 E+00 \\
0.00 E+00 \\
0.00 E+00 \\
0.00 E+00 \\
0.00 E+00 \\
0.00 E+00 \\
0.00 E+00 \\
0.00 E+00 \\
0.00 E+00 \\
0.00 E+00 \\
0.00 E+00 \\
0.00 E+00 \\
0.00 E+00 \\
0.00 E+00 \\
0.00 E+00 \\
0.00 E+00 \\
0.00 E+00 \\
0.00 E+00 \\
0.00 E+00 \\
0.00 E+00 \\
0.00 E+00 \\
0.00 E+00 \\
0.00 E+00 \\
0.00 E+00 \\
0.00 E+00 \\
0.00 E+00 \\
0.00 E+00 \\
0.00 E+00 \\
0.00 E+00 \\
0.00 E+00 \\
0.00 E+00 \\
0.00 E+00\end{array}$ \\
\hline
\end{tabular}


Table B-2. R/rem Ratios and Detection Fractions, Continued

\begin{tabular}{|c|c|c|c|c|c|}
\hline Nuclide & $\begin{array}{l}\text { Results } \\
\text { mrem/h }\end{array}$ & $\begin{array}{c}\text { per } p^{p C i / g} \\
m R / h\end{array}$ & $\begin{array}{l}\text { R/rem } \\
\text { Ratio }\end{array}$ & $\begin{array}{l}\text { Detection, } \\
\text { GM P-11 }\end{array}$ & $\begin{array}{l}\mathrm{g} / \mathrm{cm}^{2} \\
\text { PAM }\end{array}$ \\
\hline 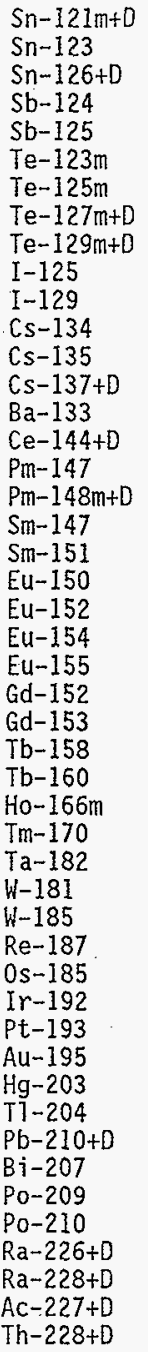 & $\begin{array}{l}1.39 E-06 \\
7.10 \mathrm{E}-06 \\
1.36 \mathrm{E}-03 \\
1.41 \mathrm{E}-03 \\
2.90 \mathrm{E}-04 \\
7.96 \mathrm{E}-05 \\
1.09 \mathrm{E}-06 \\
4.06 \mathrm{E}-06 \\
4.70 \mathrm{E}-05 \\
1.06 \mathrm{E}-06 \\
1.17 \mathrm{E}-06 \\
1.07 \mathrm{E}-03 \\
5.96 \mathrm{E}-09 \\
4.02 \mathrm{E}-04 \\
2.22 \mathrm{E}-04 \\
4.43 \mathrm{E}-05 \\
1.02 \mathrm{E}-08 \\
1.47 \mathrm{E}-03 \\
0.00 \mathrm{E}+00 \\
1.57 \mathrm{E}-10 \\
1.04 \mathrm{E}-03 \\
8.55 \mathrm{E}-04 \\
9.36 \mathrm{E}-04 \\
2.76 \mathrm{E}-05 \\
0.00 \mathrm{E}+00 \\
3.81 \mathrm{E}-05 \\
5.70 \mathrm{E}-04 \\
7.92 \mathrm{E}-04 \\
1.08 \mathrm{E}-03 \\
2.62 \mathrm{E}-06 \\
9.41 \mathrm{E}-04 \\
1.29 \mathrm{E}-05 \\
8.28 \mathrm{E}-08 \\
0.00 \mathrm{E}+00 \\
4.95 \mathrm{E}-04 \\
5.47 \mathrm{E}-04 \\
0.00 \mathrm{E}+00 \\
3.45 \mathrm{E}-05 \\
1.56 \mathrm{E}-04 \\
7.16 \mathrm{E}-07 \\
1.22 \mathrm{E}-06 \\
1.08 \mathrm{E}-03 \\
2.33 \mathrm{E}-06 \\
5.80 \mathrm{E}-09 \\
1.31 \mathrm{E}-03 \\
6.73 \mathrm{E}-04 \\
2.51 \mathrm{E}-04 \\
1.20 \mathrm{E}-03\end{array}$ & $\begin{array}{l}3.25 E-06 \\
1.04 E-05 \\
2.06 E-03 \\
1.95 E-03 \\
4.46 E-04 \\
1.37 E-04 \\
2.53 E-06 \\
6.75 E-06 \\
7.15 E-05 \\
2.60 E-06 \\
2.76 E-06 \\
1.58 E-03 \\
1.21 E-08 \\
5.96 E-04 \\
3.67 E-04 \\
6.64 E-05 \\
2.01 E-08 \\
2.18 E-03 \\
0.00 E+00 \\
3.87 E-10 \\
1.57 E-03 \\
1.21 E-03 \\
1.32 E-03 \\
5.23 E-05 \\
0.00 E+00 \\
7.32 E-05 \\
8.09 E-04 \\
1.13 E-03 \\
1.63 E-03 \\
5.02 E-06 \\
1.32 E-03 \\
2.63 E-05 \\
1.54 E-07 \\
0.00 E+00 \\
7.39 E-04 \\
8.76 E-04 \\
0.00 E+00 \\
6.75 E-05 \\
2.59 E-04 \\
1.37 E-06 \\
2.27 E-06 \\
1.56 E-03 \\
3.53 E-06 \\
8.44 E-09 \\
1.84 E-03 \\
9.62 E-04 \\
4.17 E-04 \\
1.63 E-03\end{array}$ & $\begin{array}{l}2.46 \\
1.51 \\
1.42 \\
1.41 \\
1.89 \\
1.92 \\
1.42 \\
1.43 \\
1.51 \\
1.92 \\
1.40 \\
2.04 \\
1.86 \\
1.49 \\
1.60 \\
1.96 \\
1.66 \\
1.91 \\
1.86 \\
1.44 \\
1.52 \\
1.46 \\
1.40 \\
1.43 \\
1.66 \\
1.36\end{array}$ & $\begin{array}{l}7.52 \mathrm{E}-04 \\
3.35 \mathrm{E}-02 \\
4.61 \mathrm{E}-02 \\
2.47 \mathrm{E}-02 \\
2.18 \mathrm{E}-03 \\
7.06 \mathrm{E}-04 \\
7.93 \mathrm{E}-04 \\
1.00 \mathrm{E}-02 \\
3.66 \mathrm{E}-02 \\
0.00 \mathrm{E}+00 \\
1.52 \mathrm{E}-04 \\
6.61 \mathrm{E}-03 \\
5.12 \mathrm{E}-04 \\
1.04 \mathrm{E}-02 \\
3.76 \mathrm{E}-04 \\
1.05 \mathrm{E}-01 \\
6.88 \mathrm{E}-04 \\
8.37 \mathrm{E}-03 \\
0.00 \mathrm{E}+00 \\
4.31 \mathrm{E}-09 \\
0.00 \mathrm{E}+00 \\
5.13 \mathrm{E}-03 \\
1.20 \mathrm{E}-02 \\
3.01 \mathrm{E}-04 \\
0.00 \mathrm{E} \div 00 \\
7.53 \mathrm{E}-05 \\
2.63 \mathrm{E}-03 \\
1.07 \mathrm{E}-02 \\
2.81 \mathrm{E}-03 \\
1.67 \mathrm{E}-02 \\
4.27 \mathrm{E}-03 \\
0.00 \mathrm{E}+00 \\
3.64 \mathrm{E}-03 \\
0.00 \mathrm{E}+00 \\
3.56 \mathrm{E}-04 \\
8.45 \mathrm{E}-03 \\
0.00 \mathrm{E}+00 \\
1.10 \mathrm{E}-04 \\
2.28 \mathrm{E}-03 \\
1.07 \mathrm{E}-02 \\
2.29 \mathrm{E}-02 \\
7.97 \mathrm{E}-03 \\
6.52 \mathrm{E}-06 \\
0.00 \mathrm{E}+00 \\
5.99 \mathrm{E}-02 \\
2.29 \mathrm{E}-02 \\
6.38 \mathrm{E}-02 \\
5.46 \mathrm{E}-02\end{array}$ & $\begin{array}{l}0.00 E+00 \\
0.00 E+00 \\
0.00 E+00 \\
0.00 E+00 \\
0.00 E+00 \\
0.00 E+00 \\
0.00 E+00 \\
0.00 E+00 \\
0.00 E+00 \\
0.00 E+00 \\
0.00 E+00 \\
0.00 E+00 \\
0.00 E+00 \\
0.00 E+00 \\
0.00 E+00 \\
0.00 E+00 \\
0.00 E+00 \\
0.00 E+00 \\
0.00 E+00 \\
0.00 E+00 \\
0.00 E+00 \\
0.00 E+00 \\
0.00 E+00 \\
0.00 E+00 \\
0.00 E+00 \\
0.00 E+00 \\
0.00 E+00 \\
0.00 E+00 \\
0.00 E+00 \\
0.00 E+00 \\
0.00 E+00 \\
0.00 E+00 \\
0.00 E+00 \\
0.00 E+00 \\
0.00 E+00 \\
0.00 E+00 \\
0.00 E+00 \\
0.00 E+00 \\
0.00 E+00 \\
0.00 E+00 \\
0.00 E+00 \\
0.00 E+00 \\
4.63 E-04 \\
5.84 E-04 \\
3.25 E-03 \\
0.00 E+00 \\
4.78 E-03 \\
4.71 E-03\end{array}$ \\
\hline
\end{tabular}


Table B-2. R/rem Ratios and Detection Fractions, Continued

\begin{tabular}{|c|c|c|c|c|c|}
\hline Nuclide & $\begin{array}{l}\text { Results } \\
\text { mrem/h }\end{array}$ & $=\underset{m R / h}{p C i / g}$ & $\begin{array}{l}\text { R/rem } \\
\text { Ratio }\end{array}$ & $\begin{array}{l}\text { Detection, } \\
\text { GM P-11 }\end{array}$ & $\begin{array}{r}\mathrm{g} / \mathrm{cm}^{2} \\
\text { PAM }\end{array}$ \\
\hline $\begin{array}{l}\text { Th-229+D } \\
\text { Th-230 } \\
\text { Th-232 } \\
P a-231 \\
U-232 \\
U-233 \\
U-234 \\
U-235+D \\
U-236 \\
U-238+D \\
N p-236 \\
N p-237+D \\
P u-236 \\
P u-237 \\
P u-238 \\
P u-239 \\
P u-240 \\
P u-241+D \\
P u-242 \\
P u-244+D \\
A m-241 \\
A m-242 m+D \\
A m-243+D \\
C m-242 \\
\mathrm{Cm}-243 \\
\mathrm{Cm}-244 \\
\mathrm{Cm}-245 \\
\mathrm{Cm}-246 \\
\mathrm{Cm}-247+D \\
\mathrm{Cm}-248 \\
\mathrm{Cf}-250 \\
\mathrm{Cf}-252 \\
\mathrm{Es}-254+D\end{array}$ & $\begin{array}{l}2.05 \mathrm{E}-04 \\
1.71 \mathrm{E}-07 \\
6.78 \mathrm{E}-08 \\
1.88 \mathrm{E}-05 \\
1.06 \mathrm{E}-07 \\
1.22 \mathrm{E}-07 \\
4.16 \mathrm{E}-08 \\
1.04 \mathrm{E}-04 \\
2.98 \mathrm{E}-08 \\
1.88 \mathrm{E}-05 \\
7.27 \mathrm{E}-05 \\
1.43 \mathrm{E}-04 \\
1.65 \mathrm{E}-08 \\
2.57 \mathrm{E}-05 \\
1.39 \mathrm{E}-08 \\
3.61 \mathrm{E}-08 \\
8.93 \mathrm{E}-09 \\
2.57 \mathrm{E}-09 \\
1.19 \mathrm{E}-08 \\
2.42 \mathrm{E}-04 \\
6.52 \mathrm{E}-06 \\
9.38 \mathrm{E}-06 \\
1.23 \mathrm{E}-04 \\
1.06 \mathrm{E}-08 \\
7.67 \mathrm{E}-05 \\
8.36 \mathrm{E}-09 \\
3.86 \mathrm{E}-05 \\
4.74 \mathrm{E}-09 \\
2.17 \mathrm{E}-04 \\
7.01 \mathrm{E}-09 \\
1.37 \mathrm{E}-08 \\
9.18 \mathrm{E}-09 \\
6.50 \mathrm{E}-04\end{array}$ & $\begin{array}{l}3.33 \mathrm{E}-04 \\
3.21 \mathrm{E}-07 \\
1.30 \mathrm{E}-07 \\
3.14 \mathrm{E}-05 \\
1.97 \mathrm{E}-07 \\
2.19 \mathrm{E}-07 \\
8.04 \mathrm{E}-08 \\
1.79 \mathrm{E}-04 \\
6.06 \mathrm{E}-08 \\
2.99 \mathrm{E}-05 \\
1.30 \mathrm{E}-04 \\
2.42 \mathrm{E}-04 \\
3.52 \mathrm{E}-08 \\
4.73 \mathrm{E}-05 \\
3.13 \mathrm{E}-08 \\
6.31 \mathrm{E}-08 \\
1.88 \mathrm{E}-08 \\
4.63 \mathrm{E}-09 \\
2.67 \mathrm{E}-08 \\
3.57 \mathrm{E}-04 \\
1.34 \mathrm{E}-05 \\
1.64 \mathrm{E}-05 \\
2.20 \mathrm{E}-04 \\
2.42 \mathrm{E}-08 \\
1.33 \mathrm{E}-04 \\
1.94 \mathrm{E}-08 \\
6.96 \mathrm{E}-05 \\
1.20 \mathrm{E}-08 \\
3.52 \mathrm{E}-04 \\
1.60 \mathrm{E}-08 \\
2.85 \mathrm{E}-08 \\
2.00 \mathrm{E}-08 \\
9.14 \mathrm{E}-04\end{array}$ & $\begin{array}{l}1.62 \\
1.88 \\
1.92 \\
1.67 \\
1.85 \\
1.80 \\
1.93 \\
1.72 \\
2.03 \\
1.59 \\
1.79 \\
1.69 \\
2.13 \\
1.84 \\
2.25 \\
1.75 \\
2.11 \\
1.80 \\
2.24 \\
1.48 \\
2.06 \\
1.75 \\
1.79 \\
2.28 \\
1.73 \\
2.32 \\
1.80 \\
2.53 \\
1.62 \\
2.28 \\
2.08 \\
2.18 \\
1.41\end{array}$ & $\begin{array}{l}3.94 \mathrm{E}-02 \\
0.00 \mathrm{E}+00 \\
1.57 \mathrm{E}-06 \\
2.07 \mathrm{E}-04 \\
2.81 \mathrm{E}-06 \\
2.91 \mathrm{E}-07 \\
1.69 \mathrm{E}-06 \\
1.49 \mathrm{E}-03 \\
1.30 \mathrm{E}-06 \\
6.51 \mathrm{E}-02 \\
2.26 \mathrm{E}-03 \\
4.46 \mathrm{E}-03 \\
0.00 \mathrm{E}+00 \\
4.61 \mathrm{E}-07 \\
0.00 \mathrm{E}+00 \\
0.00 \mathrm{E}+00 \\
0.00 \mathrm{E}+00 \\
5.57 \mathrm{E}-08 \\
0.00 \mathrm{E}+00 \\
4.62 \mathrm{E}-02 \\
1.27 \mathrm{E}-06 \\
6.41 \mathrm{E}-03 \\
5.41 \mathrm{E}-03 \\
0.00 \mathrm{E}+00 \\
2.20 \mathrm{E}-03 \\
0.00 \mathrm{E}+00 \\
7.67 \mathrm{E}-04 \\
0.00 \mathrm{E}+00 \\
6.15 \mathrm{E}-03 \\
0.00 \mathrm{E}+00 \\
1.31 \mathrm{E}-07 \\
1.88 \mathrm{E}-07 \\
1.45 \mathrm{E}-02\end{array}$ & $\begin{array}{l}4.87 \mathrm{E}-03 \\
4.05 \mathrm{E}-04 \\
2.31 \mathrm{E}-04 \\
5.23 \mathrm{E}-04 \\
5.84 \mathrm{E}-04 \\
4.46 \mathrm{E}-04 \\
4.31 \mathrm{E}-04 \\
3.29 \mathrm{E}-04 \\
3.54 \mathrm{E}-04 \\
2.78 \mathrm{E}-04 \\
0.00 \mathrm{E}+00 \\
4.32 \mathrm{E}-04 \\
7.19 \mathrm{E}-04 \\
0.00 \mathrm{E}+00 \\
6.36 \mathrm{E}-04 \\
5.39 \mathrm{E}-04 \\
5.42 \mathrm{E}-04 \\
0.00 \mathrm{E}+00 \\
4.69 \mathrm{E}-04 \\
3.81 \mathrm{E}-04 \\
6.33 \mathrm{E}-04 \\
2.66 \mathrm{E}-06 \\
5.74 \mathrm{E}-04 \\
8.29 \mathrm{E}-04 \\
7.39 \mathrm{E}-04 \\
7.31 \mathrm{E}-04 \\
6.00 \mathrm{E}-04 \\
6.04 \mathrm{E}-04 \\
4.83 \mathrm{E}-04 \\
4.75 \mathrm{E}-04 \\
8.02 \mathrm{E}-04 \\
8.07 \mathrm{E}-04 \\
9.31 \mathrm{E}-04\end{array}$ \\
\hline $\begin{array}{ll}\text { Zr-95 } & \text { old } \\
\text { Sb-125 old } \\
\text { Pb-210 old } \\
\text { Ra-226 old } \\
\text { Th-Nat } \\
\text { U-Nat } \\
\text { Pu } 6 \% \\
\text { Pu } 6 \% & 10 y r\end{array}$ & $\begin{array}{l}1.75 \mathrm{E}-03 \\
2.90 \mathrm{E}-04 \\
1.23 \mathrm{E}-06 \\
1.31 \mathrm{E}-03 \\
1.87 \mathrm{E}-03 \\
1.33 \mathrm{E}-03 \\
4.74 \mathrm{E}-07 \\
9.68 \mathrm{E}-07\end{array}$ & $\begin{array}{l}2.55 E-03 \\
4.47 E-04 \\
2.28 E-06 \\
1.84 E-03 \\
2.59 E-03 \\
1.87 E-03 \\
9.62 E-07 \\
1.98 E-06\end{array}$ & $\begin{array}{l}1.46 \\
1.54 \\
1.86 \\
1.41 \\
1.38 \\
1.41 \\
2.03 \\
2.05\end{array}$ & $\begin{array}{l}3.71 \mathrm{E}-03 \\
2.37 \mathrm{E}-03 \\
2.29 \mathrm{E}-02 \\
8.31 \mathrm{E}-02 \\
7.75 \mathrm{E}-02 \\
1.48 \mathrm{E}-01 \\
4.84 \mathrm{E}-07 \\
4.10 \mathrm{E}-07\end{array}$ & $\begin{array}{l}0.00 E+00 \\
0.00 E+00 \\
5.94 E-04 \\
3.84 E-03 \\
4.94 E-03 \\
4.95 E-03 \\
5.52 E-04 \\
5.58 E-04\end{array}$ \\
\hline
\end{tabular}




\section{MONO-ENERGETIC CHARGED PARTICLE READINGS}

Examples of mono-energetic charged particles are alphas, internal conversion electrons, and Auger electrons. They travel a given distance in matter (on the average) and then stop. The distance they travel depends on their initial kinetic energy.

The model for estimating instrument response begins with the radiation source uniformiy distributed in soil, or some other reasonably dense, low atomic number material such as water. In order for a charged particle to be detected, it must have enough energy to pass through the soil, the air between the soil and the detector, and finaliy the window of the detector. The window thickness on the PAM is $0.5 \mathrm{mg} / \mathrm{cm}^{2}$, while the window of the $G M$ pancake probe is about $3 \mathrm{mg} / \mathrm{cm}^{2}$. The density of air is such that each centimeter of thickness provides about $1.2 \mathrm{mg} / \mathrm{cm}^{2}$ of shielding material.

The total shielding between the PAM and the soil is assumed to be about $2 \mathrm{mg} / \mathrm{cm}^{2}$, based on an air thickness of about $1.7 \mathrm{~cm}$. The shielding between the GM and the soiT is assumed to be about $8 \mathrm{mg} / \mathrm{cm}^{2}$ based on an air thickness of about $6.7 \mathrm{~cm}$.

The total thickness that a charged particle can travel in low atomic number material was estimated from graphs found in the Radiological Health Handbook (HEW 1970) pages 123 and 125. One starts with a particular energy particle, such as the $5.15 \mathrm{MeV}$ aipha particie emitted by Pu-239. From the graph, the a]pha can penetrate $3.65 \mathrm{~cm}$ in air at STP, which corresponds to $4.7 . \mathrm{mg} / \mathrm{cm}^{2}$. Subtracting $2 \mathrm{mg} / \mathrm{cm}^{2}$ for the air thickness and detector window means that alphas from a depth of $2.7 \mathrm{mg} / \mathrm{cm}^{2}$ could reach the detector and be counted.

This needs to be adjusted for the detection geometry. The alphas are sent out from the source into all directions, but only certain directions will reach the detector. Due to the proximity of the soil and detector, it is assumed that 20 percent of the alphas could be detected. The geometry factor is therefore 0.20 .

\section{Detection Factor $=\left(\right.$ Soii Depth, $\left.\mathrm{mg} / \mathrm{cm}^{2}\right)$ (Geometry Factor)}

The detection factor in this case is $5.39 \mathrm{E}-4 \mathrm{~g} / \mathrm{cm}^{2}$, which matches the value shown on Table B-2. Knowing the probe face area and the soil contamination, one can now estimate the number of particles reaching the detector. (It is assumed that every charged particle entering the active volume of the detector will be counted.)

This detection factor is put to use by multiplying it by the effective face area of the detector and the soil concentration. The result is the number of detected particles per unit time. For example, the PAM probe area is $50 \mathrm{~cm}^{2}$ and the $100 \mathrm{mrem} / \mathrm{y}$ soil limit for Pu-239 is $19.1 \mathrm{pCi} / \mathrm{g}$, so the estimated alpha detection rate is shown below (and a so on Table 3 ).

$$
\text { Detection Rate }=\left(5.39 \mathrm{E}-4 \mathrm{~g} / \mathrm{cm}^{2}\right)\left(50 \mathrm{~cm}^{2}\right)(19.1 \mathrm{pCi} / \mathrm{g})=0.51 \text { per minute }
$$

Since the instrument operator needs to hear a rate of at least 100 per minute to notice the contamination, this particular Pu-239 soil limit will probably go undetected with the PAM. However, the granular nature of soil may allow detection if the plutonium particles are on the upper surfaces of the grains. Converseiy, even higher levels could escape detection if the soil grains were 
covering the plutonium. Detecting alpha contamination in soil depends greatly on the condition of the soil.

The method for mono-energetic particles was automated with a computer program named DETECT which interpolates between values read from the graphs in the Handbook. The computer program uses the DRALIST nuclear data library (Kocher 1981) for the energies of the charged particles. The DETECT program is described in Appendix $C$.

\section{BETA AND POSITRON READINGS}

Beta decay and positron emission produce charged particles with energies ranging from zero up to a characteristic maximum, the endpoint energy for the decay. The same approach used for mono-energetic charged particles was extended to this case. The difference is that the detection factors for the various particle energies must be weighted by their probability and summed to calculate the overall detection factor.

Electrons and positrons emitted from a nucleus undergoing beta decay are produced with initial kinetic energies that range from zero to the endpoint energy, $E_{0}$. The probability of finding one of these particles with a total energy between $W$ and $(W+d W)$ is given by the equation below.

$$
\operatorname{Prob}(W) d W=K \text { Fermi }( \pm Z, W) \text { p W }\left(W_{0}-W\right)^{2} d W S_{x}
$$

The Fermi function for electrons $(+Z)$ and positrons $(-Z)$ is calculated from tabulated values as shown in the next equation.

$$
\operatorname{Fermi}( \pm Z, W)=K F_{N B S} / p^{2}
$$

The values of $F_{\text {NBS }}$ are listed in the National Bureau of Standards (NBS 1952) Mathematical Handbook. They were caiculated from the formula shown below. The DETECT program described in Appendix $C$ uses a data file which has the NBS values divided by the electron momentum. This combination is nearly linear with momentum, which ensures good accuracy when doing a simple linear interpolation between data points. The values for $F_{\text {BBS }}$ for positrons are simply the electron values times $\operatorname{EXP}(-2 \pi v)$.

$$
F_{N B S}=p^{2 g} \operatorname{Exp}(\pi v)|\Gamma(g+i v)|^{2}
$$

The Shape factor $\left(S_{x}\right)$ depends on the degree of "forbiddenness" of the particular decay mode. Shape factors for the first four degrees are shown below (Siegbahn 1968).

$$
\begin{aligned}
& S_{0}=1 \\
& S_{1}=p^{2}+\left(W_{0}-W\right)^{2} \\
& S_{2}=p^{4}+\left(W_{0}-W\right)^{4}+10 / 3 p^{2}\left(W_{0}-W\right)^{2} \\
& S_{3}=p^{6}+\left(W_{0}-W\right)^{6}+7 p^{2}\left(W_{0}-W\right)^{2}\left[p^{2}+\left(W_{0}-W\right)^{2}\right]
\end{aligned}
$$


The " $g$ " and " $v$ " variables used above are defined in the equation below.

$$
g=\sqrt{1-(\alpha Z)^{2}} \quad v=\frac{\alpha Z W}{p}
$$

The variables used in the above equations, as well as later ones are defined below.

$$
\begin{aligned}
& E=\text { beta particle kinetic energy, in } \mathrm{MeV} \\
& W=\text { total rest-mass energy, in units of } \mathrm{mc}^{2}, W=1+E /\left(\mathrm{mc}^{2}\right) \\
& \mathrm{p}=\text { beta particle momentum, } \mathrm{p}^{2}=W^{2}+1 \\
& \alpha=\text { fine structure constant }=1 / 137.036 \\
& Z=\text { atomic number of the daughter nucleus } \\
& X=\text { a normalization factor which ensures the total probability is } 1
\end{aligned}
$$

The energy spectra defined above can be used to compute the momentum spectra using the relation shown in equation 6 . Momentum spectra were frequently used to report beta spectra data measured in magnetic spectrometers since the product of the magnetic field strength and the radius of curvature is proportional to the particie momentum.

$$
\operatorname{Prob}(p) d p=\operatorname{Prob}(E) d E\left(m c^{2} p / W\right)
$$

The DETECT program reads table values for the Fermi function at electron momenta ranging from 0.01 to 20 in 420 steps of varying length. The units of electron momentum are $m c$, where $m$ is the mass of the electron and $c$ is the speed of light. The values for the Fermi function were computed by numerical integration of the complex gamma function and stored in a binary file. This was done as a check on the NBS values and to avoid transposition errors when copying numbers.

The surface soil is divided into thin layers and the minimum energy electron which can travel from each layer to the detector is computed. The beta probability function, Prob(W), is used to determine the fraction of the electrons from each layer which would be counted. This fraction is the integral of the beta probability function from the minimum energy to the maximum energy. These fractions were weighted by the thickness of the soil layer and summed to give the total detection fraction.

Results for the detection factor for each nuclide were shown in Table B-2. These were computed by the DETECT program described in Appendix C. 
Appendix C. The DETECT Program Version 2.0 


\title{
USER'S GUIDE TO DETECT
}

The purpose of the program is to estimate detection factors for one or more lists of nuclides. A list of nuclides is a text.file with individual nuclides or groups of nuclides which act together. The DETECT program reads through the nuclide list file and computes the detection factor for each nuclide and group of nuclides in the file. Scale factors may be applied to individual nuclides or groups of nuclides. Other inputs are the geometry factor, the initial air plus detector shielding thickness, and the thickness of the source. There is an option to calculate only alpha particle detection and omit electrons.

The files listed in Table C-1 are distributed as the DETECT software. The first six DRALIST files are the binary version of DRALIST (Kocher 1981) used by the DETECT program. These were created by the MAKE-DRA program. The SHOW-DRA program allows one to selectively display data in the DRALIST binary files to verify that it is correctly stored and read.

\section{Table C-1. Files Distributed as the DETECT Software}

\author{
FERMI.BIN - Binary file with Fermi function values. \\ DRALIST.N - DRALIST general data for each nuclide. \\ DRALIST.A - DRALIST alpha emissions for each nuclide. \\ DRALIST.B - DRALIST beta emissions for each nuclide. \\ ORALIST.P - DRALIST positron emissions for each nuclide. \\ DRALIST.E - DRALIST electron emissions for each nuclide. \\ DRALIST.G - DRALIST gamma photon emissions for each nuclide. \\ DRALIST.SUM - Data summary generated by MAKE-DRA.PAS. \\ MAKE-DRA.PAS - Pascal source code to read the DRALIST.DAT file and create the \\ DRALIST.? binary files. \\ SHOW-DRA.EXE - Program to display nuclide data in the binary files. \\ SHOW-DRA.PAS - Pascal source code for SHOW-DRA.EXE. \\ DETECT.EXE - The executable for the DETECT program. \\ DETECT.PAS - Pascal source code for DETECT.EXE. \\ DRALIST.PAS - Pascal unit to read the DRALIST files. \\ GENERAL.PAS - Pascal unit with general subroutines. \\ NUCLIST.PAS - Pascal unit to read nuclide files. \\ SRCFRAC.PAS - PasCaT unit to generate beta spectrum and integrals. \\ TEST-DET.BAT - Batch file to run the test cases and compare with valid output. \\ TEST.NUC - Nuclide file for the test cases. \\ TEST.N3 - Test case output $-3 \mathrm{mg} / \mathrm{cm}^{2}$ shielding and the default geometry \\ factor of 0.5 . \\ TEST.N5 - Test case output - default $\left(5 \mathrm{mg} / \mathrm{cm}^{2}\right)$ shielding and geometry \\ factor (0.5). \\ TEST.N9 - Test case output - $9 \mathrm{mg} / \mathrm{cm}^{2}$ shielding and the default geometry \\ factor $(0.5)$. \\ TEST.N8 - Test case output - $9 \mathrm{mg} / \mathrm{cm}^{2}$ shielding and geometry factor of \\ 0.25 . \\ TEST.NA - Test case output for alpha only, $3 \mathrm{mg} / \mathrm{cm}^{2}$ shielding with the \\ default geometry factor $(0.5)$.
}

The test cases are run using the TEST-DET.BAT file. This creates five output files named TEST.E3, TEST.E5, TEST.E9, TEST.E8, and TEST.EA for comparison with the verified test case output files shown above. 
The DETECT program is started using the command DETECT, at the DOS prompt. With no command Tine parameters, the program displays a summary of what it does and how it is run. This summary is shown in Table C-2 below.

Table C-2. Help Screen for DETECT

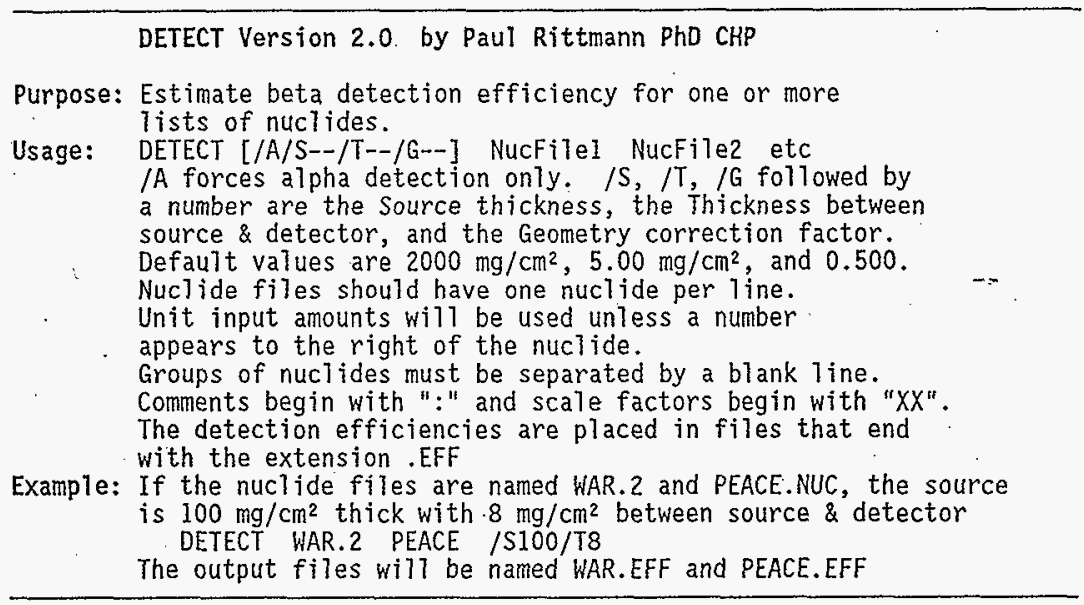

The command line controls the input to DETECT. There may be more than one file name on the command line. Typing the .Nuc extension is optional. The program first looks for the filename you have entered. If it is found, it is used, otherwise the .NUC extension is added and a second attempt is made to find the nuclide file. If this is not successfut, the program terminates with a message that the file in question could not be located.

Changes to the default soil thickness or geometry factor are indicated with the "/" character. One of the valid command options (A, S, T, or G) must immediately follow this character. The $S, T$ and $G$ options require a number. The A option must not have a number.

The TEST.NUC file shows the variety of input formats which are acceptable to the program. This input file is shown in Table $c-3$ below for reference. Line numbers have been added to facilitate a discussion of its contents.

Line 1 shows a comment. Lines beginning with non-aiphabetic characters are assumed to be comments and are skipped.

Line 2 shows that leading spaces are ignored. Trailing spaces are also ignored, as are spaces between the element and the isotopic mass, as in Line 6 .

The nuclide name can be in upper or lower case, the program makes the necessary conversions. In addition, the hyphen is optionat.

Line 14 shows the addition of an adjustment factor using a line beginning with "XX". All input values after this are multiplied by the number which appears 
on the scale factor line. Line 22 shows another use of the scale factor line. This one has no value to the right. Therefore, the value 1.0 is assumed. The Ra-226 group are a17 adjusted by the factor 0.00432 . However, Line 22 causes the Co-60 and other nuclides to be interpreted as having no adjustment (scale factor is 1.0$)$. Line 36 changes the scale factor to 2.0 , but the nuclides to which it would apply are fictitious. Line 43 returns the scale factor to 1.0 for doing $K-40$.

Table C-3. Contents of TEST.NUC with Line Numbers Added

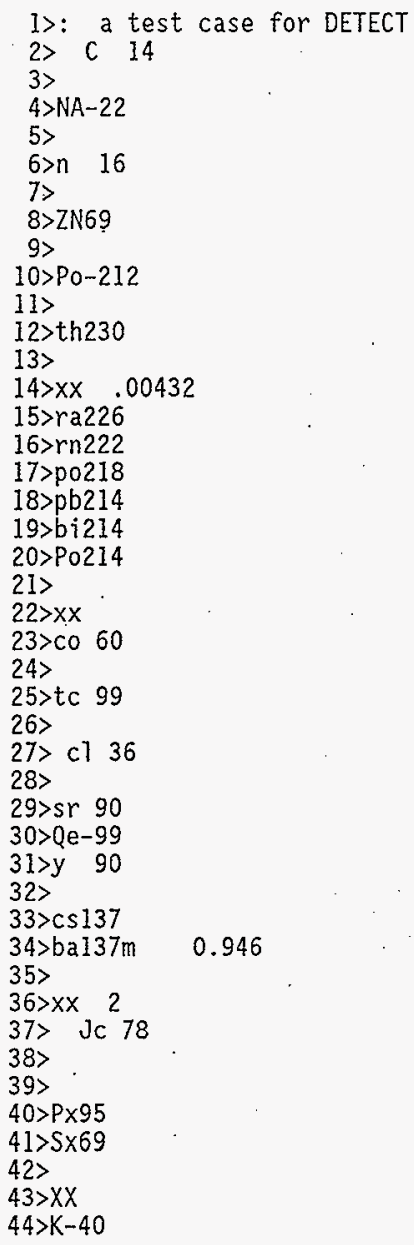


Line 34 shows how to include equilibrium factors for nuclides which are in secular equilibrium. Any number to the right of the nuclide name is interpreted as a second scale factor which only applies to that nuclide.

A11 output from DETECT is set to a file with the extension. EFF. The first part of the file name is the same as the nuclide list file. For example, output from TEST.NUC will be written to TEST.EFF. Since new output overwrites any existing output, the output file may need be copied to a file with a distinctive name to preserve it. This is the approach taken in the TEST-DET.BAT file to use the TEST.NUC file with various other inputs.

The output from DETECT is organized in two parts. The first part shows the adjusted amount for each nucijde, the detection factor and the type of particles contributing to that result. The notation is $a=a l p h a, b=b e t a$, $p=p o s i t r o n$, and e=electron. The second part shows the combined results for each nuclide or group of nuclides in the nuclide 7 ist. Groups of nuclides are indicated with a ".t" sign between the name of the first nuclide in the group and the resulting detection efficiency. The sample file named TEST.N5 is shown below to illustrate this.

Table C-4. Output from TEST.NUC Using the Program Defautts

Output from DETECT Version 2.0 by Paul Rittmann PhD CHP

Source Nuclide Groups and Detected Fractions:

Geometry Factor: 0.500

Initial Thickness: $5.00 \mathrm{mg} / \mathrm{cm}^{2}$

Source Thickness: $2000 \mathrm{mg} / \mathrm{cm}^{2}$

Minimum Alpha, Beta keV: $5361,57.0$ source to detector

Minimum Alpha, Beta keV: 1110499, 3949 from back of source

\begin{tabular}{|c|c|c|c|}
\hline Nuclides & $\begin{array}{l}\text { Input } \\
\text { Amount }\end{array}$ & $\begin{array}{l}\text { Fraction } \\
\text { gram } / \mathrm{cm}^{2}\end{array}$ & $\begin{array}{c}\text { Particle } \\
\text { Types }\end{array}$ \\
\hline $\begin{array}{l}\mathrm{C}-14 \\
\mathrm{Na}-22 \\
\mathrm{~N}-16 \\
\mathrm{Zn}-69 \\
\mathrm{Po}-212 \\
\mathrm{Th}-230 \\
\mathrm{Ra}-226 \\
\mathrm{Rn}-222 \\
\mathrm{Po}-218 \\
\mathrm{~Pb}-214 \\
\mathrm{Bi}-214 \\
\mathrm{Po}-214 \\
\mathrm{Co}-60 \\
\mathrm{Tc}-99 \\
\mathrm{C} \mathrm{C}-36 \\
\mathrm{Sr}-90 \\
\mathrm{Qe}-99 \\
\mathrm{Y}-90 \\
\mathrm{Cs}-137 \\
\mathrm{Ba}-137 \mathrm{~m} \\
\mathrm{Jc}-78\end{array}$ & $\begin{array}{l}1.000 E+00 \\
1.000 E+00 \\
1.000 E+00 \\
1.000 E+00 \\
1.000 E+00 \\
1.000 E+00 \\
4.320 E-03 \\
4.320 E-03 \\
4.320 E-03 \\
4.320 E-03 \\
4.320 E-03 \\
4.320 E-03 \\
1.000 E+00 \\
1.000 E+00 \\
1.000 E+00 \\
1.000 E+00 \\
1.000 E+00 \\
1.000 E+00 \\
1.000 E+00 \\
9.460 E-01 \\
2.000 E+00\end{array}$ & $\begin{array}{r}1.06 \mathrm{E}-03 \\
2.07 \mathrm{E}-02 \\
5.59 \mathrm{E}-01 \\
4.36 \mathrm{E}-02 \\
2.95 \mathrm{E}-03 \\
3.58 \mathrm{E}-05 \\
1.08 \mathrm{E}-06 \\
3.96 \mathrm{E}-07 \\
2.11 \mathrm{E}-06 \\
1.37 \mathrm{E}-04 \\
5.20 \mathrm{E}-04 \\
8.45 \mathrm{E}-06 \\
5.92 \mathrm{E}-03 \\
4.67 \mathrm{E}-03 \\
2.97 \mathrm{E}-02 \\
1.72 \mathrm{E}-02 \\
\text { Not Found!! } \\
1.87 \mathrm{E}-01 \\
1.72 \mathrm{E}-02 \\
1.00 \mathrm{E}-02 \\
\text { Not Found!! }\end{array}$ & $\begin{array}{l}b \\
p \\
b \\
b \\
a \\
e \\
e \\
a \\
a \\
b e \\
b e \\
a \\
b \\
b \\
b \\
b \\
b \\
b \\
e\end{array}$ \\
\hline
\end{tabular}




\begin{tabular}{|c|c|c|c|}
\hline $\begin{array}{l}P x-95 \\
S x-69 \\
K-40\end{array}$ & $\begin{array}{l}2.000 E+00 \\
2.000 E+00 \\
1.000 E+00\end{array}$ & $\begin{array}{r}\text { Not Found!! } \\
\text { Not Found!! } \\
7.45 \mathrm{E}-02\end{array}$ & $b$ \\
\hline
\end{tabular}

Estimated Detected Fractions, gram per $\mathrm{cm}^{2}$ :

Geometry Factor: 0.500

Initial Thickness: $5.00 \mathrm{mg} / \mathrm{cm}^{2}$

Source Thickness: $2000 \mathrm{mg} / \mathrm{cm}^{2}$

Minimum Alpha, Beta keV: $5361,57.0$ source to detector

Minimum Alpha, Beta keV: 1110499, 3949 from back of source

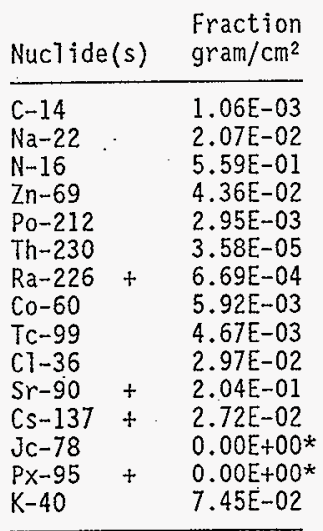

*NucTide not in DRALIST.

To compute the particle detection rate, multiply the table values by the probe area (in $\mathrm{cm}^{2}$ ) and the source concentration in units of activity per gram. 


\section{MODELLING DETAILS}

The DETECT program finds the range of a given energy particle (or the energy given the range) by interpolation on the values shown in Table $C-5$. The values were read from graphs in the Radiological Health Handbook (HEW 1970). Linear interpolation is used for alpha particies. The beta interpolation uses log scales for both energy and range. The minimum allowed initial thickness was chosen to be $1 \mathrm{mg} / \mathrm{cm}^{2}$.

Table C-5. Range in Air for Various Aipha and Electron Energies

\begin{tabular}{|c|c|c|}
\hline \multicolumn{3}{|c|}{ Alpha Particles } \\
\hline $\begin{array}{l}\text { Range } \\
\mathrm{cm} \text { air }\end{array}$ & $\begin{array}{l}\text { Range } \\
\mathrm{mg} / \mathrm{cm}^{2}\end{array}$ & $\begin{array}{c}\text { Energy } \\
\mathrm{keV}\end{array}$ \\
\hline 0.50 & 0.647 & 1000 \\
\hline 0.74 & 0.957 & 1500 \\
\hline 1.00 & 1.293 & 2000 \\
\hline 1.29 & 1.668 & 2500 \\
\hline 1.63 & 2.108 & 3000 \\
\hline 2.01 & 2.599 & 3500 \\
\hline 2.44 & 3.155 & 4000 \\
\hline 2.93 & 3.788 & 4500 \\
\hline 3.47 & 4.487 & 5000 \\
\hline 4.02 & 5.198 & 5500 \\
\hline 4.62 & 5.974 & 6000 \\
\hline 5.26 & 6.801 & 6500 \\
\hline 5.95 & 7.693 & 7000 \\
\hline 6.63 & 8.573 & 7500 \\
\hline 7.33 & 9. & 8000 \\
\hline
\end{tabular}

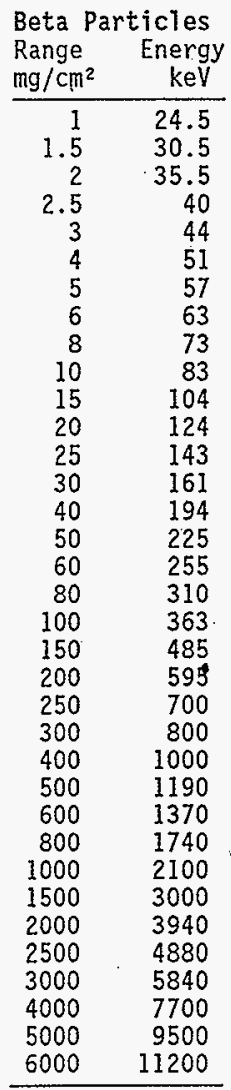

Numeric integration of the beta spectrum is carried out using Simpson's Rule. When the spectrum is computed from the endpoint energy, the nuclear transition is assumed to have a forbiddenness of 0 , which tends to underestimate the 
detection factor. (Larger values for forbiddenness lead to larger detection factors.) The spectrum is computed at 500 equally spaced points. This large number is selected for two reasons. First, it ensures adequate accuracy near the endpoint energy where the probability function decreases rapidly with energy. Second, it allows a simplification during the numeric integration with soil depth to find the detection factor. At a given soil depth there is a minimum electron energy which is able to reach the detector. The beta integration begins at this energy and ends at the endpoint energy of the beta. To simplify the integration, it is assumed to begin at the next larger energy in this particle's probability function. Having a large number of points means that the small amount of area that is ignored will have no effect on the final answer.

The integration over soil depth is accomplished using the trapezoid rule on layers that increase exponentialiy in thickness. The first soil thickness is zero so that the beta particle range is the initial shield thickness. . The second thickness is the first thickness multiplied by the factor shown below.

$$
\text { Thickness Factor }=\left|\frac{6000 \mathrm{mg} / \mathrm{cm}^{2}}{\text { Initial Shielding }}\right|^{0.005}
$$

The thickness factor is computed so that after 200 applications of the factor, the range will be the maximum allowable range, $6000 \mathrm{mg} / \mathrm{cm}^{2}$. Since the maximum beta energy in the DRALIST. library is $10.419 \mathrm{MeV}(\mathrm{N}-16)$, no beta will ever exceed a range of $6000 \mathrm{mg} / \mathrm{cm}^{2}$.

Since each successive thickness is this factor times the preceding thickness, integration by the trapezoid rute uses the equation shown below.

$$
\begin{aligned}
\text { Integral } & =\sum_{K=1}^{N-1}\left(P_{K}+P_{K+1}\right)\left(X_{K+1}-X_{K}\right) / 2 \\
& =P_{1} X_{1}(f-1) / 2+P_{K} X_{N}(1-1 / f) / 2+\sum_{K=2}^{N-1} P_{K} X_{K}(f-1 / f)
\end{aligned}
$$

where,

$N=$ Number of points to reach zero penetration.

$P=$ Probability function for the beta emission.

$X=$ Thickness of shielding between this layer and the detector.

$f=$ Thickness factor defined above. 


\section{PROGRAM LISTING - DETECT.PAS}

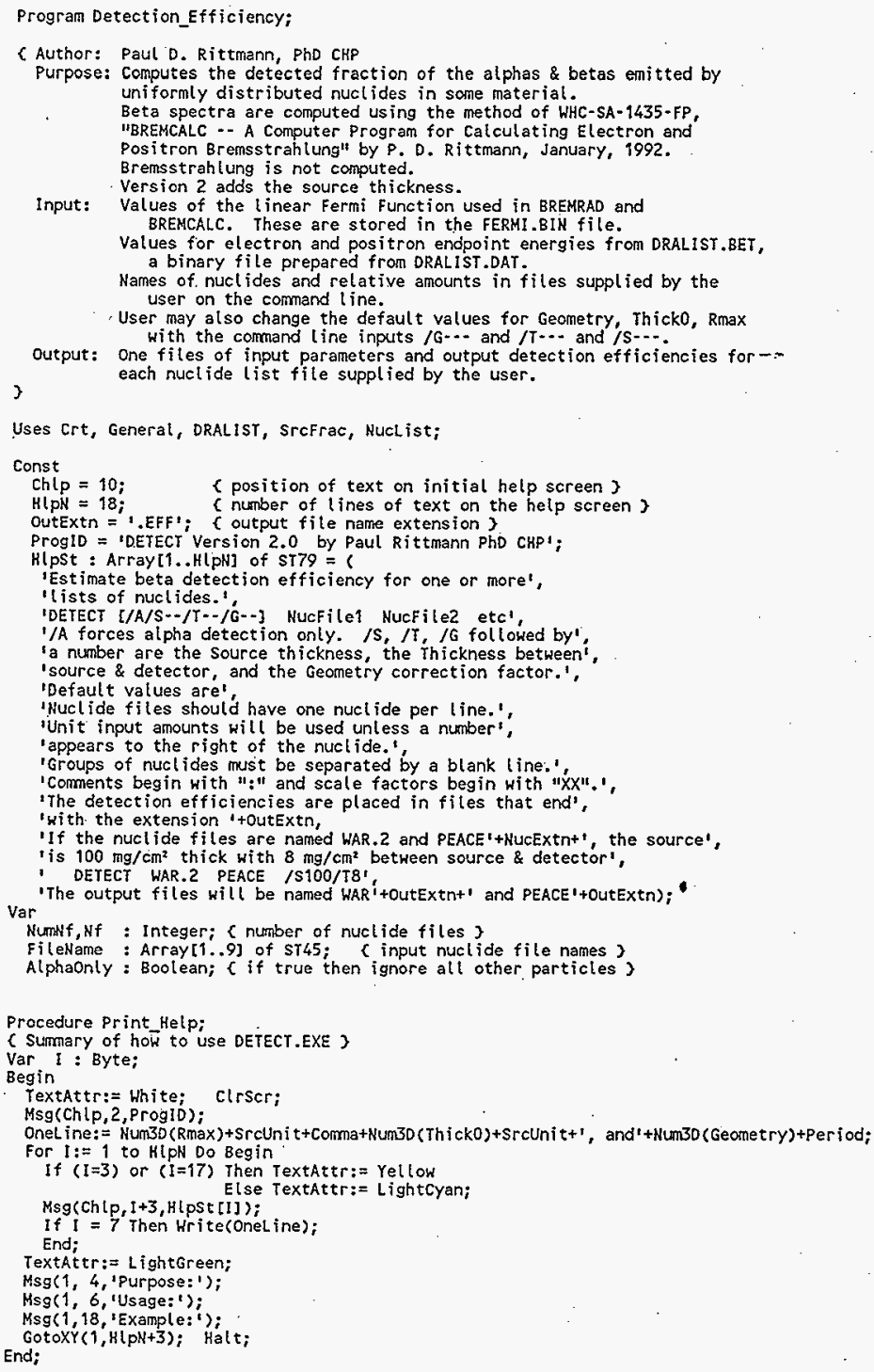


Procedure Check_Comand_Line;

- Reads the fil $\vec{e}$ names on the comnand line and looks for them.

Also interprets changes in the initial thickness or geonetry factor. $)$ Var $\mathrm{CH}$ : Char:

$I, K, J$,

Lng, $N$ : Byte:

PST : ST79;

('string indices and file counter )

fi : Text;

(string length and comand line parameter index \}

Procedure Quit_Msg(Msg:S17.9);

(Uses STo for the final message and halts execution.

Displays the comand line parameter which caused the error. )

Begin

TextAttr:= LightMagenta;

Writeln i $\star * * *$ 'Msg,' ', PST);

Writeln' TYpe DEIECT for guidance on how to use this progran. ');

Halt;

End;

Begin

Numkf: = ParamCount

If NunNf $=0$ Then Print Help;

$J:=0 ; \quad$ Alphaonly:= fälse; TextAttr:= Yellow;

For $N:=1$ to Numinf Do Begin

PST: $=$ Parainstr $(N) ; \quad$ Lng: $=$ Length(PST);

CH: = PST [Lng] ;

If $(\mathrm{CH}=1 / 1$ ) or $(\mathrm{CH}=1,1)$ then $\operatorname{Dec}(\mathrm{Lng})$.

If Lng $=0$ Then Quit Msg('Invalid comand (ine input: ');

For $K:=1$ to Lng Do OneL ine $[K]:=$ UPCase(PST $[K]$ ); OneL ine $[0]:=\operatorname{Chr}(L n g)$ :

If (PST[1] $=1 / 1$ ) and (L.ng $>1$ ) Then Begin ( read one or more flags

$K:=1 ;$

Repeat ( loop starts with $K$ positioned at the first / in ST

Inc $(K) ; \quad C H:=$ OneL ine $[K]$;

If $\mathrm{CH}=$ 'A' Then AlphaOniy: = true Else

If $\left(\mathrm{CH}=\mathrm{I}^{\prime}\right)$ or $\left(\mathrm{CH}=\mathrm{IS}^{\prime}\right)$ or $\left(\mathrm{CH}=\mathrm{G}^{\prime}\right)$ Then Begin

$\operatorname{Inc}(K)$;

If $K>$ ' Lng Then Quit Msg('No number supplied in option string: '):

$I:=K$;

While (oneline $[K]<>1 / 1)$ and $(K<L n g)$ Do Inc $(K)$;

If Onel ine $[K]=1 / 1$ then $\operatorname{Dec}(K)$;

If Valid Number ( $I, K$, factor)

Then IF $\mathrm{CH}=$ 'G' then Geometry:= Factor $E$ ise

If $\mathrm{CH}=$ ' $\mathrm{S}$ ' Then Rmax:= Factor

Etse Thick $0:=$ Factor

End

Else Quit Msg(' Invalid number supplied in option string: ');

Else Quit Msg('Invalid progran option:');

Inc $(K)$;

Until $K>$ Lng;

If ThickO $<1$ Then Begin PST: $\approx 11$

Quit_Msg('initial shielding must be greater than $1 \mathrm{mg} / \mathrm{cm}^{2} 1 \mathrm{II}^{1}$ ); End

Else Begin ( nuclide file name $\}$

Assign( $f i$ Oneline); $\{\$ I-\}$ Reset $(f i) ;\{\$ I+\}$

If IOResult $>0$

Then Begin

Onel ine:= Onel ine + NucExtn:

Assign(fi, OneLine); $(\$ I-\} \operatorname{Reset}(f i) ;\langle \$ I+\rangle$

If ioresult $\rightarrow 0$

Then Quit Msg('Nuclide fille could not be found:')

End

Else Close(fi);

Inc(J); fileName [J]:= OneL ine;

End

End;

If $J>0$ Then NunN $:=J$

Else Begin PST:= '';

Quit_Msg('No nuclide files were provided! ' ');

End; End;

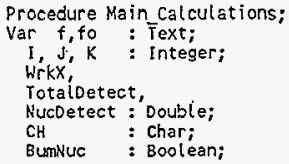




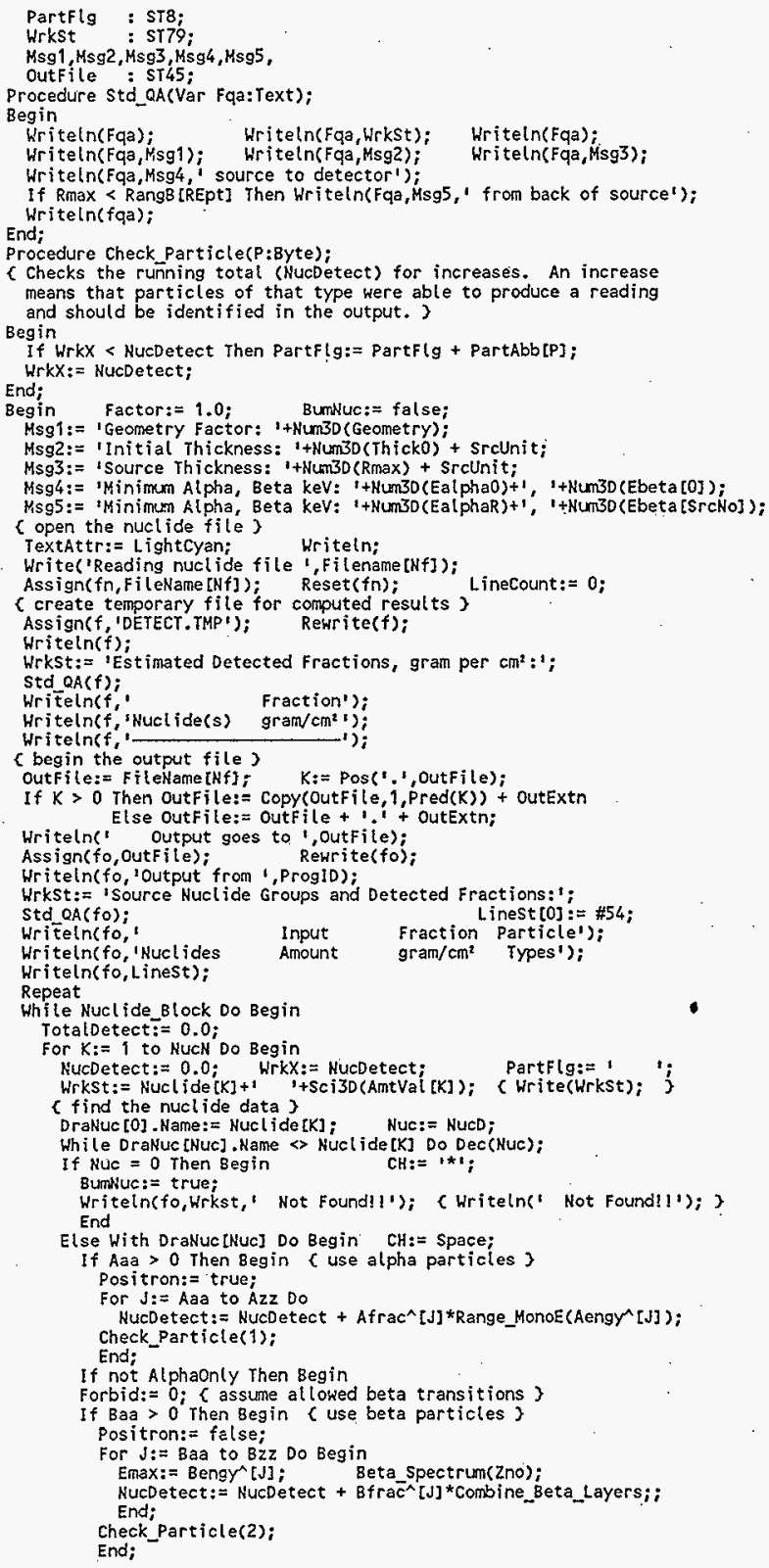


If Paa > 0 Then Begin \{ use positrons )

Positron: $=$ true;

For $f:=$ Paa to Pzz Do segin

Emax: $=$ Pengy $^{\wedge}[\sqrt{ }]$;

Beta_Spectrum(Zno)

NucDetect $:=$ NucDetect + Pfrac^ $[J]^{\star}$ Combine_Beta_Layers; ; End;

Check_Particle(3):

End;

If Eaa > 0 Then Begin ( use Auger \& IC electrons )

Positron: = false;

For J:= Eaa to Ezz Do

NucDetect: $=$ NucDetect $+E_{\text {frac }}^{\wedge}[\mathrm{J}] *$ Range_MonoE (Eengy^ $[\mathrm{J}]$ ); Check Particle(4):

End;

End;

NucDetect: = NucDetect*AntVal $[K]{ }^{*}$ Geometry;

Msg1:= : 'Sci20 (NucDetect);

Writeln(fo, Hrkst, Msg?, PartF(g);

End:

TotalDetect: $=$ TotalDetect + NucDetect;

End; $\{$ of loop over nuclides in a group

If Nuck > 1 Then Wrkst: $=1+1$ Else Wrkst: $=1$,

Writelns, Nucl ide[1], Hrkst, Sci2D(TotalDetect), CH);

End;

Untit Eof(fn):

Writeln(fo, Linest).

Writelncf,

i):

close $(f n)$;

If Bumuc Then Writeln(f, "Nuclide not in DRALIST. '); Reset(f);

While not Eof( $f$ ) Do Begin ( append the temporary file to the output )

Readln(f, Oneline); Writeln(fo, Oneline); End;

Writeln(fo);

Writeln(fo, 'To compute the particle detection rate, multiply the');

Hriteln(fo,' table values by the probe area (in $\mathrm{cm}^{2}$ ) and the source');

Writeln(fo, 'concentration in units of activity per gratn. "): End;

close(fo); close(f); $\operatorname{Erase}(f)$;

Begin

check_Command_Linge;

Init Ferni :

If ATphaonly Then Get oralist $(1,0,0,0,0)$

Else Get_DRALIST $(1,1,1,1,0)$;

For Nf:= 1 to Numf Do Main Calculations:

Close(FF):

[ loop through nuclide files End. 


\section{PROGRAM LISTING - GENERAL.PAS}

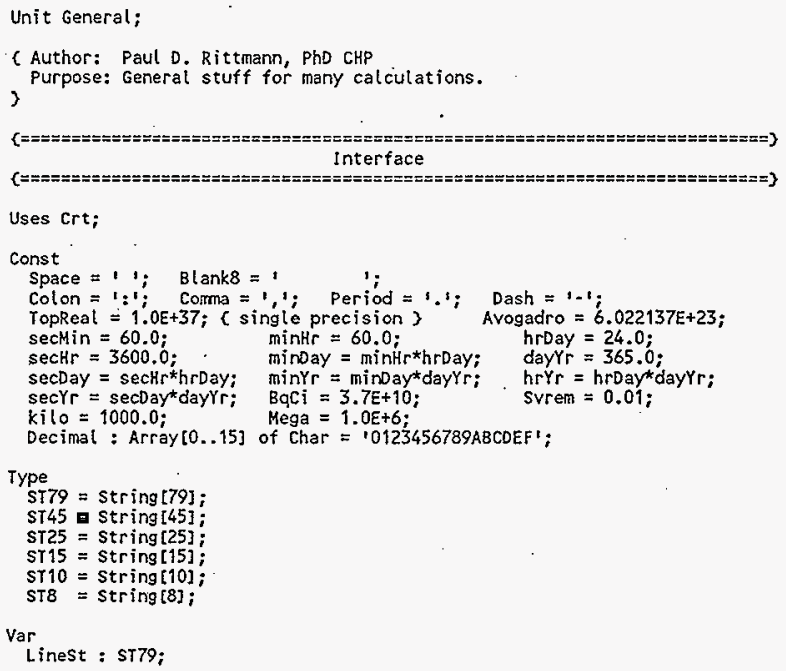

Procedure Msg(X,Y:Integer; STR : ST79); $\{$ displays STR at $X, Y$ on the screen $\}$ Begin GotoXY $(X, Y)$; Write(STR) End;

Function Num2S(X:Single) : $\$ T 10_{i}$ (Returns a string with $1 \mathrm{E}-6<X<1 E 9$ showing 2 significant digits.) Var Wrkst : string [11]; 


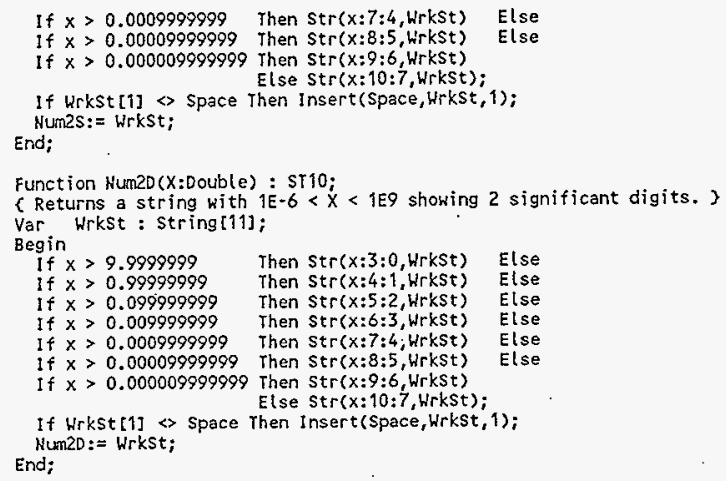




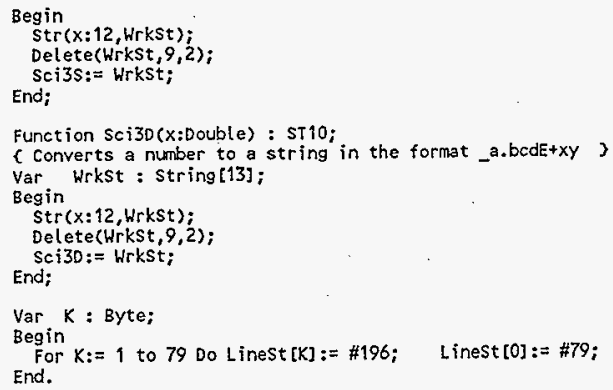




\section{PROGRAM LISTING - DRALIST.PAS}

Unit DRALIST;

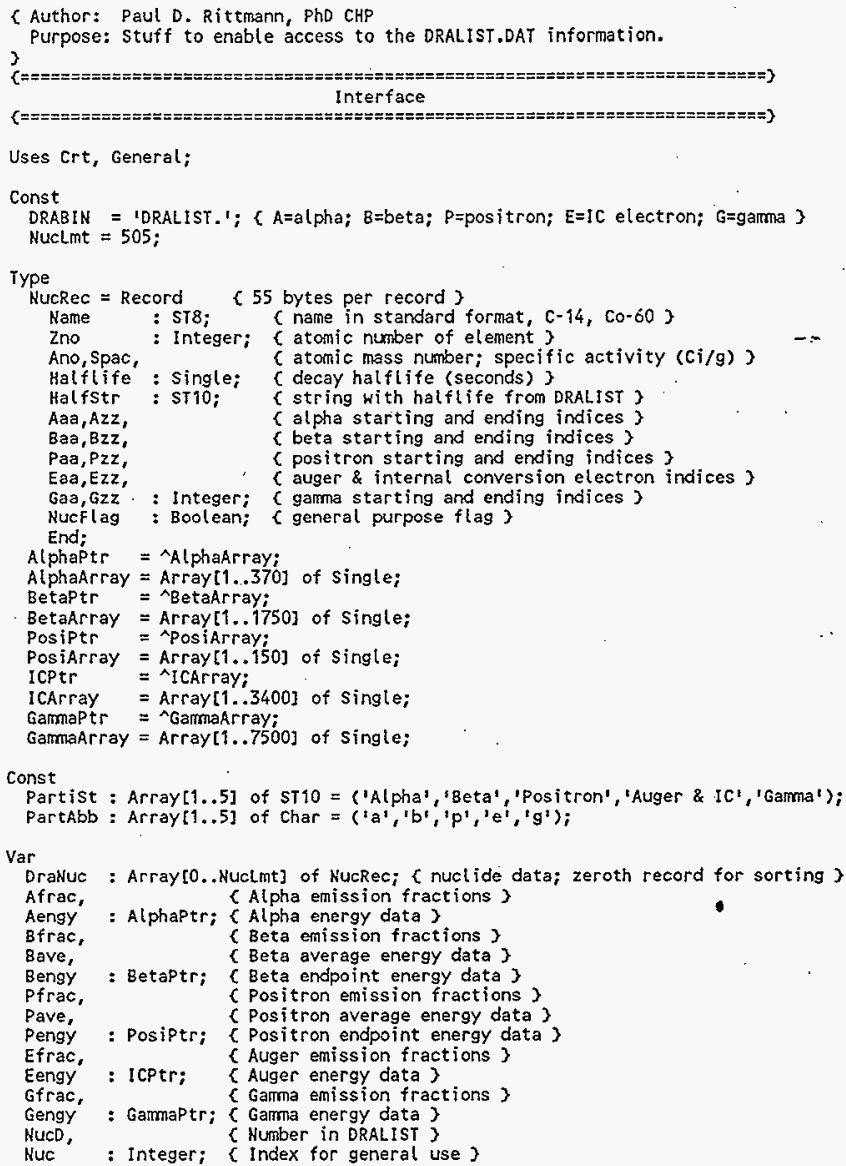

Procedure Get DRALIST $(A, B, P, I C, G$ :8yte);

(Creates and initializes the requested arrays. For example, Get_DRALIST $(0,1,1,1,0)$ will initialize only the beta, positron and ic electron arrays for fraction and energy. The average energy arrays are only initialized if the number is $>1$. 3

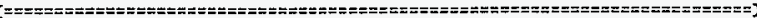
Implementation

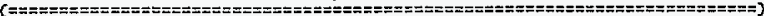

Procedure Get DRALIST(A,B,P, IC, G:Byte);

6 Creates and initializes the requested arrays.

For example, Get DRALIST $(0,1,1,1,0)$ will initialize only the beta, positron and IC electron arrays for fraction and energy. The average energy arrays are only initialized if the number is $>1$. 


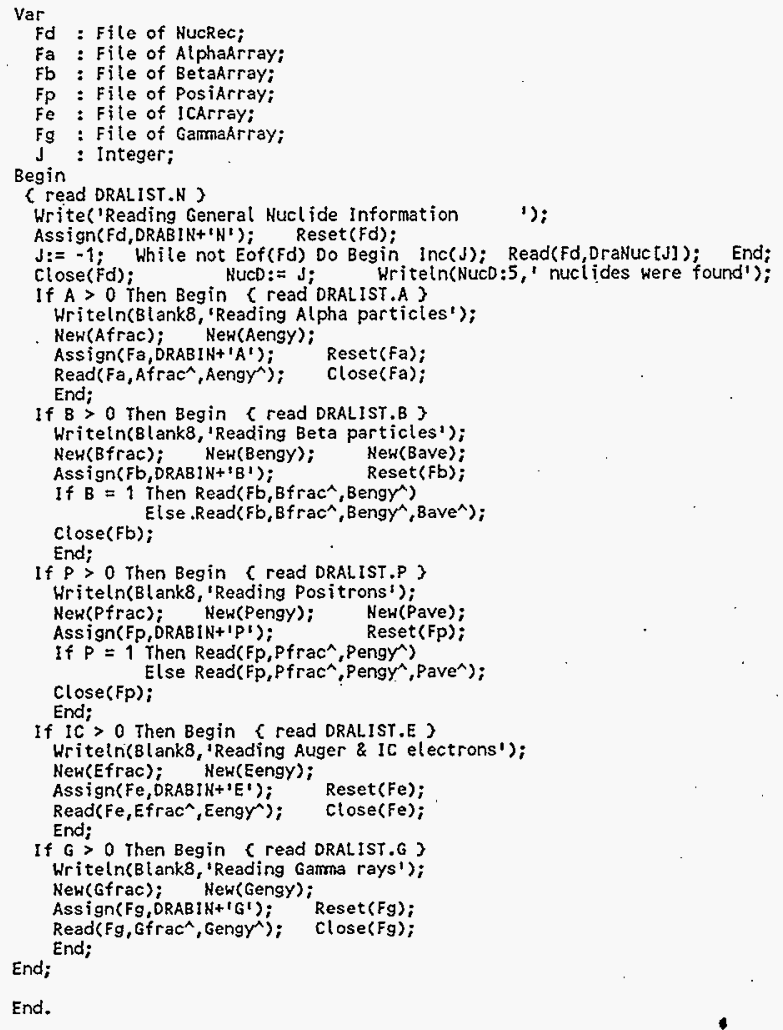




\section{PROGRAM LISTING - SRCFRAC.PAS}

Unit Srcfrac;

( Author: Paul D. Rittmann, PhD CHP

Purpose: Stuff to calculate the fraction of alphas, betas, positrons, and Auger/IC electrons enitted from a unit concentration in the source.

Usage: Calling program must call init fermi to initialize variables and open Fermifile for reading. Calling program should end by closing Fermifile.

了

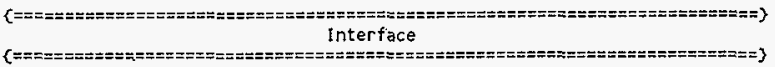

Uses Crt, General;

Const

Fermifile = 'FERMI.BIN'; ( binary fite with Fermi function values )

al pha $=137.03599 ;$ \{ fine structure constant
$m c 2=510.9991 ; \quad$ rest mass energy of the electron, Kev

SourcDflt $=2000 ;\left(\mathrm{mg} / \mathrm{cm}^{2}\right.$ assumed source thickness $\}$

ThickDflt $=5 ;\left\{\mathrm{mg} / \mathrm{cm}^{2}\right.$ assumed shielding thickness $\}$

Geomblt $=0.5$; ( assumed efficiency for detection geometry )

SpcN1 $=500$; number of points at which the spectrum is computed

SPCN = SpCN1-1; ( constant in more frequent use than SpeN1 ?

REpt $=35 ; \quad$ number of beta range-energy conversion data points )

RApt $=15 ; \quad$ (number of alpha range-energy conversion data points ?

SrCNO = 200; ( number of thicknesses needed for source integration \}

$N \operatorname{tanZ}=100 ; \quad$ (number of elements)

Mump $=420 ; \quad$ ( number of electron momenta in the (ibrary)

keVmin $=9.34 ;$ (minimun acceptable beta particle energy)

Sreunit $=1 \mathrm{mg} / \mathrm{cm}^{2}:$;

Type

REarray = Array [1. REpt $]$ of single: $\{$ electron range-energy from RHH $p .123$ \}

RAarray = Array[1..RApt] of Singte; (Alpha range-energy from RHH p.125\}

Betaspec = Array[1...SpcN1] of Doubí:

RangeArray $=$ Array $[0 .$. Srcko $]$ of Double;

DoubleArray $=$ Array $[1$...NumP $]$ of Double;

Const

Symbol : Array[1..Numz,1..2] of Char =

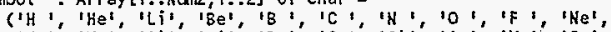

'Nat, 'Mgt, 'Al', 'Si!', IP I', IS ', 'Cl', 'Art, 'K ', 'Cat,

'SC', 'Ti', 'V ', 'Cr', 'Mnt, 'Fe', 'Co', 'Nit, 'Cus', 'Zn',

'Ga', IGe', 'AS', 'Sel, 'Br', IKr', 'Rb', 'Sr', 'Y', IZr',

'Nb', 'MO', 'TC', 'Ru', IRh', 'Pd', 'Ag', 'Cd', 'In', 'Sn',

'Sb', 'Te', 'I ', 'Xe', 'Cs', 'Ba', 'La', 'Ce', 'Pr', 'Nd',

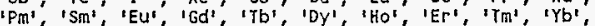

'Lu', 'HF', 'Ta', 'W', 'Re', 'OS', 'I I', 'Pt', 'AU', 'Hg',

ITl', 'Pb', 'Bit, 'Po', 'At', 'Rn', 'Fr', 'Ra', 'AC', 'Th',

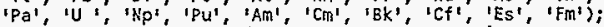

Sgnst : Árray[false., true]' of ST8 = ('Electron', 'Positron');

RangA : RAarray $=(0.647,0.957,1.293,1.668,2.108,2.599,3.155,3.788$,

$\left(\mathrm{mg} / \mathrm{cm}^{2} \mathrm{j}\right.$ $4.487,5.198,5.974,6.801,7.693,8.573 ; 9.478 ;$

EngYA : RAarray $=(1000,1500,2000,2500,3000,3500,4000,4500$,

( $\mathrm{keV}$ ) 5000,$5500 ; 6000,6500 ; 7000,7500,8000$; ;

RangB : REarray $=(1,1,5,2,2.5,3,4, \quad 5,6,8,10,15,20$, $\left(\mathrm{mg} / \mathrm{cm}^{2}\right)$ (25, 30, 40, 50, $60,80^{\prime}, 100,150,200^{\prime}, 250,300,400$, $500,600,800,1000,1500,2000,2500,3000,4000,5000,6000) ;$

EngyB : REarray $=(24.5,30.5,35.5,40,44,51,57,63,73,83,104,124$, \{ kev $\}$ : $143,161,194,225,255,310,363,485,595,700,800,1000$, $1190,1370,1740,2100,3000,3940,4880,5840,7700,9500,11200)$;

Var

Farbid : Integer; ( forbiddeness, $0,1,2,3$ )

positron, (true if particle is an positron, false if electron)

Allowed, (Forbid $<2$ \}

Maybe : Boolean; \{ Forbid $=0$ or 2 \}

Emax, Emin, ( imits on beta particle kinetic energy, kev

Wmax, Whin, \{limits on total energy, $\mathrm{mc}^{2}$ units\}

Ifctr, Ideltal, (factor to calculate successive source thicknesses)

Tdel ta2,

Ealphao,

Ealphar,

EbetaR,

intecration factors used in Combine Beta Layers?

( energy of alpha which penetrates to Thicko s

( energy of alpha which penetrates to Thick0+Rmax)

( energy of beta which penetrates to Thick0+Rmax) 


\begin{tabular}{|c|c|}
\hline $\begin{array}{l}\text { DeltaE, } \\
\text { Rmax, } \\
\text { Geometry, } \\
\text { Thicko } \\
\text { Aslp } \\
\text { Bslp } \\
\text { SpecSum, } \\
\text { SpecE, } \\
\text { Specf } \\
\text { Ebeta, } \\
\text { DepthB } \\
\text { Pss, } \\
\text { Fermo } \\
\text { FF }\end{array}$ & 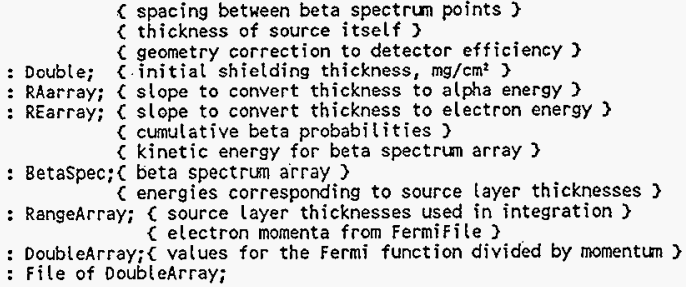 \\
\hline
\end{tabular}

Procedure Init Fermi;

(Computes the values for the source integration process.)

Procedure Beta Spectrum( $\mathbf{z}$ : integer );

( Reads Fermifile to initialize the Fermo array, computes and normalizes the beta spectrum. Uses the global variables FF, Forbid (assigns values to Allowed and Maybe), Emax (computes Himax), DeltaE, SpecE [], SpecF [] 3

Function Range MonoE( Eal: Single) : Double;

6 Computes the depth from which monoenergetic particles may be detected. The range for alpha particles uses linear interpolation; the range for IC electrons uses semi-log interpolation.

The global variable positron is used to tell them apart.

Positron must be true for alphas and false for Auger \& ic electrons. Also uses the global variables Ealpha0, Ebeta[1, and DepthB[]. Returns the value of the depth, in $\mathrm{g} / \mathrm{cm}^{2}$.)

Function Combine_Beta_Layers : Double;

6 sums the contributions from increasingly deep source layers using the trapezoid rule on exponentially increasing source thicknesses. simpsons rute is used to integrate the beta spectrum.

Uses the global variables Emax, Deltae, SpecE[], SpecF[J, Ebetall and

Depth[]. Returns the value of the sum, in $\mathrm{g} / \mathrm{cm}^{2}$. $\}$

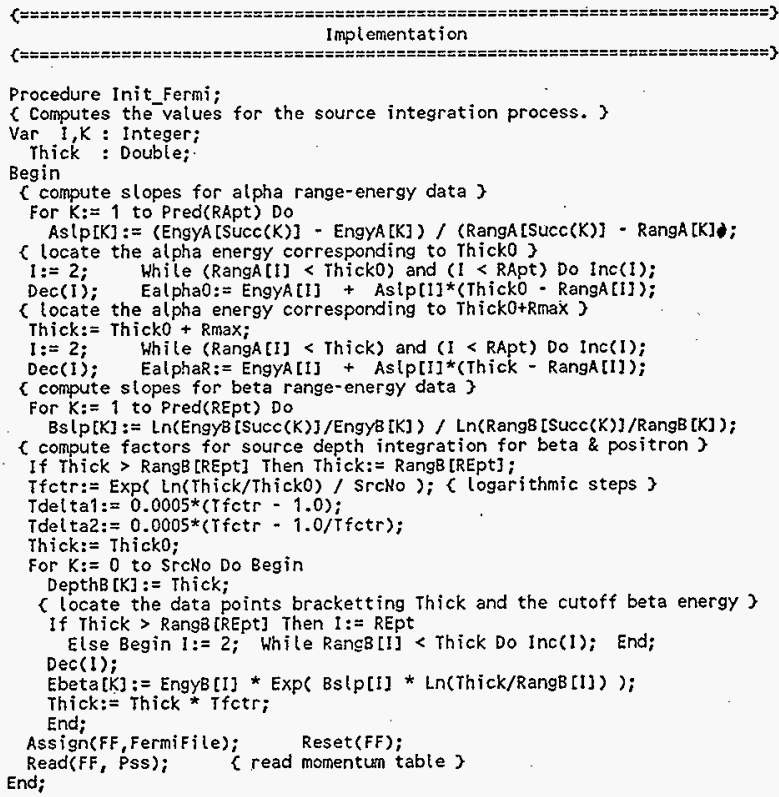



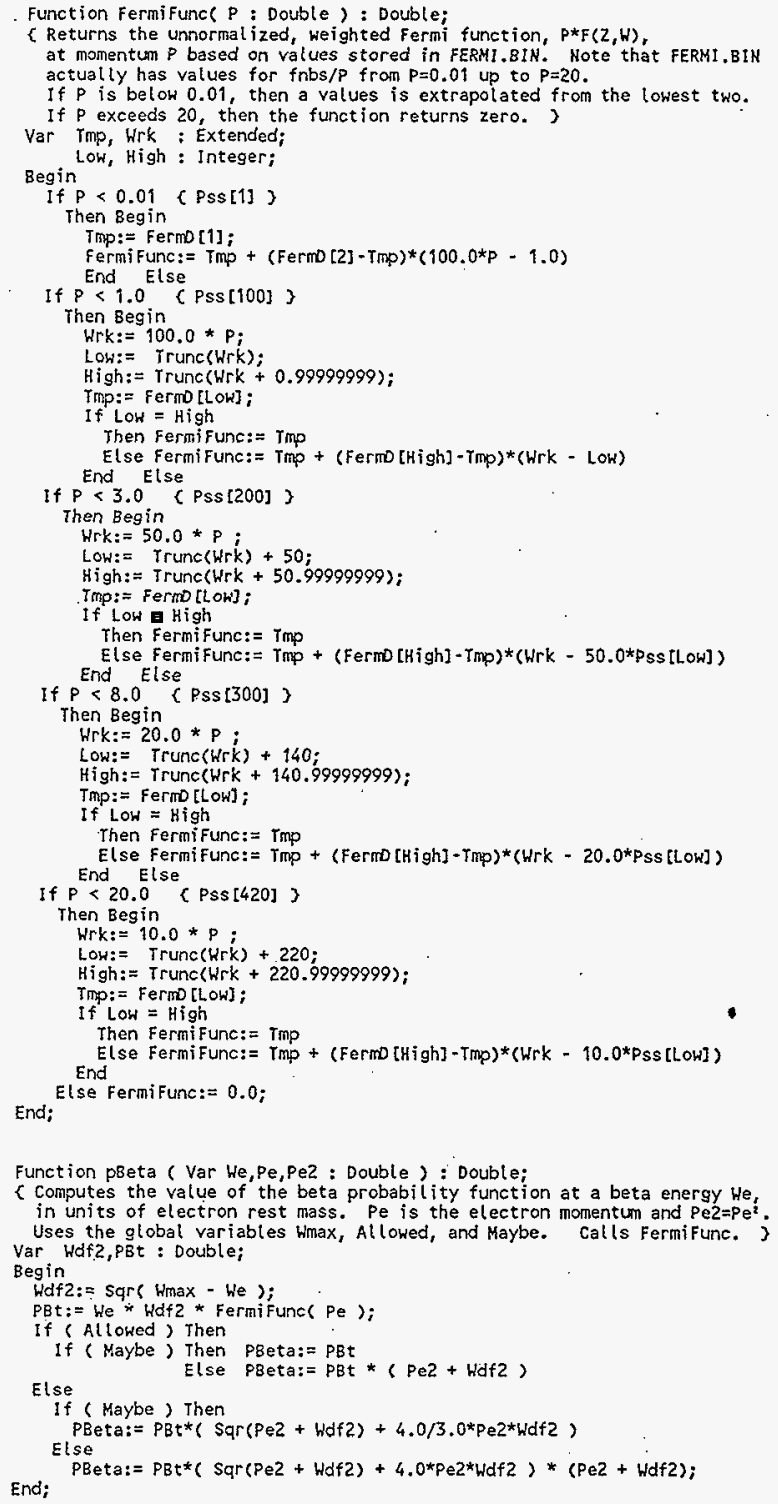
the beta spectrun. Uses the global variables FF, forbid (assigns values to Allowed and Maybe), Emax (computes Wmax), DeltaE, SpecE[], SpecF [].

$Z$ is the atonic number of the parent nuclide.

Var 1,Fermz: integer;

Wine, He, hinx2,

Pe,Pe2,PZA, Sum, Tmp : Double;

Begin

If Positron Then Fermz: $=z-1$ Etse Fermz: $=\mathbf{z}+1$;

( read Fermi function table for this atomic number)

Seek(FF, FermZ); Read(FF, Fermo):

Allowed: = Forbid $<2 ; \quad$ Maybe: $=$ not Odd(Forbid);

Wmax:= Emax/ma2 + 1.0; Hmx2:= Sqr(Wmax);

DeltaE: = Emax / SpeN1;

Winc:= Del taE / me2;

Sum: $=0.0 ; \quad P Z A:=-2.0^{\star} \mathrm{Pj}^{\star}$ FermZ/atpha;

For $I:=1$ to speN Do Begin

Pe2: $=$ Imp* $($ He +1.0$)$;

Tmp: $=$ pBeta ( We, Pe, Pe2 ):

If Positron Then Tmp: = Tmp * $\operatorname{Exp}\left(P Z A^{*} \mathrm{We} / \mathrm{Pe}\right)$;

SpecF [I] := Jmp;

If Odd(I) Then Sum: $=$ Sum $+4^{\star} T m p$ ( Simpson's Rule )

End;

Else Sum: $=$ Sum $+2 \star$ imp;

PZA:= DeltaE / 3.0; Sum: = Sum * PZA:

SPecE[SPcH1] $:=$ Emax; SpecF[SpcN1] $:=0.0$;

SpecSum[SpcN1] $:=0.0$

For $1:=$ spck downto 1 Do Begin

We:= SpecF[1]/ Sum; SpecF[l]:= Ne; ( store normalized probability $)$

If. I $=$ SpcN Then Begin

Pe:=We; Pe2: $=4$ *We;

SpecSum [SpcN] : $=0.5 *$ We*Del tak;

End Else

If $I=$ Pred(SpcN) Then Begin

Pe: $=$ Pe $+4^{*}$ We: Pe2:= Pe2 + We: (simpson's Rule ?

SpecSum[Pred $(\mathrm{SpCN})]:=$ Pe2*PZA;

End Else

If Odd(I) Then Begin

$\mathrm{Pe}:=\mathrm{Pe}+\mathrm{We} ; \quad \mathrm{Pe}:=\mathrm{Pe}+\mathrm{SpecF}[\operatorname{Succ}(1)]+4 * \mathrm{We}$; SpecSum $[1]:=\mathrm{Pe}$ PZA + SpecSum $[$ SpcN]

End

Else Begin

Pe2: $=\mathrm{Pe} 2+\mathrm{We}: \quad \mathrm{Pe}:=\mathrm{Pe}+\operatorname{SpecF}[\operatorname{Succ}(1)]+4^{*} \mathrm{We} ;$

Specsum $[1]:=$ Pe2*PZA;

End;

nd;

Function Range Monok (Eal:single) : Double;

( Computes the depth from which monoenergetic particles may be detected.

The range for alpha particles uses linear interpolation; the range fpr

Ic electrons uses semi-log interpolation.

The global variable positron is used to tell them apart.

Positron must be true for alphas and false for Auger \& ic electrons.

Also uses the global variables EalphaO, Ebeta[], and DepthB [].

Returns the value of the depth, in $\mathrm{g} / \mathrm{cm}^{2}$. )

Var

TotThick: Double; ( fraction detected, $\mathrm{g} / \mathrm{cm}^{2}$ for one particle $)$

Begin

TotThick:=0.0: I::=2:

If Positron

Then Begin

If Eal > EalphaR Then TotThick: = Thick0+Rmax Else

If Eal > Ealphao Then Begin

While (EngyA[I] < Eal) and (I < RApt) Do Inc(I)

Dec(t);

TotThick:= RangA[I] + (Eal - EngYA[I])/Aslp[I];

Else End

If Eal > Ebeta[SrcNo] Then rotThick: ThickotRmax Else

If Eal > Ebeta[0] Then Begin

While (EngYB [I] < Eal) and (I < REpt) Do Inc(I);

Dec(I);

TotThick:= RangB [I] * Exp! Ln(Eal/EngyB[I]) / Bslp[I] ); End;

TotThick:=0.001*(TotThick - Thick0):

If TotThick $<0.0$ Then TotThick: $=0.0$;

Range MonoE:= TotThick; 


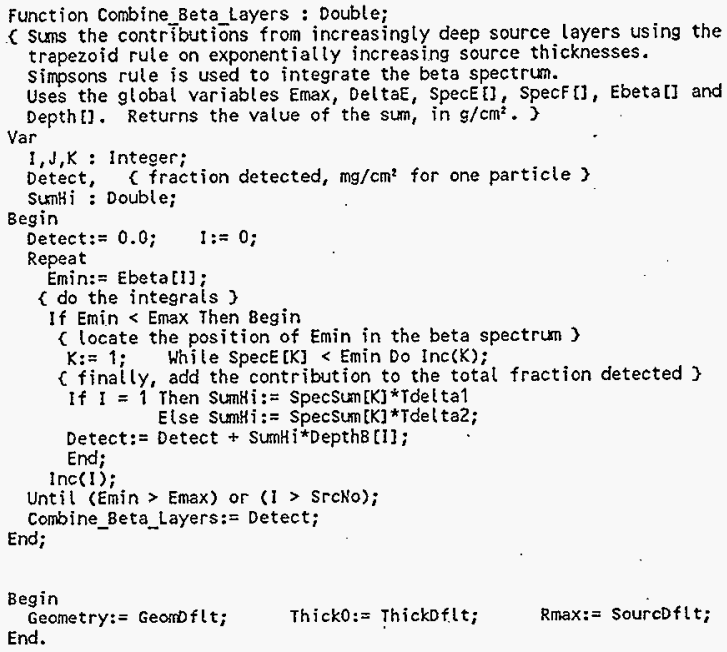




\section{PROGRAM LISTING - NUCLIST.PAS}

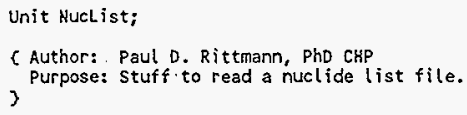

Function Valid Number(Strt,Fini:Byte; Var NewVat:Double): Boolean;

( Looks through Oneline from Strt to Fini for the first vatid number which is stored in MewVal. Returns false if an invalid number was found. Exponential numbers without the E (FORTRAN notation) are accepted. Any commas are removed. 3

Function Nuclide Block : Boolean;

6 Finds the information on one nuclide group from the nuclide list in $\mathrm{fn}$. Returns true if a block was found. Wuclide format is C-14, Co-60. Uses the global variables Onel ine and $f n$. Assigns vatues to the global variables NucN, Nuclide [], AmtVal [1 and Factor. called as part of a repeat loop, i.e., Repeat

If Nuclide Block Then 8 egin [ process info about this block ] For Nuc: $=1$ to Nuch Do Begin [ process infortnation obout this nuclide] End; Until End;

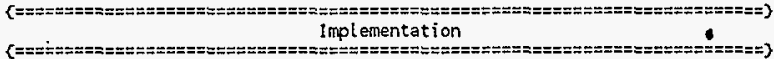

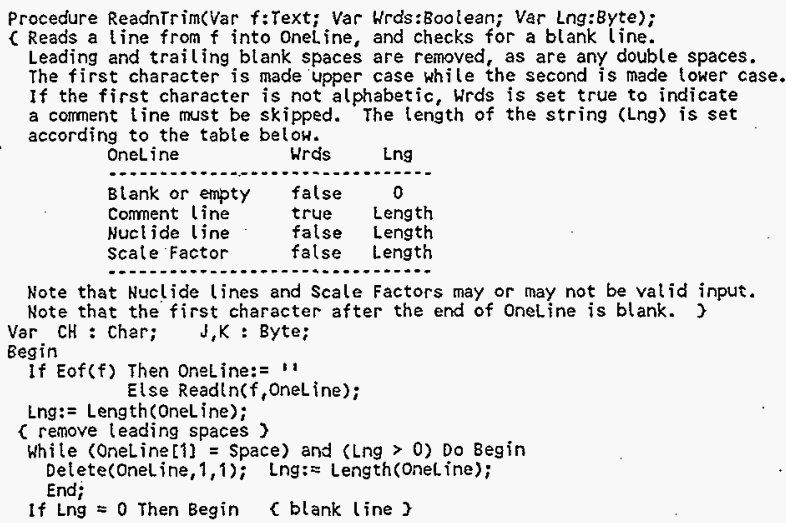

Note that Nuclide lines and Scale Factors may or may not be valid input. Note that the first character after the end of oneline is blank. J 


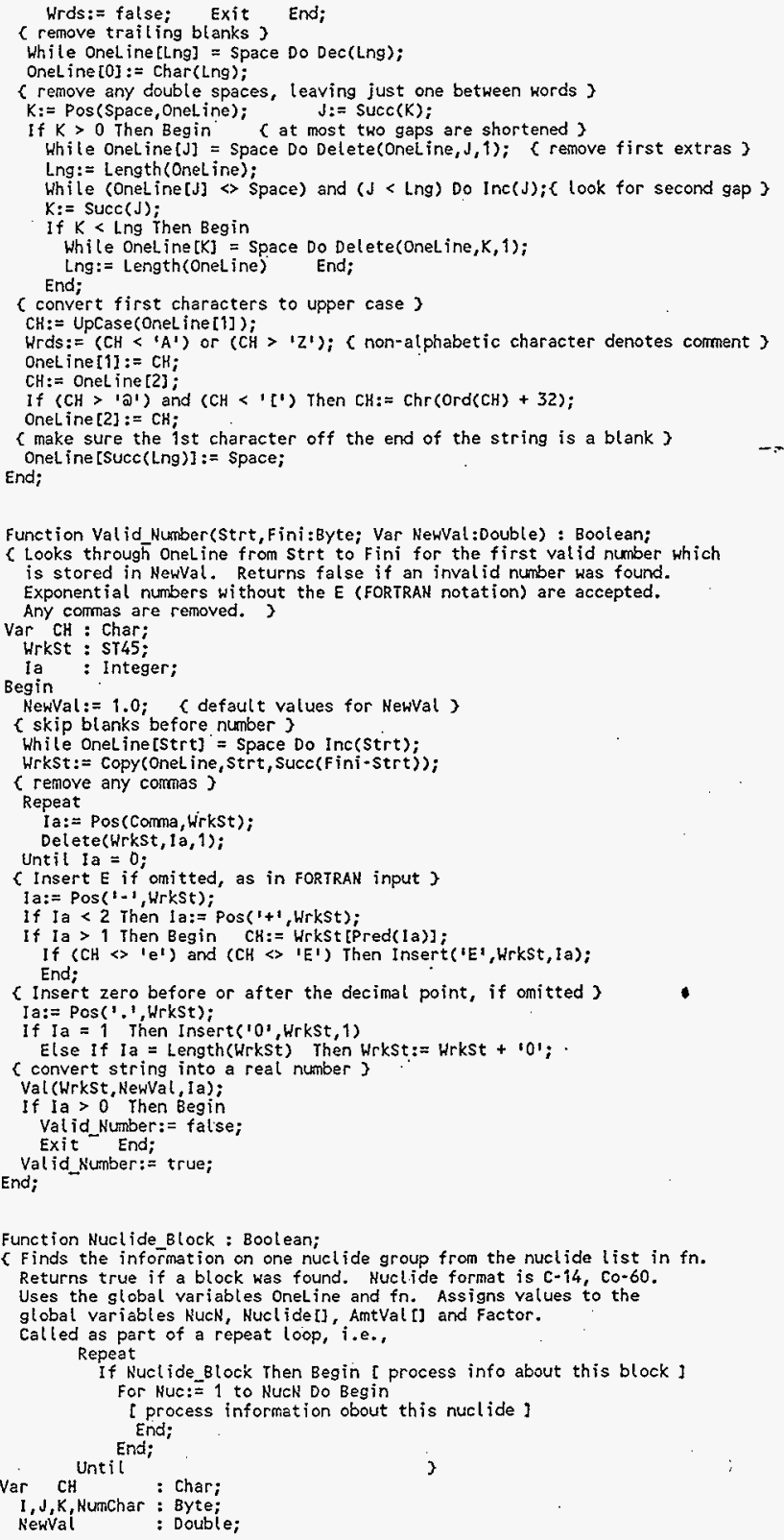


Fctrline,

Corment

: Boolean;

Begin

Nuck: $=0$;

Repeat

ReadnTrim(fn, Coment, Nunchar); Inc(L ineCount);

If (NumChar $>0$ ) and not comment then Begin

Inc(NucN);

Nuclide [Nuck] := B Lank8;

\{ store nuclide name. in standard format \}

Nucl ide [NucN, 1$]:=$ oneL ine [1];

If OneLine [2] <'a' Then Nuclide [Nuck, 2]:= Space

Else Nuct ide [Nuch, 2]:= OneL ine [2];

FctrLine:= Copy (Nuct ide [NucN], 1,2) = 'XX';

If Fctrline

Then Begin $1:=3$; $\operatorname{Dec}(\mathrm{NuCH}) ;$ End

Else Begin \{read the atomic mass number \}

$I:=2 ;\{$ skip over non-numeric characters $\}$

While (Oneline [I] < '0') or (Oneline [i] > '9') Do Inc(l);

Hucl ide [NucN, 3] := Dash;

$\mathrm{J}:=4 ;$ \{ store the numeric characters as atonic mass number

While (OneLine [1] $>1 / 1$ ) and (Onet ine [I] $<$ ':') Do Begin

Nucl ide [NuCN, J]: = onel ine III;

Inc(J); inc(I); End;

CH:= OneLine [I];

If $\left(\mathrm{CH}=\mathrm{M}^{\mathrm{M}}\right.$ ) or $(\mathrm{CH}=1 * 1)$ Then $\mathrm{CH}:=\mathrm{I}^{\prime} \mathrm{m}$;

If $\mathbf{C H}>$ Space then Begin Nuclide $[\mathrm{NuCN}, \mathrm{J}]:=\mathrm{CH}$; [nc(I); End;

If Nuclide [NuCN, 2] = Space Then Begin

- Delete(Nuclide[NucN], 2,1);

Nuctide[Huch] : = Nuclide[NucN] + Space;

End d:

If I > Numchar \{ no number on line?

Then If Fctiline Then Factor: $=1.0$

Else AmtVal $[N u C N]:=$ Factor

Else If Valid Number(i, Numchar, NewVal)

Then if Ectrline Then Factor:= NewVal

Else AmtVat [NucN]:= Factor ${ }^{\mathrm{Ne}} \mathrm{W}$ Val

Else Begin Textattr:= White;

Writeln('Invalid number in' nuclide (ist at line ', LineCount, ${ }^{1}{ }^{1}$ );

Writeln(oneline); Textattr:= Yellow;

Writeln; Writeln('Run Terminated!! Correct the nuclide file!!');

End;

Halt; End;

Until. Nunchar $=0$; $\quad$ empty line $\}$

End;

Nucl ide Block: = NueN $>0$;

End. 
Waste Management Federal Services of Hanford, Inc.

MSIN

N. A. Homan

H6-25

Eluor Daniel Northwest

P. D. Rittmann

R3-26

Lockheed Martin Services, Inc.

Central Files

$\quad$ B1-07 